\title{
Seções de Variedades Analíticas
}

\author{
JoÃo Niv́aldo TOMAZELLA \\ Orientadora: \\ Profa. Dra. Maria Aparecida Soares Ruas
}

Tese de Doutorado apresentada ao Instituto de Ciências Matemáticas e de Computação, da Universidade de São Paulo, como parte dos requisitos para obtenção do título de "Doutor em Ciências - Área: Matemática".

USP - São Carlos

Junho de 1999 
À Vera, Carolina e Lucas 


\section{Agradecimentos}

A Deus por ter me dado esta oportunidade.

À Cidinha os meus mais sinceros agradecimentos, pelo incentivo, pela dedicação, pela calma e paciência com que sempre me orientou durante o desenvolvimento deste trabalho.

À minha esposa Vera, pela sua dedicação, compreensão e apoio em todas os momentos.

Aos meus pais.

Aos professores Farid Tari e Marcelo Saia pelas valiosas conversas e a acima de tudo pela amizade.

Aos colegas da UFSCar pelo apoio e amizade.

Aos colegas de pós-graduação.

A todos os funcionários do ICMC-USP-São Carlos, em particular as "meninas" da biblioteca.

À Beth, à Laura e à Marília pelo zelo com que sempre tratam de nossos assuntos acadêmicos.

Ao Departamento de Matemática da UFSCar por ter dado condições para o desenvolvimento deste trabalho. 


\begin{abstract}
In this work we study the topological triviality of families of sections of analytic varieties. We partially extend results of B. Teissier and T. Gaffney, showing that the integral closure of the tangent space of the groups $\mathcal{R}_{V}$ and $\mathcal{K}_{R V}$ is an adequate infinitesimal object to study topological triviality and equisingularity of families of sections. The methods we introduce allow us to prove precise results on deformations by terms of positive weights of a weighted homogeneous analytic variety. Results M. Saia on the determination of the integral closure of an ideal in terms of its Newton polyhedron are used to describe an efficient method of equisingularity of Newton-non degenerate families of sections.
\end{abstract}




\section{Resumo}

O objetivo deste trabalho é estudar a trivialidade topológica de famílias de seções de variedades analíticas. Estendemos parcialmente os resultados de B. Teissier e T. Gaffney, mostrando que o fecho integral do espaço tangente dos grupos $\mathcal{R}_{V}$ e $\mathcal{K}_{R V}$ é o objeto infinitesimal que dá uma condição suficiente para a trivialidade topológica de famílias de seções. Procuramos também definir um conceito de equisingularidade para famílias de seções,

que chamaremos de $V$-equisingularidade. Os métodos utilizados permitem a obtenção de resultados precisos quando a variedade analítica é quase homogênea e a família de seções é uma deformação de um germe quasehomogêneo $f$ consistente com $V$, por termos de filtração maior ou igual a filtração de $f$. Resultados de M. Saia sobre a determinação do fecho integral de um ideal através de seu poliedro de Newton são usados para descrever um método eficiente para garantir a trivialidade topológica de famílias Newton não-degeneradas. 


\section{Índice}

Introduçāo 1

1 Preliminares 4

1.1 Germes e equivalência de germes ... . . . . . . . . . . . 4

1.2 Germes de Variedades Analíticas . . . . . . . . . . . . . 7

1.3 Condições de Whitney . . . . . . . . . . . . . . 8

2 Equivalência de Seçōes

2.1 As relações de equivalência . . . . . . . . . . . . . . . . . . 12

2.2 Condições necessárias e suficientes para

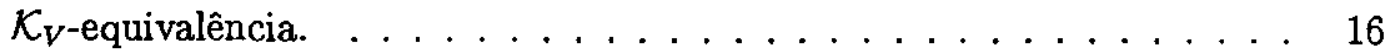

3 Determinação Finita 22

3.1 Campos de Vetores em Variedades . . . . . . . . . . . . . . . . 22

3.2 Critério Infinitesimal . . . . . . . . . . . . . . . . . 24

3.3 Critério Geométrico e Outras Propriedades . . . . . . . . . . . . . . 27

3.4 Seções das Singularidades $S_{k} \ldots \ldots \ldots$. . . . . . . . . . . 33

4 Trivialidade Topológica de Família de Seções de Hipersuperfícies 52

4.1 Fatos básicos sobre fecho integral de ideais . . . . . . . . . . . . 52

4.2 O Teorema Principal . . . . . . . . . . . . . . . . 53

4.3 O Caso Quase-Homogêneo . . . . . . . . . . . . . . . 57

4.4 O Fecho Integral e o Poliedro de Newton . . . . . . . . . . . . . 63

$5 \quad C^{0}-\mathcal{K}_{R V}$-Trivialidade Topológica $\quad 67$ 
6 Equisingularidade de Famílias de Seções de Variedades Analíticas 71 6.1 Os resultados de Teissier e Gaffney . . . . . . . . . . . . . 71

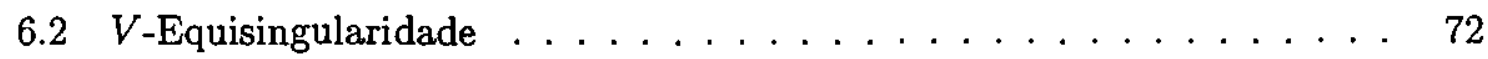

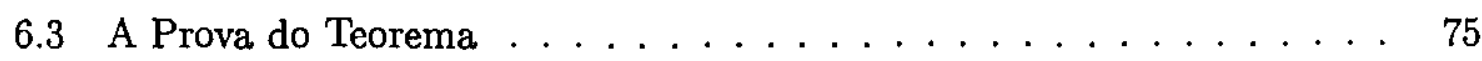

6.4 Observação final . . . . . . . . . . . . . . . . 78

$\begin{array}{ll}\text { Bibliografia } & 80\end{array}$ 


\section{Introdução}

Uma questão importante no estudo de famílias de conjuntos analíticos e de famílias de aplicações diferenciáveis com singularidades é: Quando todos os elementos da família são equivalentes (topologicamente equivalentes), ou, em outras palavras, quando a família é em algum sentido equisingular? Para este propósito em meados dos anos 60, Thom e Whitney criaram a teoria de estratificação. Uma estratificação de um conjunto é uma partição do conjunto em variedades, chamadas estratos, localmente finita, e satisfazendo a condição de fronteira: se um estrato tem parte em comum com o fecho de outro estrato então ele está inteiramente no fecho do outro estrato.

H. Whitney [45], em 1965 define as condições $A$ e $B$ de regularidade para uma estratificação e mostra que uma variedade analítica $V$ sempre admite uma estratificação que satisfaz tais condições de regularidade. Desde então, o problema de trivialidade topológica e equisingularidade de famílias de variedades analíticas ou de aplicações diferenciáveis tem sido estudados por vários autores entre os quais B. Teissier ([38], [39]), T. Gaffney ([10], [12], [13]), D.Trotman ([41], [42]), L.Wilson([46]), T.Kuo ([25]). O objetivo deste trabalho é estudar a trivialidade topológica de famílias de seções de variedades analíticas.

Seja $(V, 0) \subset\left(K^{n}, 0\right),(K=\mathbb{R}$ ou $\mathbb{C})$ um germe de variedade analítica de $\left(K^{n}, 0\right)$. Uma seção de $V$ é um conjunto $S$ obtido como a interseção de $V$ com um germe de uma variedade analítica $(X, 0) \subset\left(K^{n}, 0\right)$.

Podemos estudar as seções de $V$ de duas formas, conforme $X$ seja dada por uma parametrização $g:\left(K^{m}, 0\right) \rightarrow\left(K^{n}, 0\right)$ ou na forma implícita, ou seja, como a imagem inversa de uma aplicação $l:\left(K^{n}, 0\right) \rightarrow\left(K^{p}, 0\right), X=l^{-1}(0)$. Neste trabalho, estaremos em geral, considerando o segunda situação. Usamos o fecho integral de certos ideais ou módulos para estudar a trivialidade topológica de seções de variedades analíticas definidas implicitamente. 
Em nosso contexto, os homeomorfismos que trivializam uma dada família de seções devem preservar a variedade analítica $V$. Estaremos interessados nos grupos $\mathcal{R}_{V}, \mathcal{K}_{R V}$, $\mathcal{K}_{V}$ e $\mathcal{A}_{V}$ que denotaremos por $\mathcal{G}_{V}$, que preservam de alguma forma a variedade analítica $V$ e são subgrupos dos grupos clássicos da teoria singularidade $\mathcal{R}, \mathcal{K}$ e $\mathcal{A}$.

O objetivo central do trabalho é estender resultados de Teissier e Gaffney, mostrando que o fecho integral do espaço tangente dos grupos $\mathcal{R}_{V}$ e $\mathcal{K}_{R V}$ é o objeto infinitesimal que dá uma condição suficiente para a trivialidade topológica de famílias de seções. Procuramos também definir um conceito de equisingularidade para famílias de seções, que chamaremos de $V$-equisingularidade.

Começamos no capítulo 1 dando as noções preliminares básicas da teoria de singularidades, germes de variedades analíticas, estratificação de variedades e as condições A e B de Whitney.

No capítulo 2 definimos as relações de equivalências $\mathcal{K}_{V}, \mathcal{A}_{V}, \mathcal{R}_{V}$ e $\mathcal{K}_{R V}$ que podemos usar para classificar as seções de $V$ e mostramos que em certas condições estas equivalências se relacionam.

No capítulo 3 definimos o $\operatorname{Derlog}(V)$ de uma variedade analítica $V$, bem como suas principais propriedades. Definimos os espaços tangentes $T \mathcal{G}_{V}(h)$ às órbitas dos grupos $\mathcal{G}_{V}=\mathcal{R}_{V}, \mathcal{K}_{V}$ e $\mathcal{K}_{R V}$, e damos condições algébricas e geométricas necessárias e suficientes para a determinação finita. Finalizamos o capítulo com a classificação das seções de $S_{k}$ definidas por submersões.

O capítulo 4 contém a parte principal do trabalho. Nele, propomos condições suficientes para a trivialidade topológica de famílias de seções com métodos baseados no fecho integral do espaço tangente do grupo $\mathcal{R}_{V}$, estendendo resultados de Teissier [38] e Gaffney [10]. O resultado principal, o Teorema 4.2, estabelece que uma condição suficiente para que uma boa deformação $h:\left(K^{n} \times K, 0\right) \rightarrow(K, 0), K=\mathbb{R}, \mathbb{C}$ seja topologicamente trivial é que $\frac{\partial h}{\partial t} \in \overline{T \mathcal{R}_{V}(h)}$. Os métodos utilizados na prova deste teorema permitem a obtenção de resultados precisos quando a variedade analítica é quase homogênea e a família de seções é uma deformação de um germe quase-homogêneo $f$ consistente com $V$, por termos de filtração maior ou igual a filtração de $f$. Resultados de M. Saia [35] sobre a determinação do fecho integral de um ideal através de seu poliedro de Newton são usados para descrever um método eficiente para garantir a trivialidade topológica de famílias Newton não-degeneradas.

No capítulo 5 usamos os resultados de Gaffney [10], sobre fecho integral de módulos 
para estender parcialmente os resultados do capítulo 4 para o grupo $\mathcal{K}_{R V}$.

O objetivo do capítulo 6 é propor uma definição para equisingularidade de famílias de seções e mostrar que as condições do Teorema 4.2 implicam esta equisingularidade. 


\section{Capítulo 1}

\section{Preliminares}

\subsection{Germes e equivalência de germes}

Neste trabalho iremos estudar as propriedades locais de aplicações diferenciáveis $f$ : $K^{n} \rightarrow K^{p}$, onde $K=\mathbb{R}$ ou $\mathbb{C}$ e diferenciável significa $C^{\infty}$ ou analítico real quando $K=$ $\mathbb{R}$ e holomorfa quando $K=\mathbb{C}$. Como estamos interessados apenas no comportamento de $f$ na vizinhança de um ponto singular, trabalhamos com germes de aplicação em um ponto $a \in K$, isto é , classes de equivalência de aplicações que coincidem em alguma vizinhança de $a$. Os pontos $a$ e $f(a)$ são chamados respectivamente fonte e meta do germe. Usa-se a notação $f:\left(K^{n}, a\right) \rightarrow K^{p}$ para indicar um germe de aplicação com fonte $a$ e a notação $f:\left(K^{n}, a\right) \rightarrow\left(K^{p}, f(a)\right)$ quando queremos fixar a fonte e a meta.

Fazendo mudanças de coordenadas, podemos considerar $a=0$. Indicaremos por: $\mathcal{O}(n, p)$ o conjunto dos germes holomorfos $f:\left(\mathbb{C}^{n}, 0\right) \rightarrow \mathbb{C}^{p}, \mathcal{C}(n, p)$ o conjunto dos germes $C^{\infty}, f:\left(\mathbb{R}^{n}, 0\right) \rightarrow \mathbb{R}^{p}$ e $\mathcal{A}(n, p)$ o conjunto dos germes analíticos reais $f:\left(\mathbb{R}^{n}, 0\right) \rightarrow \mathbb{R}^{p}$.

Para $p=1$, usaremos a notação $\mathcal{O}_{n}, \mathcal{C}_{n}$ e $\mathcal{A}_{n}$, respectivamente para $\mathcal{O}(n, 1), \mathcal{C}(n, 1) \mathrm{e}$ $\mathcal{A}(n, 1)$. Estes conjuntos têm estrutura de anel local e seu ideal maximal em qualquer dos casos será indicado por $\mathcal{M}_{n}$. Este ideal é constituido pelos germes de funções $f:\left(K^{n}, 0\right) \rightarrow(K, 0)$, ou seja, funções $f$ com $f(0)=0$.

Quando não houver perigo de confusão, usaremos a notação comum $K(n, p), K_{n}$ e $K_{p}$, respectivamente, para $\mathcal{O}(n, p), \mathcal{O}_{n}$ e $\mathcal{O}_{p}$ quando $K=\mathbb{C}$, e $\mathcal{C}(n, p), \mathcal{C}_{n}$ e $\mathcal{C}_{p}$ ou $\mathcal{A}(n, p), \mathcal{A}_{n}$ e $\mathcal{A}_{p}$ quando $K=\mathbb{R}$.

Dado um germe de aplicação $f:\left(K^{n}, 0\right) \rightarrow K^{p}$ teremos $f=\left(f_{1}, \ldots, f_{p}\right)$ com $f_{i} \in K_{n}$ 
para $i=1, \ldots, p$. Então podemos identificar o espaço dos germes $K(n, p)$ como o $K_{n}$ módulo livre $K_{n} \times K_{n} \times \ldots \times K_{n}$ ( $p$ cópias).

Denotemos por $J^{k}(n, p)$ o conjunto das $p$-uplas de polinômios de grau menor ou igual a $k$ (sem termo constante) e por $j^{k} f(0)$ ou simplesmente $j^{k} f$ o $k$-jato de $f$ que é o polinômio de Taylor de $f$ em 0 (sem termo constante). Quando $p=1$, pode-se mostrar que $j^{k} f \equiv 0$ se, e somente se, $f \in \mathcal{M}_{n}^{k+1}$. Assim, podemos identificar o espaço de $k$-jatos de aplicações $f: K^{n} \rightarrow K, J^{k}(n, 1)$ com o espaço $\frac{K_{n}}{\mathcal{M}_{n}^{k+1}}$. Assim, tem-se

$$
J^{k}(n, p)=\frac{K_{n}}{\mathcal{M}_{n}^{k+1}} \times \frac{K_{n}}{\mathcal{M}_{n}^{k+1}} \times \ldots \frac{K_{n}}{\mathcal{M}_{n}^{k+1}}(p \text { cópias })
$$

Dada uma relação de equivalência $\mathcal{G}$ definida no conjunto dos germes de aplicações, dizemos que um germe $f$ é $k$ - $\mathcal{G}$-determinado se qualquer germe $g$ com $j^{k} f=j^{k} g$ é $\mathcal{G}$-equivalente a $f$. Dizemos que um germe $f$ é finitamente $\mathcal{G}$-determinado ou $\mathcal{G}$-finito se $f$ é $k$ - $\mathcal{G}$-determinado para algum $k<\infty$.

As relações de equivalências de nosso interesse são definidas por ações de subgrupos dos grupos que descreveremos a seguir.

$\mathcal{R}=$ grupo dos germes de difeomorfismos de $\left(K^{n}, 0\right) \rightarrow\left(K^{n}, 0\right)$. Um elemento $\phi \in \mathcal{R}$ é denominado uma mudança de coordenadas na fonte. A ação sobre um germe $f$ é dada pela composição à direita de $f$, isto é, $\phi . f=f \circ \phi^{-1}$. Dizemos que os germes de aplicações $f, g$ são $\mathcal{R}$-equivalentes se existir $\phi \in \mathcal{R}$ tal que $g=f \circ \phi^{-1}$.

$\mathcal{L}=$ grupo dos germes de difeomorfismos na meta $\left(K^{n}, 0\right) \rightarrow\left(K^{n}, 0\right)$. Um elemento $\varphi \in \mathcal{L}$ é denominado uma mudança de coordenadas na meta. A ação sobre um germe $f$ é dada pela composição à esquerda de $f$, isto é, $\varphi \cdot f=\varphi \circ f$. Desta forma dizemos que os germes de aplicações $f, g$ são $\mathcal{L}$-equivalentes se existir $\varphi \in \mathcal{L}$ tal que $g=\varphi \circ f$.

$\mathcal{A}=\mathcal{R} \times \mathcal{L}$ produto direto dos grupos anteriores, é o grupo das mudanças de coordenadas na fonte e na meta. Dado $(\phi, \varphi) \in \mathcal{A}$, ou seja, germes de difeomorfismos $\phi \in \mathcal{R}$ e $\varphi \in \mathcal{L}$, a ação em um elemento $f \in K(n, p)$ é dada por $(\phi, \varphi) . f=\varphi \circ f \circ \phi^{-1}$. Como antes, dizemos que dois germes $f$ e $g$ são $\mathcal{A}$-equivalentes se existir $(\phi, \varphi) \in \mathcal{A}$ tal que $g=\varphi \circ f \circ \phi^{-1}$.

$\mathcal{C}=$ grupo dos germes de difeomorfismos $(H, i d)$ com $H:\left(K^{n} \times K^{p}, 0\right) \rightarrow\left(K^{n} \times\right.$ $\left.K^{p}, 0\right)$ e $i d:\left(K^{n}, 0\right) \rightarrow\left(K^{n}, 0\right)$ germe da identidade, tais que

1. $H \circ i=i \operatorname{com} i(x)=(x, 0)$,

2. $\pi_{1} \circ H=i d \circ \pi_{1} \operatorname{com} \pi_{1}(x, y)=x$. 
Ou seja, $H(x, y)=(x, \theta(x, y)) \operatorname{com} \theta(x, 0)=0$. Dizemos que $g$ e $g^{\prime}$ são $\mathcal{C}$ equivalentes se existe $(H, i d) \in \mathcal{C}$ tal que $H(x, g(x))=\left(x, g^{\prime}(x)\right)$, ou seja, $H$ leva o gráfico de $g$ no gráfico de $g^{\prime}$, preservando as fibras $\pi_{1}^{-1}(x)$.

$\mathcal{K}=\mathcal{R} . \mathcal{C}$ (produto semi direto) $=$ conjunto dos germes de difeomorfismos $(H, h)$ com $H:\left(K^{n} \times K^{p}, 0\right) \rightarrow\left(K^{n} \times K^{p}, 0\right)$ e $h:\left(K^{n}, 0\right) \rightarrow\left(K^{n}, 0\right)$ tais que

1. $H \circ i=i \circ h \operatorname{com} i(x)=(x, 0)$,

2. $\pi_{1} \circ H=h \circ \pi_{1} \operatorname{com} \pi_{1}(x, y)=x$.

Ou seja, $H(x, y)=(h(x), \theta(x, y))$ com $\theta(x, 0)=0$. Dizemos que $g$ e $g^{\prime}$ são $\mathcal{K}$ equivalentes se existe $(H, h) \in \mathcal{K}$ tal que $H(x, g(x))=\left(h(x), g^{\prime}(h(x))\right)$, ou seja, $H$ leva o gráfico de $g$ no gráfico de $g^{\prime}$.

Passamos agora a descrever os espaços tangentes à ação destes grupos. Dado um germe diferenciável $f:\left(K^{n}, 0\right) \rightarrow K^{p}$, seja $\theta_{f}$ o conjunto dos germes de campos de vetores ao longo de $f$, isto é, o conjunto de germes $\zeta:\left(K^{n}, 0\right) \rightarrow T K^{p}$, tais que $\pi_{p} \circ \zeta=f$ onde $\pi_{p}: T K^{p} \rightarrow K^{p}$ é a projeção natural e $T K^{p}$ é o fibrado tangente de $K^{p}$. Temos que $\theta_{f}$ é um $K_{n}$-módulo livre, $\theta_{f}=K_{n}^{p}$. Denotamos por $\theta_{n}$ o conjunto dos germes de campos de vetores ao longo da identidade $i d_{n}:\left(K^{n}, 0\right) \rightarrow\left(K^{n}, 0\right)$, ou seja, os germes de campos de vetores de $K^{n}$. Observemos que $\theta_{n}$ é um $K_{n}$-módulo livre, $\theta_{n}=K_{n}^{n}$. Analogamente, define-se o $K_{p}$-módulo livre $\theta_{p}$. Associados a estes módulos temos os seguintes homomorfismos:

$$
t f: \theta_{n} \rightarrow \theta_{f}
$$

definido por $t f(\eta)=d f(\eta)$, onde $\eta \in \theta_{n}$ e $d f$ é a diferencial de $f$, e

$$
w f: \theta_{p} \rightarrow \theta_{f}
$$

definida por $w f(\xi)=\xi \circ f$.

A aplicação $w f$ é a aplicação induzida de $f^{*}: K_{p} \rightarrow K_{n}, f^{*}(g)=g \circ f$. Assim, $f^{*}\left(\mathcal{M}_{p}\right)$ é o ideal de $K_{p}$ gerado pelas componentes de $f$.

Os espaços tangentes a órbita de $f$ pela ação do grupo $\mathcal{R}, \mathcal{L}, \mathcal{A}, \mathcal{C}$ e $\mathcal{K}$ são respectivamente ( veja para maiores detalhes [43]):

$$
\begin{aligned}
& T \mathcal{R}(f)=t f\left(\mathcal{M}_{n} \theta_{n}\right) \\
& T \mathcal{L}(f)=w f\left(\mathcal{M}_{p} \theta_{p}\right)
\end{aligned}
$$




$$
\begin{gathered}
T \mathcal{A}(f)=t f\left(\mathcal{M}_{n} \theta_{n}\right)+w f\left(\mathcal{M}_{p} \theta_{p}\right), \\
T \mathcal{C}(f)=f^{*}\left(\mathcal{M}_{p}\right) \theta_{f} \mathrm{e} \\
T \mathcal{K}(f)=t f\left(\mathcal{M}_{n} \theta_{n}\right)+f^{*}\left(\mathcal{M}_{p}\right) \theta_{f}
\end{gathered}
$$

Um germe de aplicação $f$ é $k$-G-determinado se o conjunto dos germes com o mesmo $k$-jato de $f$ está contido na órbita $\mathcal{G} . f$ de $f$. Um resultado importante para caracterizar as $\mathcal{G}$-órbitas dos germes $\mathcal{G}$-determinados é o seguinte;

Lema 1.1 (Lema de Mather, [43], pag 484) Sejam G um grupo de Lie atuando diferenciavelmente em uma variedade $C^{\infty} M$. Seja $X$ uma subvariedade $C^{\infty}$ conexa de $M$. Então, $X$ está contida em uma única órbita $G$ se, e somente se,

(i) Para todo $x \in X, T_{x}(G . x) \supseteq T_{x} X$, e

(ii) $\operatorname{dim} T_{x}(G . x)$ é a mesma para todo $x \in X$.

Para a prova veja ([27], Lema 3.1).

\subsection{Germes de Variedades Analíticas}

O objeto de estudo deste trabalho são as variedades analíticas que definiremos a seguir.

Definição 1.1 Seja $U$ um aberto e conexo de $K^{n}$. Um subconjunto $V$ de $U$ é uma subvariedade analítica se para todo $x \in U$ existe uma vizinhança $U_{x}$ e funções analíticas $f_{1}, \ldots, f_{t}$ em $U_{x}$, tais que $V \cap U_{x}=\left\{y \in U_{x}: f_{1}(y)=0, \ldots, f_{t}(0)=0\right\}=\mathcal{V}\left(f_{1}, \ldots, f_{t}\right)$.

Estaremos interessados apenas em germes de variedades analíticas que descreveremos a seguir.

Definição 1.2 Sejam $X, Y$ subconjuntos de $K^{n}$. Dizemos que os conjuntos $X$ e $Y$ são equivalentes em 0 se existe uma vizinhança $U$ de 0 tal que $X \cap U=Y \cap U$. Uma classe de equivalência de conjuntos é chamada de germe de um conjunto. A classe de equivalência será denotada por $(X, 0)$.

Seja $f \in K_{n}$ um germe analítico. Denotaremos por $\mathcal{V}(f)$ a classe de equivalência dos conjuntos $\{x \in U: g(x)=0\}$, onde $g$ é um representante do germe $f$. 
Definição 1.3 Um germe de conjunto $V$ é um germe de variedade analítica se existem germes de funções analíticas $f_{1}, \ldots, f_{t}$ tais que $V=\mathcal{V}\left(f_{1}\right) \cap \ldots \cap \mathcal{V}\left(f_{t}\right)$.

Definição 1.4 Seja $V$ um germe de de variedade analítica. $O$ ideal de $V$ é definido como o conjunto $\mathcal{I}(V)=\left\{f \in K_{n}: f(x)=0\right.$ para todo $\left.x \in V\right\}$. Seja $\mathcal{F} \subset \mathcal{K}_{n}$. $O$ conjunto dos zeros de $\mathcal{F}$ é definido como sendo $\mathcal{V}(\mathcal{F})=\{x: f(x)=0$ para todo $f \in$ $\mathcal{F}\}$.

\subsection{Condições de Whitney}

Embora as estratificações de maior interesse em nosso trabalho sejam as estratificações de conjuntos analíticos, iniciamos definindo, mais geralmente, uma estratificação de um subconjunto de uma variedade diferenciável.

Definição 1.5 Uma estratificação de um subconjunto $Z$ de uma variedade diferenciável $M$ é uma partição localmente finita $\mathcal{S}$ de $Z$ por subvariedades diferenciaveis de $M$, chamadas de estratos, satisfazendo a condição de fronteira: se $X, Y \in \mathcal{S}$ e $\bar{X} \cap Y \neq \emptyset$ então $Y \subset \bar{X}$.

Duas importantes condições no estudo de estratificações são as condições $A$ e $B$ de Whitney, as quais descreveremos a seguir:

No que segue $X, Y$ são estratos e $Y \subset \vec{X}$.

Condição $A$ de Whitney: $\mathrm{O}$ par de estratos $(X, Y)$ satisfaz a condição $A$ de Whitney em um ponto $y \in Y$ se todo limite de hiperplanos tangentes a $X$ em $y$ contém o espaço tangente a $Y$ em $y$.

Condição $B$ de Whitney: $O$ par de estratos $(X, Y)$ satisfaz à condição $B$ de Whitney em um ponto $y \in Y$ se para todas as sequências $\left(x_{i}\right),\left(y_{i}\right)$ em $X$ e $Y$ respectivamente, ambas convergindo para $y$, se a sequência de espaços tangentes $\left(T_{x_{i}} X\right)$ converge para um subespaço $T$ e se a sequência de linhas secantes ligando $x_{i}$ a $y_{i}$ converge para a linha $L$, então $L \subset T$.

Definição 1.6 Uma estratificação de Whitney de um subconjunto $Z$ de uma variedede diferenciável $M$ é uma estratificação $\mathcal{S}$ de $Z$ que satisfaz as condições $A$ e $B$ de Whitney. 


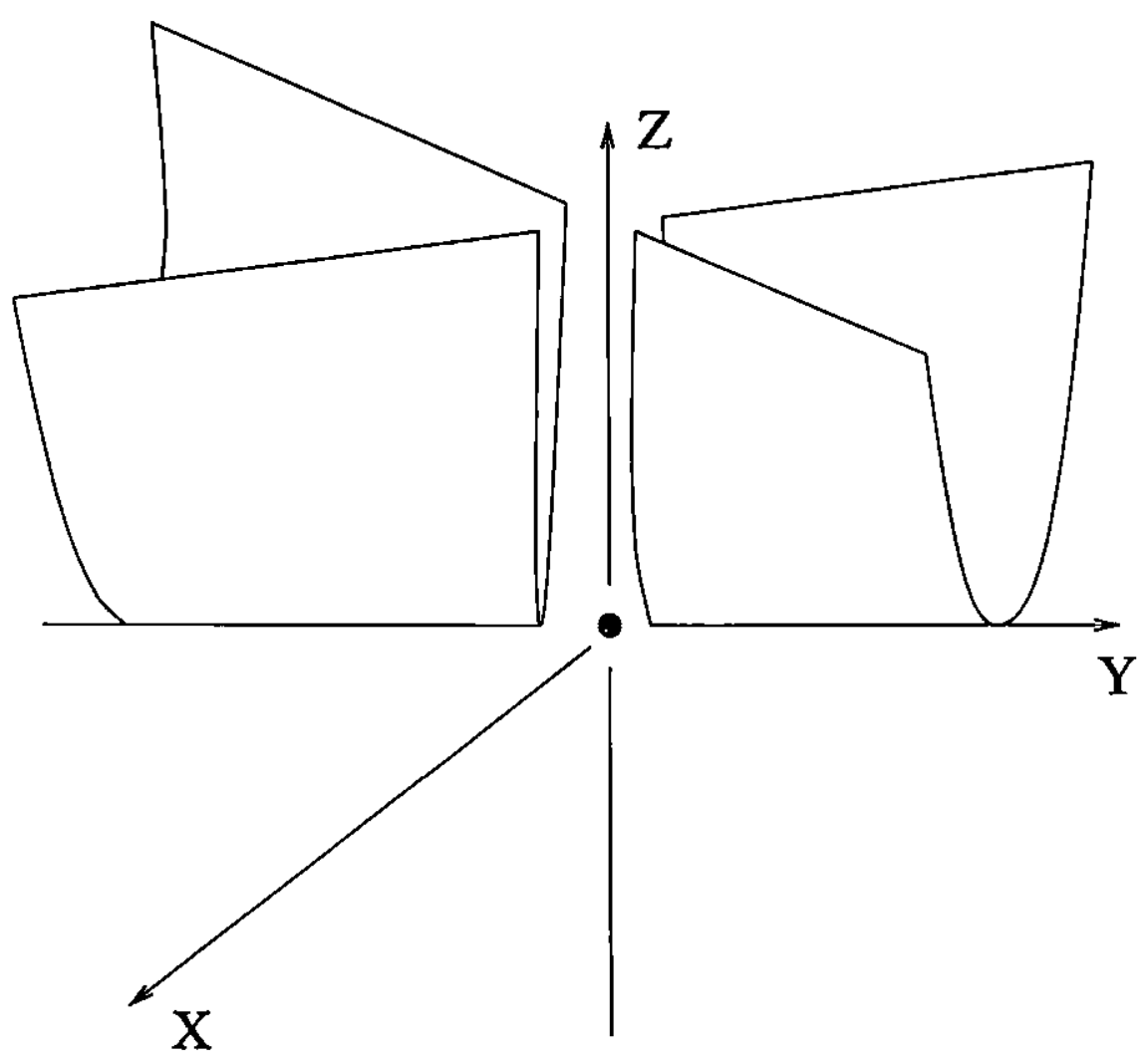

Figura 1.1: Estratificação do guarda chuva de Whitney.

Exemplo 1.1 Seja $Z \subset \mathbb{R}^{3}$ definido por $x^{2}-z y^{2}=0$, (o guarda chuva de Whitney). Uma estratificação de Whitney para $Z$ é dada pelos seguintes estratos: os complementares do eixo- $z$ em $Z$, os semi-eixos dos $z$, isto é, $z>0$ e $z<0$ e o ponto $(0,0,0)$ (ver Figura 1.1).

Uma outra condição de regularidade é a condição $W$ de Verdier, que nos diz que quando aproximamos $y$ de $X$, a distância entre o espaço tangente de $X$ em $x$ e o espaço tangente de $Y$ em $y$ vai para zero mais rápido que a distância entre $x$ e $Y$ vai para zero. No caso analítico complexo, a condição $W$ é equivalente as condições $A$ e $B$ de Whitney [41]. Para uma definição mais precisa precisamos da definição de distância entre subespaços lineares.

Definição 1.7 Sejam $U$ e $W$ subespaços lineares de um n-espaço vetorial com produto 
interno, então

$$
\operatorname{dist}(U, W)=\sup _{\substack{w \in W^{\perp}-\{0\} \\ u \in U-\{0\}}} \frac{|(w, u)|}{\|w\|\|u\|}
$$

Nas aplicações $W$ é o maior espaço e $U$ o menor espaço. Notemos que:

(a) $\operatorname{dist}(U, W)$ em geral não é igual a $\operatorname{dist}(W, U)$,

(b) $\operatorname{dist}(U, W)=0$ se, e somente se, $U \subset W$, e

(c) $\operatorname{dist}(U, W)=1$ se, e somente se, existe um vetor não nulo em $U$ ortogonal a $W$.

Definição 1.8 Suponha $Y \subset \bar{X}, X, Y$ estratos de uma estratificação de um conjunto analítico, e dist $\left(T_{y} Y, T_{x} X\right) \leq \operatorname{dist}(x, Y)^{e}$. Então $(X, Y)$ satisfaz Whitney $A$ com expoente e em $y \in Y$. A condição $W$ de Verdier é a condição Whitney $A$ com expoente 1 .

A questão fundamental no estudo de equisingularidades, é descobrir quando um conjunto $X$ algébrico ou analítico, pode ser considerado equisingular ao longo de um subconjunto não singular $Y \subset X$ em um ponto $0 \in Y$.

Definição 1.9 (Trivialidade Topológica, [38]) Seja $(X, 0) \subset\left(K^{n+1}, 0\right)$ germe de hipersuperfície analítica reduzida. Dizemos que $X$ é topologicamente trivial ao longo de $Y$ em 0 , se existe uma retração $r:\left(K^{n+1}, 0\right) \rightarrow(Y, 0)$ e um germe de homeomorfismo dos pares

$$
\left(K^{n+1}, X\right) \approx\left(r^{-1}(0) \times Y,\left(r^{-1}(0) \cap X\right) \times Y\right)
$$

compatível com $r$, isto é, tal que o diagrama abaixo comuta.

$$
\begin{array}{lll}
K^{n+1} & \approx r^{-1}(0) \times Y \\
r \searrow \quad & \swarrow p_{2} \\
& Y
\end{array}
$$

O primeiro Lema de isotopia de Thom mostra que as condições de Whitney são condições suficientes para a trivialidade topológica. 
Definição 1.10 Sejam $N$ e $P$ variedades diferenciáveis e $A$ um subconjunto de $N, \mathcal{S}$ uma estratificação de $A$ e seja $f: N \rightarrow P$ uma aplicação diferenciável. Dizemos que o conjunto $(A, \mathcal{S})$ é trivial sobre $P$ se existem um conjunto estratificado $(F, \mathcal{F})$ e um homeomorfismo $h: A \rightarrow P \times F$, com $f=\pi_{P} \circ h$, mandando $\mathcal{S}$ para a estratificação produto $P \times \mathcal{F}$.

Teorema 1.1 (Primeiro Lema de Isotopia de Thom, [18]) Sejam $N$ e $P$ variedades diferenciáveis e $A$ um subconjuto de $N, \mathcal{S}$ uma estratificação de Whitney de $A$, e seja $f: N \rightarrow P$ uma aplicação diferenciável tal que para cada estrato $X \in \mathcal{S}, f \mid X$ é uma submersão e $f \mid \bar{X} \cap A$ é própria. Então $(A, \mathcal{S})$ é localmente trivial sobre $P$. Isto é, para cada ponto $y \in P$ existe vizinhança $U$ tal que $\left(A \cap f^{-1}(U), \mathcal{S} \cap f^{-1}(U)\right)$ é trivial sobre $U$. 


\section{Capítulo 2}

\section{Equivalência de Seções}

Neste capítulo definimos as relações de equivalências $\mathcal{K}_{V}, \mathcal{A}_{V}, \mathcal{R}_{V}$ e $\mathcal{K}_{R V}$ que aparecem na classificação de seções de variedades analíticas. Propriedades destes grupos e relações entre os mesmos são apresentadas.

\subsection{As relações de equivalência}

Seja $(V, 0) \subset\left(K^{n}, 0\right),(K=\mathbb{R}$ ou $\mathbb{C})$ um germe de variedade analítica de $\left(K^{n}, 0\right)$ e $\mathcal{I} \subset \mathcal{O}_{n}$ (ou $\mathcal{A}_{n}$ ) um ideal definindo $V$, ou seja, $\mathcal{I}=\mathcal{I}(V)=\left\{\phi \in \mathcal{O}_{n}\left(\right.\right.$ ou $\left.\mathcal{A}_{n}\right): \phi(x)=$ $0 \forall x \in V\}$.

Definição 2.1 Uma seção de $V$ é um conjunto $S$ obtido como a interseç̧ão de $V$ com um germe de variedade analítica $(X, 0) \subset\left(K^{n}, 0\right)$.

Podemos estudar as seções de $V$ de duas formas, conforme $X$ seja dada por uma parametrização $g:\left(K^{m}, 0\right) \rightarrow\left(K^{n}, 0\right)$ ou como a imagem inversa, $X=l^{-1}(0), l$ : $\left(K^{n}, 0\right) \rightarrow\left(K^{p}, 0\right)$. Descreveremos a seguir estas diferentes abordagens.

No primeiro caso, isto é, tomando-se as seçōes de $V$ como imagens de germes de aplicações $g:\left(K^{m}, 0\right) \rightarrow\left(K^{n}, 0\right), m<n$, as relações de equivalências relevantes são:

Definição 2.2 Dois germes $g$ e $g^{\prime}:\left(K^{m}, 0\right) \rightarrow\left(K^{n}, 0\right)$ são $\mathcal{K}_{V}$-equivalentes (respecti-

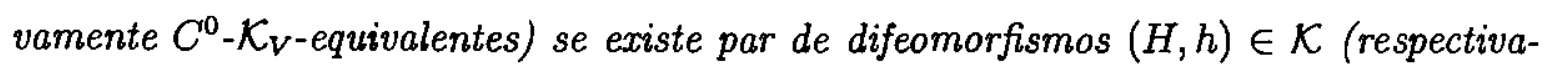
mente homeomorfismos) com $H\left(K^{n} \times V\right) \subseteq K^{n} \times V$ e $H(x, g(x))=\left(h(x), g^{\prime}(h(x))\right)$, isto é, $H$ leva o gráfico de $g$ no gráfico de $g^{\prime}$. Ou seja,

$$
\mathcal{K}_{V}=\left\{(H, h) \in \mathcal{K}: H\left(K^{n} \times V\right) \subseteq K^{n} \times V\right\}
$$


Este subgrupo de $\mathcal{K}$ pode ser visto como o produto semi-direto de $\mathcal{R}$ e $\mathcal{C}_{V}$ onde

$$
\mathcal{C}_{V}=\left\{(H, h) \in \mathcal{K}: H\left(K^{n} \times V\right) \subseteq K^{n} \times V \text { e } h \text { é germe da identidade }\right\}
$$

Exatamente como a $\mathcal{K}$-equivalência preserva a equivalência dos germes de variedades $g^{-1}(0)$, a $\mathcal{K}_{V}$-equivalência preserva a equivalência dos germes de variedades $g^{-1}(V)$. Os grupos $\mathcal{K}_{V}$ são subgrupos geométricos de $\mathcal{K}$ os quais satisfazem os teoremas básicos da teoria de singularidades, ver [6].

Definição 2.3 Dizemos que duas seções $g$ e $g^{\prime}:\left(K^{m}, 0\right) \rightarrow\left(K^{n}, 0\right)$ são $\mathcal{A}_{V}$-equivalentes (respectivamente $C^{0}-\mathcal{A}_{V}$-equivalentes) se existem germes de difeomorfismos (respectivamente homeomorfismos) $\phi:\left(K^{m}, 0\right) \rightarrow\left(K^{m}, 0\right)$ e $\varphi:\left(K^{n}, 0\right) \rightarrow\left(K^{n}, 0\right)$ tais que $g \circ \phi=\varphi \circ g^{\prime}$ e $\varphi(V)=V$.

Quando $X=l^{-1}(0)$ para um germe $l:\left(K^{n}, 0\right) \rightarrow\left(K^{p}, 0\right)$, consideramos as seguintes relações de equivalências:

Definição 2.4 Dois germes l e $l^{\prime}:\left(K^{n}, 0\right) \rightarrow\left(K^{p}, 0\right)$ são $\mathcal{R}_{V}$-equivalentes (respectivamente $C^{0}-\mathcal{R}_{V}$-equivalentes) se existe difeomorfismo (respectivamente homeomorfismo) $\phi:\left(K^{n}, 0\right) \rightarrow\left(K^{n}, 0\right) \operatorname{com} \phi(V)=V$ e $l \circ \phi=l^{\prime}$. Ou seja,

$$
\mathcal{R}_{V}=\{\phi \in \mathcal{R}: \phi(V)=V\}
$$

Quando $p=1$, este grupo tem sido estudado por vários autores entre os quais [4], [8] e [30].

Definição 2.5 Dois germes $l$ e $l^{\prime}:\left(K^{n}, 0\right) \rightarrow\left(K^{p}, 0\right)$ são $\mathcal{K}_{R V}$-equivalentes (respectivamente $C^{0}-\mathcal{K}_{R V}$-equivalentes) se existe par de difeomorfismos $(H, \phi) \in \mathcal{K}$ (respectivamente homeomorfismos) com $\phi(V)=V$ e $H(x, l(x))=\left(\phi(x), l^{\prime}(\phi(x))\right)$, isto é, $H$ leva o gráfico de l no gráfico de $l^{\prime}$. Ou seja,

$$
\mathcal{K}_{R V}=\{(H, \phi) \in \mathcal{K}: \phi(V)=V\}
$$

Este subgrupo de $\mathcal{K}$ pode ser visto como o produto semi-direto de $\mathcal{R}_{V}$ e $\mathcal{C}$. Alguns resultados sobre o grupo $\mathcal{K}_{R V}$ foram obtidos por R. Atique em [1]. 
Definição 2.6 Seja $f:\left(K^{m}, 0\right) \rightarrow\left(K^{n}, 0\right)$ germe de aplicação analítica, denotemos por $\mathcal{I}_{f}$ o ideal gerado pelas componentes $f_{1}, \ldots, f_{n}$ de $f$. Um germe $l:\left(K^{n}, 0\right) \rightarrow\left(K^{p}, 0\right)$ é uma equação de definição reduzida para $f$ se o ideal das funções que anulam sobre $\left(f\left(K^{m}\right), 0\right)$ é gerado por $\mathcal{I}_{l}$, ou seja, $\mathcal{I}\left(f\left(K^{m}\right)\right)=<l_{1}, \ldots, l_{p}>$ como um $K_{n}$-módulo.

Em ([17], pag 149) mostra-se que $f$ e $g:\left(K^{n}, 0\right) \rightarrow\left(K^{p}, 0\right)$ são $\mathcal{K}$-equivalentes se, e somente se, existe germe de difeomorfismo $h:\left(K^{n}, 0\right) \rightarrow\left(K^{n}, 0\right)$ aplicando $\mathcal{I}_{f}$ em $\mathcal{I}_{g}$, isto é, $h^{*}\left(\mathcal{I}_{f}\right)=\mathcal{I}_{g}$, onde $h^{*}: K_{n} \rightarrow K_{n}$ é o isomorfismo de anel induzido por $h$. Disto segue que se $f$ e $g$ são $\mathcal{K}$-equivalentes então existe germe de difeomorfismo $h:\left(K^{n}, 0\right) \rightarrow\left(K^{n}, 0\right)$ tal que $h\left(f^{-1}(0)\right)=g^{-1}(0)$. Observemos que não vale a recíproca, por exemplo considere $f(x)=x$ e $g(x)=x^{2}$, mas vale o seguinte.

Lema 2.1 Sejam $f$ e $g:\left(\mathbb{C}^{m}, 0\right) \rightarrow\left(\mathbb{C}^{n}, 0\right)$ com $\mathcal{I}_{f}$ e $\mathcal{I}_{g}$ ideais radicais. Se existir germe de difeomorfismo $h:\left(\mathbb{C}^{m}, 0\right) \rightarrow\left(\mathbb{C}^{m}, 0\right)$ tal que $h\left(f^{-1}(0)\right)=g^{-1}(0)$, então $f$ e $g$ são $\mathcal{K}$-equivalentes.

Prova: Basta mostrar que $h^{*}\left(\mathcal{I}_{g}\right)=\mathcal{I}_{f}$. Seja $l \in \mathcal{I}_{g}$ então $l(z)=0 \forall z \in g^{-1}(0)$. Tomando-se $x \in f^{-1}(0)$ segue-se por hipótese que $h(x) \in g^{-1}(0)$. Assim,

$$
h^{*}(l)(x)=(l \circ h)(x)=l(h(x))=0 \Rightarrow l \circ h \in \mathcal{I}\left(\mathcal{V}\left(\mathcal{I}_{f}\right)\right)=\sqrt{\mathcal{I}_{f}}=\mathcal{I}_{f}
$$

onde a penúltima igualdade vem do Teorema de Zeros de Hilbert, para germes de funçōes analíticas [20], logo $h^{*}\left(\mathcal{I}_{g}\right) \subseteq \mathcal{I}_{f}$. Como $h^{*}$ é um isomorfismo mostra-se analogamente que $\left(h^{*}\right)^{-1}\left(\mathcal{I}_{f}\right) \subseteq \mathcal{I}_{g}$, então $\mathcal{I}_{f} \subseteq h^{*}\left(\mathcal{I}_{g}\right)$. Portanto $h^{*}\left(\mathcal{I}_{g}\right)=\mathcal{I}_{f}$ e segue o resultado.

Relacionaremos a seguir a $\mathcal{A}_{V}$ classificação de seções com a $\mathcal{K}_{R V}$ classificação das equaçōes reduzidas das seções.

Proposição 2.1 Sejam $g, g^{\prime}:\left(\mathbb{C}^{m}, 0\right) \rightarrow\left(\mathbb{C}^{n}, 0\right)$ e $l, l^{\prime}:\left(\mathbb{C}^{n}, 0\right) \rightarrow\left(\mathbb{C}^{p}, 0\right)$ equações de definição para $g$ e $g^{\prime}$ respectivamente. Então:

(a) Se $g$ e g' são $\mathcal{A}_{V}$ - equivalentes então l e $l^{\prime}$ são $\mathcal{K}_{R V}$ - equivalentes.

(b) Se $g$ e $g^{\prime}$ são $\mathcal{A}$-finitamente determinados com $n>2$ el e l' são $\mathcal{K}_{R V}$ - equivalentes então $g$ e $g^{\prime}$ são $\mathcal{A}_{V}$ - equivalentes. 
Prova: (a) Se $g$ e $g^{\prime}$ são $\mathcal{A}_{V^{-}}$equivalentes então existem difeomorfismos $\phi$ : $\left(\mathbb{C}^{m}, 0\right) \rightarrow\left(\mathbb{C}^{m}, 0\right)$ e $\varphi:\left(\mathbb{C}^{n}, 0\right) \rightarrow\left(\mathbb{C}^{n}, 0\right)$ tais que $g \circ \phi=\varphi \circ g^{\prime}$ e $\varphi(V)=V$ Assim, $\varphi\left(\operatorname{Im}\left(g^{\prime}\right)\right)=\operatorname{Im}(g) \mathrm{e}$

$$
\begin{gathered}
(l \circ \varphi)^{-1}(0)=\varphi^{-1}\left(l^{-1}(0)\right) \supseteq \varphi^{-1}(\operatorname{Im}(g))=\operatorname{Im}\left(g^{\prime}\right), \text { então } \\
\mathcal{I}\left((l \circ \varphi)^{-1}(0)\right) \subseteq \mathcal{I}_{l^{\prime}} \Rightarrow(l \circ \varphi)^{-1}(0) \supseteq l^{-1}(0)
\end{gathered}
$$

também temos

$$
\begin{gathered}
\left(l^{\prime} \circ \varphi^{-1}\right)^{-1}(0)=\varphi\left(l^{-1}(0)\right) \supseteq \varphi\left(\operatorname{Im}\left(g^{\prime}\right)\right)=\operatorname{Im}(g), \text { então } \\
\mathcal{I}\left(\left(l^{\prime} \circ \varphi^{-1}\right)^{-1}(0)\right) \subseteq \mathcal{I}_{l} \Rightarrow \varphi\left(l^{\prime-1}(0)\right) \supseteq l^{-1}(0) \Rightarrow l^{\prime-1}(0) \supseteq(l \circ \varphi)^{-1}(0)
\end{gathered}
$$

Portanto

$$
(l \circ \varphi)^{-1}(0)=l^{-1}(0)
$$

como $\mathcal{I}_{l}$ e $\mathcal{I}_{l^{\prime}}$ são ideais radicais segue-se do Lema 2.1 que $l$ e $l^{\prime}$ são $\mathcal{K}_{R V}$ - equivalentes, já que $\varphi(V)=V$.

(b) Se $l$ e $l^{\prime}$ são $\mathcal{K}_{R V}$ equivalentes então existe difeomorfismo $h:\left(K^{n}, 0\right) \rightarrow\left(K^{n}, 0\right)$, $h(V)=V$ tal que $(l \circ h)^{-1}(0)=l^{-1}(0)$.

Assim, $\left(h^{-1} \circ g\right)\left(K^{m}\right)=g^{\prime}\left(K^{m}\right)$, e pela unicidade da normalização ([15]) segue-se que existe difeomorfismo $\alpha:\left(K^{m}, 0\right) \rightarrow\left(K^{m}, 0\right)$ tal que $h^{-1} \circ g=g^{\prime} \circ \alpha$, ou seja, $g$ e $g^{\prime}$ são $\mathcal{A}_{V}$ - equivalentes.

Observemos que a parte (a) da proposição acima também é verdadeira para a $C^{0}$ equivalência.

Com a hipótese adicional que $g$ e $g^{\prime}$ são germes de imersões então podemos relacionar a $\mathcal{A}_{V^{-}}$equivalência com a $\mathcal{K}_{V^{-}}$equivalência.

Proposição 2.2 Sejam $g$ e $g^{\prime}:\left(K^{m}, 0\right) \rightarrow\left(K^{n}, 0\right)$ germes de imersão. Então $g$ e $g^{\prime}$ são $\mathcal{A}_{V}$ - equivalentes se, e somente se, $g$ e $g^{\prime}$ são $\mathcal{K}_{V}$ - equivalentes.

Prova: Podemos supor $g(x)=(x, \varphi(x))$ e $g^{\prime}(x)=\left(\phi_{1}(x), \phi_{2}(x)\right)$ (fazendo mudanças de coordenadas apenas na fonte). 
Suponhamos que $g$ e $g^{\prime}$ são $\mathcal{K}_{V^{-}}$equivalentes, então existe difeomorfismo

$$
H:\left(K^{m} \times K^{n}, 0\right) \rightarrow\left(K^{m} \times K^{n}, 0\right)
$$

dado por $H(x, y)=(h(x), \theta(x, y)) \operatorname{com} \theta(x, 0)=0, \theta(x, V) \subseteq V \mathrm{e}$

$$
H(x, x, \varphi(x))=(h(x), \theta(x, x, \varphi(x)))=\left(h(x), \phi_{1}(h(x)), \phi_{2}(h(x))\right)
$$

Seja $\tilde{\theta}:\left(K^{n}, 0\right) \rightarrow\left(K^{n}, 0\right) \operatorname{com} \tilde{\theta}(y)=\theta\left(y_{1}, \ldots, y_{m}, y\right)$.

Então

$$
\begin{aligned}
\tilde{\theta} \circ g(x) & =\tilde{\theta}(x, \phi(x))=\theta(x, x, \phi(x)) \\
& =\left(\phi_{1}(h(x)), \phi_{2}(h(x))\right)=g^{\prime} \circ h(x)
\end{aligned}
$$

e $\tilde{\theta}(V) \subseteq V$, além disso, $\tilde{\theta}$ é difeomorfismo, pois $H$ o é.

Portanto, $g$ e $g^{\prime}$ são $\mathcal{A}_{V^{-}}$equivalentes.

A outra implicação é imediata já que $\mathcal{A}_{V}$ é um subgrupo de $\mathcal{K}_{V}$.

O exemplo seguinte mostra que se $g$ ou $g^{\prime}$ não é imersão então não vale a proposição acima.

Exemplo 2.1 Sejam $g, g^{\prime}:(K, 0) \rightarrow\left(K^{2}, 0\right)$ dadas por

$$
g(t)=\left(t^{2}, 0\right), g^{\prime}(t)=\left(t^{2}, t^{3}\right)
$$

$e V=\{(0, y), y \in K\}$. Tomemos $H(t, x, y)=(t, x, y-t x), H(t, V) \subseteq K \times V e$

$$
H\left(t, t^{2}, t^{3}\right)=\left(t, t^{2}, 0\right)
$$

Portanto $g$ e $g^{\prime}$ são $\mathcal{K}_{V^{-}}$equivalentes, mas $g$ e $g^{\prime}$ não são $\mathcal{A}_{V^{-}}$equivalentes, pois $g$ e $g^{\prime}$ não são $\mathcal{A}$ - equivalentes, uma vez que $g^{\prime}$ é 3 -A-determinado e $g$ não é $\mathcal{A}$-finitamente determinado.

\subsection{Condições necessárias e suficientes para $\mathcal{K}_{V}$-equivalência.}

Vamos agora dar condiçōes necessárias e suficiente para a $\mathcal{K}_{V^{-}}$equivalência. Para tanto precisamos de alguns resultados preliminares. 
Lema 2.2 Se $\mathcal{I}(V)=<\phi_{i}>_{i=1, \ldots, r}$ como um $K_{n}$-módulo então $\mathcal{I}\left(K^{m} \times V\right)=<\phi_{i}>_{i=1, \ldots, r}$ como um $K_{m+n}-$ módulo.

Prova: $P \in \mathcal{I}\left(K^{m} \times V\right) \Leftrightarrow P(x, y)=0, \forall(x, y) \in K^{m} \times V$.

Pelo lema de Hadamard ([17], pag. 100)

$$
P(x, y)-P(0, y)=\sum_{i=1}^{m} q_{i}(x, y) x_{i}
$$

onde

$$
q_{i}(x, y)=\int_{0}^{1} \frac{\partial P}{\partial x_{i}}\left(t x_{1}, \ldots, t x_{m}, y_{1}, \ldots, y_{n}\right) d t
$$

como $\left.P\right|_{K^{m} \times\{y\}} \equiv 0$ então $\left.q_{i}\right|_{K^{m} \times\{y\}} \equiv 0 \forall y \in V$

Logo, $q_{i}\left(K^{m} \times V\right) \equiv 0$, ou seja, $q_{i} \in \mathcal{I}\left(K^{m} \times V\right)$. Por hipótese temos

$$
P(0, y)=\sum_{i=1}^{r} h_{i}(y) \phi_{i}(y), \text { com } h_{i} \in K_{n}
$$

Assim,

$$
P(x, y)=\sum_{i=1}^{r} h_{i}(y) \phi_{i}(y)+\sum_{i=1}^{m} q_{i}(x, y) x_{i}
$$

então temos

$$
\mathcal{I}\left(K^{m} \times V\right) \subseteq \mathcal{I}(V)_{K_{n}}+\mathcal{I}\left(K^{m} \times V\right)<x_{1}, \ldots, x_{m}>
$$

segue-se que

$$
\mathcal{I}\left(K^{m} \times V\right) \subseteq \mathcal{I}(V)_{K_{m+n}}+\mathcal{I}\left(K^{m} \times V\right)<x_{1}, \ldots, x_{m}, y_{1}, \ldots, y_{n}>
$$

Portanto, pelo Lema de Nakayama ( [17] pag. 102), temos

$$
\mathcal{I}\left(K^{m} \times V\right) \subseteq \mathcal{I}(V)_{K_{m+n}}=<\phi_{i}>_{i=1, \ldots, r} \text { como } K_{m+n} \text {-módulo }
$$

Como a outra inclusão é imediata, o resultado segue.

Proposição $2.3 \mathrm{~g}, g^{\prime}:\left(K^{m}, 0\right) \rightarrow\left(K^{n}, 0\right)$ são $\mathcal{K}_{V}$ - equivalentes se, e somente se, existe germe de difeomorfismo $h:\left(K^{m}, 0\right) \rightarrow\left(K^{m}, 0\right)$ tal que $g^{\prime} \circ h$ e g são $\mathcal{C}_{V^{-}}$ equivalentes. 
Prova: Suponhamos que $g$ e $g^{\prime}$ sejam $\mathcal{K}_{V^{*}}$ equivalentes, então existem germes de difeomorfismos $H$ e $h$ com $H(x, y)=(h(x), \theta(x, y)), \theta(x, 0)=0, \theta(x, V) \subseteq V \forall x \in$ $K^{m}$ e $H \circ(i d, g)=\left(h, g^{\prime} \circ h\right)$.

Seja $H^{\prime}=\left(h^{-1}, \pi_{2}\right)$, onde $\pi_{2}(x, y)=y$, assim, $H^{\prime} \circ H(i d, g)=(i d, \theta(i d, g))=$ $\left(i d, g^{\prime} \circ h\right)$, ou seja, $g$ e $g^{\prime} \circ h$ são $\mathcal{C}_{V}$-equivalentes.

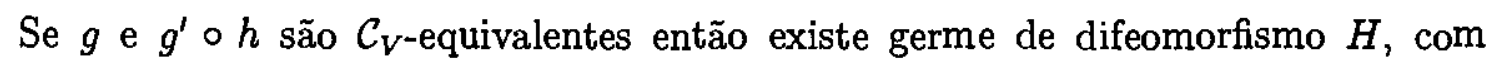
$H(x, y)=(x, \theta(x, y)), \theta(x, V) \subseteq V \forall x \in V$ e $H(i d, g)=\left(i d, g^{\prime} \circ h\right)$.

Seja $H^{\prime}(x, y)=(h(x), y)$ assim, $H^{\prime} \circ H(x, y)=(h(x), \theta(x, y))$. Portanto $H^{\prime} \circ H(i d, g)=(h, \theta(i d, g))=\left(h, g^{\prime} \circ h\right)$, ou seja, $g$ e $g^{\prime}$ são $\mathcal{K}_{V^{*}}$ equivalentes. $\diamond$

Proposição 2.4 Sejam g e $g^{\prime}:\left(K^{m}, 0\right) \rightarrow\left(K^{n}, 0\right)$ e $\mathcal{I}(V)$ ideal gerado por $\phi_{1}, \ldots, \phi_{r}$ como um $K_{n}$-módulo, então:

(a) Se $g$ e $g^{\prime}$ são $\mathcal{C}_{V}$-equivalentes então $\left\langle\phi_{i} \circ g\right\rangle_{i=1, \ldots, r}=\left\langle\phi_{i} \circ g^{\prime}\right\rangle_{i=1, \ldots, r}$.

(b) Se $g$ e $g^{\prime}$ são $\mathcal{K}_{V}$-equivalentes então $\left\langle\phi_{i} \circ g>_{i=1, \ldots, r}\right.$ e $<\phi_{i} \circ g^{\prime}>_{i=1, \ldots, r}$ são isomorfos e portanto os germes $F=\left(\phi_{1} \circ g, \ldots, \phi_{r} \circ g\right)$ e $G=\left(\phi_{1} \circ g^{\prime}, \ldots, \phi_{r} \circ g^{\prime}\right) s \tilde{a} o$ $\mathcal{K}$-equivalentes.

Prova: Parte (a), basta mostrarmos que $\left\langle\phi_{i} \circ g^{\prime}\right\rangle \subseteq\left\langle\phi_{i} \circ g\right\rangle$, pois a outra inclusão se mostra analogamente. Para tanto mostraremos que cada $\phi_{i} \circ g^{\prime}$ pode ser escrito da forma

$$
\phi_{i} \circ g^{\prime}=\sum_{j=1}^{r} a_{i j}\left(\phi_{j} \circ g\right) \operatorname{com} a_{i j} \in K_{m+n}
$$

Desde que $g$ e $g^{\prime}$ são $\mathcal{C}_{V}$-equivalentes, existe um germe de difeomorfismo $H$ tal que

$$
\begin{gathered}
H(x, y)=(x, \theta(x, y)), \theta(x, 0)=0, \theta(x, V) \subseteq V \mathrm{e} \\
H(x, g(x))=(x, \theta(x, g(x)))=\left(x, g^{\prime}(x)\right)
\end{gathered}
$$

Como $\theta(x, V) \subseteq V \Rightarrow \phi_{i}(\theta(x, V)) \equiv 0 \Rightarrow \phi_{i} \circ \theta \in \mathcal{I}\left(K^{m} \times V\right)$.

Assim, usando o Lema 2.2, podemos escrever cada $\phi_{i} \circ \theta$ da forma

$$
\phi_{i} \circ \theta(x, y)=\sum_{j=1}^{r} a_{i j}(x, y) \phi_{j}(y) \operatorname{com} a_{i j} \in K_{m+n}
$$

donde

$$
\phi_{i} \circ \theta(x, g(x))=\sum_{j=1}^{r} a_{i j}(x, g(x)) \phi_{j}(g(x))
$$


como $\phi_{i} \circ \theta(x, g(x))=\phi_{i} \circ g^{\prime}(x)$, segue-se que

$$
\phi_{i} \circ g^{\prime}=\sum_{j=1}^{r} a_{i j}\left(\phi_{j} \circ g\right)
$$

Parte $(b)$, se $g$ e $g^{\prime}$ são $\mathcal{K}_{V}$-equivalentes então existe um difeomorfismo $h$ de $K^{m}$ tal que $g^{\prime} \circ h$ e $g$ são $\mathcal{C}_{V}$-equivalentes e pela parte $\left.(a)<\phi_{i} \circ g\right\rangle=<\phi_{i} \circ g^{\prime} \circ h>$. Assim o isomorfismo $h^{*}: K_{m} \rightarrow K_{m}$ aplica $\left\langle\phi_{i} \circ g^{\prime}>\right.$ sobre $\left\langle\phi_{i} \circ g>\right.$ e portanto estes ideais são isomorfos.

O exemplo abaixo mostra que a condição $<\phi_{i} \circ g^{\prime}>$ isomorfo a $<\phi_{i} \circ g>$ não é suficiente para que $g$ e $g^{\prime}$ sejam $\mathcal{K}_{V \text {-equivalentes. }}$

Exemplo 2.2 Seja $V=\{(x, 0), x \in K\} \subseteq K^{2}, \mathcal{I}(V)=<y>_{\mathrm{O}_{2}}=<\phi>_{\mathrm{O}_{2}}$

Tomemos $g, g^{\prime}:(K, 0) \rightarrow\left(K^{2}, 0\right)$ com $g(t)=\left(t, t^{2}\right), g^{\prime}(t)=\left(0, t^{2}\right)$ temos que $(\phi \circ g)(t)=t^{2}=\left(\phi \circ g^{\prime}\right)(t)$, portanto $\langle\phi \circ g\rangle=<\phi \circ g^{\prime}>$.

Mas $g$ e $g^{\prime}$ não são $\mathcal{K}_{V}$-equivalentes, pois $g$ e $g^{\prime}$ não são $\mathcal{K}$-equivalentes.

Mas vale o seguinte

Proposiçāo 2.5 Sejam $V=\phi^{-1}(0), \phi:\left(K^{n}, 0\right) \rightarrow(K, 0)$ submersão e $g, g^{\prime}$ : $\left(K^{m}, 0\right) \rightarrow\left(K^{n}, 0\right)$ mergulhos e $h:\left(K^{n}, 0\right) \rightarrow\left(K^{n}, 0\right)$ um difeomorfismo tal que $\left.h^{*}\left(<\phi \circ g^{\prime}\right\rangle\right)=<\phi \circ g>$ então $g$ e $g^{\prime}$ são $\mathcal{K}_{V}$-equivalentes.

\section{Prova:[9]}

Passamos agora para $(V, 0) \subset\left(K^{n}, 0\right)$ na fonte.

Proposiçāo $2.6 l, l^{\prime}:\left(K^{n}, 0\right) \rightarrow\left(K^{r}, 0\right)$ são $\mathcal{K}_{R V}$ equivalentes $\operatorname{com}(V, 0) \subset\left(K^{n}, 0\right)$ se, e somente se, existe germe de difeomorfismo $\phi:\left(K^{n}, 0\right) \rightarrow\left(K^{n}, 0\right) \operatorname{com} \phi(V)=V$ tal que $l^{\prime} \circ \phi$ e l são $\mathcal{C}$-equivalentes.

Prova: Suponhamos que $l$ e $l^{\prime}$ sejam $\mathcal{K}_{R V}$ - equivalentes, então existem germes de difeomorfismos $H$ e $\phi \operatorname{com} H(x, y)=(\phi(x), \theta(x, y)), \theta(x, 0)=0, \phi(V)=V \mathrm{e}$ $H \circ(i d, l)=\left(\phi, l^{\prime} \circ \phi\right)$.

Seja $H^{\prime}=\left(\phi^{-1}, \pi_{2}\right)$, onde $\pi_{2}(x, y)=y$, assim, $H^{\prime} \circ H(i d, l)=(i d, \theta(i d, l))=$ $\left(i d, l^{\prime} \circ \phi\right)$, ou seja, $l$ e $l^{\prime} \circ \phi$ são $\mathcal{C}$-equivalentes e $\phi(V)=V$. 
Se $l$ e $l^{\prime} \circ \phi$ são $\mathcal{C}$-equivalentes e $\phi(V)=V$ então existe germe de difeomorfismo $H$, com $H(x, y)=(x, \theta(x, y))$, e $H(i d, l)=\left(i d, l^{\prime} \circ \phi\right)$.

Seja $H^{\prime}(x, y)=(\phi(x), y)$ assim, $H^{\prime} \circ H(x, y)=(\phi(x), \theta(x, y))$. Portanto $H^{\prime} \circ H(i d, l)=(\phi, \theta(i d, l))=\left(\phi, l^{\prime} \circ \phi\right)$, ou seja, $l$ e $l^{\prime}$ são $\mathcal{K}_{R V}$ - equivalentes. $\diamond$

Neste trabalho, dado um germe de variedade analítica $(V, 0) \subset\left(K^{n}, 0\right)$, estaremos quase sempre definindo uma seção de $V$ por uma equação $h=0$, onde $h:\left(K^{n}, 0\right) \rightarrow$ $\left(K^{p}, 0\right)$. Assim, nosso principal interesse é a ação dos grupos $\mathcal{R}_{V}$ (respectivamente $C^{0}$ $\mathcal{R}_{V}$ ) e $\mathcal{K}_{R V}$ (respectivamente $C^{0}-\mathcal{K}_{R V}$ ) no conjunto $K(n, p)$ dos germes de aplicações analíticas $h:\left(K^{n}, 0\right) \rightarrow\left(K^{p}, 0\right)$. As equivalências que apresentamos neste capítulo permitem, muitas vezes, que os resultados do trabalho possam ser estendidos para as demais relações definidas neste capítulo.

Como uma aplicação final das equivalências estudadas, veremos na proposição abaixo que $\mathcal{A}$ classificação de bigermes se reduz à $\mathcal{A}_{V}$ ou $\mathcal{K}_{R V}$ classificação de seções. Este resultado estende para quaisquer dimensões os resultados de R. Atique [1].

Um bigerme $G:\left(K^{m}, S\right) \rightarrow\left(K^{n}, 0\right), S=\left\{x_{1}, x_{2}\right\}$ e $x_{i} \in K^{m}, i=1,2$ é a classe de equivalência das aplicações que coincidem em uma vizinhaça se $S$. Não a perde de generalidade em considerar $x_{1}=x_{2}=0$ e o germe $G$ na forma

$$
G:\left\{\begin{array}{l}
f:\left(K^{m}, 0\right) \rightarrow\left(K^{n}, 0\right) \\
g:\left(K^{m}, 0\right) \rightarrow\left(K^{n}, 0\right)
\end{array}\right.
$$

(As equivalências de bigermes são definidas por difeomorfismos distintos na fonte e um único difeomorfismo na meta, ver [1] para maior detalhes).

Proposição 2.7 Considere os bigermes

$$
\begin{gathered}
G:\left\{\begin{array}{l}
f:\left(K^{m}, 0\right) \rightarrow\left(K^{n}, 0\right) \\
g:\left(K^{m}, 0\right) \rightarrow\left(K^{n}, 0\right)
\end{array}\right. \\
G^{\prime}:\left\{\begin{array}{l}
f:\left(K^{m}, 0\right) \rightarrow\left(K^{n}, 0\right) \\
g^{\prime}:\left(K^{m}, 0\right) \rightarrow\left(K^{n}, 0\right)
\end{array}\right.
\end{gathered}
$$

com $n>2$ e $f, g, g^{\prime}$ finitamente $\mathcal{A}$-determinados. Sejam l e $l^{\prime}$ equações de definição para $g\left(K^{m}\right)$ e $g^{\prime}\left(K^{m}\right)$ respectivamente então são equivalentes: 
(i) $G$ e $G^{\prime}$ são $\mathcal{A}$ - equivalentes,

(ii) $g$ e $g^{\prime}$ são $\mathcal{A}_{V}$-equivalentes,

(iii) l e l'são $\mathcal{K}_{R V}$-equivalentes,

onde $V$ é a imagem de $f$.

Dem: De fato, se $G$ e $G^{\prime}$ são $\mathcal{A}$ - equivalentes então existem difeomorfismos $\phi_{f}$, $\phi_{g}:\left(K^{m}, 0\right) \rightarrow\left(K^{m}, 0\right)$ e $\varphi:\left(K^{n}, 0\right) \rightarrow\left(K^{n}, 0\right)$ tal que $f \circ \phi_{f}=\varphi \circ f$ e $g \circ \phi_{g}=\varphi \circ g^{\prime}$.

Portanto $\varphi(\operatorname{Im}(f)) \subseteq \operatorname{Im}(f)$, segue-se que $\varphi(V) \subseteq V$ assim $g$ e $g^{\prime}$ são $\mathcal{A}_{V \text { - equiva- }}$ lentes.

Por outro lado se $g$ e $g^{\prime}$ são $\mathcal{A}_{V^{-}}$equivalentes então existem difeomorfismos $\phi_{g}$ : $\left(K^{m}, 0\right) \rightarrow\left(K^{m}, 0\right)$ e $\varphi:\left(K^{n}, 0\right) \rightarrow\left(K^{n}, 0\right)$ tal que $g \circ \phi_{g}=\varphi \circ g^{\prime}$ e $\varphi(\operatorname{Im}(f)) \subseteq \operatorname{Im}(f)$. Portanto $\left.(\varphi \circ f)\left(K^{n}\right)\right) \subseteq f\left(K^{n}\right)$, e pela unicidade da normalização ([15], [16]), existe $\phi$ tal que $f \circ \phi=\varphi \circ f$, portanto, $G$ e $G^{\prime}$ são $\mathcal{A}$ - equivalentes. As outras implicações seguem da Proposição 2.1.

Finalizamos o capítulo com um resumo das equivalências entre as órbitas das ações dos grupos definidos.

$$
g \stackrel{\mathcal{X}_{V}}{\sim} g^{\prime g, g^{\prime}} \stackrel{\text { imersão }}{\Leftrightarrow} g \stackrel{\mathcal{A}_{Y}}{\sim} g^{\prime g, g^{\prime}} \stackrel{\mathcal{A}-\text { finitos }}{\Leftrightarrow} \stackrel{\mathcal{K}_{R V}}{\sim} l^{\prime}
$$




\section{Capítulo 3}

\section{Determinação Finita}

Resultados sobre determinação finita dos grupos $\mathcal{G}_{V}$ aqui considerados $\left(\mathcal{G}_{V}=\mathcal{K}_{V}, \mathcal{R}_{V}\right.$, $\mathcal{K}_{R V}$ ) têm sido obtidos por vários autores. $O$ grupo $\mathcal{R}_{V}$ foi estudado em [4], [8] e [30]. J. Damon desenvolve os resultados básicos para o grupo $\mathcal{K}_{V}$ em [6] e [7]. O grupo $\mathcal{K}_{R V}$ tem sido menos estudado, alguns resultados podem ser encontrado em [1].

Nas seções 3.1 e 3.2 descrevemos os resultados obtidos nas referências acima. A seção 3.3 traz nossos resultados sobre o critério geométrico da $\mathcal{K}_{R V}$-determinação finita. A seção 3.4 apresenta um teorema de classificação das seções das superfícies singulares $S_{k}$. As singularidades $S_{k}$ foram classificadas por D. Mond em [28].

\subsection{Campos de Vetores em Variedades}

Os grupos de nosso interesse são grupos de difeomorfismos que preservam uma subvariedade analítica $V$. Para a construção de tais difeomorfismos a técnica básica é a integração de campos de vetores tangentes a $V$. As principais referências para o estudo desses campos de vetores são [4], [6] e [37].

Definição 3.1 ([4]) Sejam $(V, 0) \subset\left(K^{n}, 0\right)$ germe de variedade analítica e $\xi$ um germe de campo de vetores em $\left(K^{n}, 0\right)$. Então $\xi$ é logarítmico para $(V, 0)$ se, quando considerado como uma derivação $\xi: K_{n} \rightarrow K_{n}, g \mapsto \xi g$, nós temos $\xi g \in \mathcal{I}(V)$ para todo $g \in \mathcal{I}(V)$, onde $\xi g=d g(\xi)$. $O K_{n}$ - módulo de tais campos de vetores será denotado por $\Theta_{V}$ ou $\operatorname{Derlog}(V)$. Ou seja,

$$
\Theta_{V}=\left\{\xi \in \theta_{n}: \xi g \in \mathcal{I}(V), \forall g \in \mathcal{I}(V)\right\}
$$


As principais propriedades de $\Theta_{V}$ são dadas pela proposição abaixo.

Proposição 3.1 ([4])(i) $\Theta_{V}$ é o conjunto dos germes em $0 \in K^{n}$ de campos de vetores tangentes à parte regular de $V$.

(ii) $\Theta_{V}$ é finitamente gerado como $K_{n}$-módulo ( No caso analítico real ou holomorfo).

(iii) $S e \xi \in \Theta_{V}$ e $\xi(0)=0$, então o fluxo $\varphi_{t}$ gerado por $\xi$ preserva $V$.

Dem: (i) Seja $\xi$ um campo de vetores tangentes à parte regular de $V$, denotada por $V_{0}$, e seja $g \in \mathcal{I}(V)$. Como $\left.g\right|_{V_{0}} \equiv 0$ e $\xi$ é tangente a $V_{0}$, segue-se que $\xi(g) \equiv 0$ em $V_{0}$, donde em $V$ pois $V_{0}$ é denso em $V$. Portanto $\xi(g) \in \mathcal{I}(V)$, ou seja, $\xi \in \Theta_{V}$.

Suponhamos que $\xi(g) \in \mathcal{I}(V) \forall g \in \mathcal{I}(V)$. Sejam $\phi_{1}, \ldots, \phi_{r}$ geradores de $\mathcal{I}(V)$. Assim temos que $\xi\left(\phi_{i}\right) \equiv 0$ em $V$ e $T_{x} V_{0}=\cap_{i=1}^{r} \operatorname{Ker}\left(d \phi_{i}\right)_{x}$ para todo $x \in V_{0}$, assim $\xi(x) \in T_{x} V_{0}$, ou seja $\xi$ é tangente à parte regular de $V$.

(ii) $\Theta_{V}$ é um submódulo do módulo $\theta_{p}$ o qual é noetheriano pois $\mathcal{K}_{n}$ o é.

(iii) Seja $\varphi(t, x)$ o fluxo gerado pelo campo de vetores

$$
\xi(x)=\sum_{i=1}^{p} \xi_{i}(x) \frac{\partial}{\partial x_{i}},
$$

$\operatorname{issim} \frac{\partial \varphi}{\partial t}(t, x)=\xi(\varphi(t, x))$ e $\varphi(0, x)=x$.

Seja $g \in \mathcal{I}(V)$, mostremos que $g \circ \varphi(t, x)=0$ para todo $x \in V$.

$$
\begin{aligned}
\frac{\partial}{\partial t}(g \circ \varphi(t, x)) & =\sum_{i=1}^{p} \frac{\partial g}{\partial x_{i}}(\varphi(t, x)) \frac{\varphi_{i}}{\partial t}(t, x) \\
& =\sum_{i=1}^{p} \xi_{i}(\varphi(t, x)) \frac{\partial g}{\partial x_{i}}(\varphi(t, x)) \\
& =\xi(\varphi(t, x)) g(\varphi(t, x))
\end{aligned}
$$

que podemos escrever da forma

$$
\sum_{i=1}^{r} \alpha_{i}(t, x) g_{i}(\varphi(t, x)) \operatorname{com} g_{i} \in \mathcal{I}(V)
$$

donde se $x \in V,\left.\frac{\partial}{\partial t}(g \circ \varphi(t, x))\right|_{t=0}=0$. Agora prova-se por indução que $\left.\frac{\partial^{n}}{\partial t^{n}}(g \circ \varphi(t, x))\right|_{t=0}=0$ para todo $x \in V$ e $n \geq 1$, portanto $g \circ \varphi(t, x)=0$ para todo $x \in V$. 


\subsection{Critério Infinitesimal}

Indiquemos por $\mathcal{G}_{V}=\mathcal{R}_{V}, \mathcal{K}_{R V}$ ou $\mathcal{K}_{V}$

Definição 3.2 Um germe $h:\left(K^{n}, 0\right) \rightarrow\left(K^{p}, 0\right)$ é $k$ - $\mathcal{G}_{V}$-determinado se todo germe $g$, tal que $j^{k} g(0)=j^{k} h(0)$ é $\mathcal{G}_{V}$-equivalente a $h$. Se $h$ é $k$ - $\mathcal{G}_{V}$-determinado para algum $k$, dizemos que $h$ é $\mathcal{G}_{V}$-finitamente determinado.

Definição 3.3 Seja $(V, 0) \subseteq K^{n}$ um germe de variedade analítica. Uma deformação $F:\left(K^{n} \times K, 0\right) \rightarrow(K, 0)$ de um germe $f:\left(K^{n}, 0\right) \rightarrow(K, 0)$ é $\mathcal{R}_{V}$-trivial se existe familia de difeomorfismos

$$
H:\left(K^{n} \times K, 0\right) \rightarrow\left(K^{n}, 0\right)
$$

com $H(V \times K)=V, H(x, 0)=x, H(0, t)=0$ e $F(H(x, t), t)=F(x, 0)=f(x)$.

Definição 3.4 Seja $(V, 0) \subseteq K^{n}$ um germe de variedade analítica. Uma deformação $F:\left(K^{n} \times K, 0\right) \rightarrow\left(K^{p}, 0\right)$ de um germe $f:\left(K^{n}, 0\right) \rightarrow\left(K^{p}, 0\right)$ é $\mathcal{K}_{R V}$-trivial se existem familias de difeomorfismos

$$
\begin{gathered}
r:\left(K^{n} \times K, 0\right) \rightarrow\left(K^{n}, 0\right), r(x, 0)=x \text { e } r(V \times\{t\})=V \\
s:\left(K^{n} \times K^{p} \times K, 0\right) \rightarrow\left(K^{n} \times K^{p}, 0\right), s(x, y, 0)=(x, y) \text { e } \pi_{K^{p}}(s(x, 0, t))=0
\end{gathered}
$$

para $\forall t$ próximo de $t=0$ e tal que o diagrama abaixo comuta

$$
\begin{array}{crrrr}
\left(K^{n}, 0\right) & \stackrel{(x, f(x))}{\rightarrow} & \left(K^{n} \times K^{p}, 0\right) & \stackrel{\pi_{K^{n}}}{\rightarrow} & \left(K^{n}, 0\right) \\
\uparrow r(x, t) & & \uparrow s(x, y, t) & & \uparrow r(x, t) \\
\left(K^{n} \times K, 0\right) & \stackrel{(x, F(x, t), t)}{\rightarrow} & \left(K^{n} \times K^{p} \times K, 0\right) & \stackrel{\pi_{K^{n} \times K}}{\rightarrow} & \left(K^{n} \times K, 0\right)
\end{array}
$$

onde $\pi_{X}$ é a projeção canônica sobre $X$.

Definição 3.5 Seja $(V, 0) \subseteq K^{p}$ um germe de variedade analítica. Uma deformação $F:\left(K^{n} \times K, 0\right) \rightarrow\left(K^{p}, 0\right)$ de um germe $f:\left(K^{n}, 0\right) \rightarrow\left(K^{p}, 0\right)$ é $\mathcal{K}_{V}$-trivial se existem familias de difeomorfismos

$$
r:\left(K^{n} \times K, 0\right) \rightarrow\left(K^{n}, 0\right), r(x, 0)=x
$$


$s:\left(K^{n} \times K^{p} \times K, 0\right) \rightarrow\left(K^{n} \times K^{p}, 0\right), s(x, y, 0)=(x, y), \pi_{K^{p}}(s(x, 0, t))=0$

$\forall t$ e $s\left(K^{n} \times V \times K\right)=K^{n} \times V$

tais que o diagrama comuta

$$
\begin{array}{crrrr}
\left(K^{n}, 0\right) & \stackrel{(x, f(x))}{\rightarrow} & \left(K^{n} \times K^{p}, 0\right) & \stackrel{\pi_{K}}{\rightarrow} & \left(K^{n}, 0\right) \\
\uparrow r(x, t) & & \uparrow s(x, y, t) & & \uparrow r(x, t) \\
\left(K^{n} \times K, 0\right) & \stackrel{(x, F(x, t), t)}{\rightarrow} & \left(K^{n} \times K^{p} \times K, 0\right) & \stackrel{\pi_{K^{n} \times K}}{\rightarrow} & \left(K^{n} \times K, 0\right)
\end{array}
$$

O próximo resultado dá uma condição suficiente para a $\mathcal{R}_{V}$-trivialidade.

Proposição 3.2 Sejam $(V, 0) \subseteq K^{n}$ um germe de variedade analítica, $\left\{\eta_{1}, \ldots, \eta_{r}\right\}$ geradores de $\Theta_{V} e$

$$
F:\left(K^{n} \times K, 0\right) \rightarrow(K, 0)
$$

uma deformação do germe $f:\left(K^{n}, 0\right) \rightarrow(K, 0)$ com $F(0, t)=0$ para todo $t$. Se existir $\xi(x, t)=\sum_{i=1}^{r} a_{i}(x, t) \eta(x)$ com $a_{i} \in K_{n+1}, \xi(0, t)=0$ e $\frac{\partial F}{\partial t}=-\xi F$, então $a$ deformação $F$ é $\mathcal{R}_{V}$-trivial.

Dem: Seja $H(x, t)$ o fluxo gerado pelo campo de vetores $\xi(x, t)=\sum_{i=1}^{n} \xi_{i}(x, t) \frac{\partial}{\partial x_{i}}$. Assim,

$$
\frac{\partial H}{\partial t}(x, t)=\xi(H(x, t), t) \text { e } H(x, 0)=x
$$

então pela Proposição 3.1, $H$ preserva $V$. Diferenciando $F(H(x, t), t)$ com relação a $t$ obtemos

$$
\begin{aligned}
\frac{\partial}{\partial t} F(H(x, t), t) & =\sum_{i=1}^{n} \frac{\partial F}{\partial x_{i}}(H(x, t), t) \frac{\partial H_{i}}{\partial t}(x, t)+\frac{\partial F}{\partial t}(H(x, t), t) \\
& =\sum_{i=1}^{n} \frac{\partial F}{\partial x_{i}}(H(x, t), t) \xi_{i}(H(x, t), t)+\frac{\partial F}{\partial t}(H(x, t), t) \\
& =\left(\sum_{i=1}^{n} \xi_{i} \frac{\partial F}{\partial x_{i}}+\frac{\partial F}{\partial t}\right)(H(x, t), t)=\left(\xi F+\frac{\partial F}{\partial t}\right)(H(x, t), t) \equiv 0
\end{aligned}
$$

Portanto, fixando $x$, nós temos que $F(H(x, t), t)$ é constante, isto é,

$$
F(H(x, t), t)=F(H(x, 0), 0)=F(x, 0)=f(x)
$$

para todo $t$ e $x$. Para $x=0$ temos $\frac{\partial H}{\partial t}(0, t)=\xi(H(0, t), t)$ com solução única $H(0, t) \equiv 0$, pois $\xi(0)=0$. Portanto $F$ é $\mathcal{R}_{V \text {-trivial. }}$ 
Definição 3.6 Seja $f:\left(K^{n}, 0\right) \rightarrow\left(K^{p}, 0\right)$. Denotemos por $\Theta_{V}^{0}$ o subconjunto de $\Theta_{V}$ formado pelos campos de vetores que se anulam em 0 . Os espaços tangentes e os espaços tangentes estendidos às órbitas dos grupos $\mathcal{R}_{V}, \mathcal{K}_{R V}$ e $\mathcal{K}_{V}$ são respectivamente:

$$
\begin{array}{ll}
T \mathcal{R}_{V}(f)=d f\left(\Theta_{V}^{0}\right), & T \mathcal{R}_{V, e}(f)=d f\left(\Theta_{V}\right) \\
T \mathcal{K}_{R V}(f)=d f\left(\Theta_{V}^{0}\right)+f^{*}\left(\mathcal{M}_{p}\right) \theta_{p}, & T \mathcal{K}_{R V, e}(f)=d f\left(\Theta_{V}\right)+f^{*}\left(\mathcal{M}_{p}\right) \theta_{p} \\
T \mathcal{K}_{V}(f)=d f\left(\mathcal{M}_{n} \theta_{n}\right)+f^{*}\left(\Theta_{V}^{0}\right), & T \mathcal{K}_{V, e}(f)=d f\left(\theta_{n}\right)+f^{*}\left(\Theta_{V}\right)
\end{array}
$$

A próxima proposição dá uma condição necessária para a $\mathcal{G}_{V}$-determinação finita.

Proposição 3.3 Se $f:\left(K^{n}, 0\right) \rightarrow\left(K^{p}, 0\right)$ é $\mathcal{G}_{V}$-finitamente determinado então $T \mathcal{G}_{V}(f) \supset \mathcal{M}_{n}^{l} \theta_{f}$.

A prova no caso $\mathcal{K}_{V}$ pode ser encontrada em [6]. Para os grupos $\mathcal{R}_{V}$ e $\mathcal{K}_{R V}$, a parte essencial da prova, como nos grupos usuais $\mathcal{K}, \mathcal{A}, \mathcal{L}$ e $\mathcal{R}$, é notar que $J^{k} \mathcal{G}_{V}$ o conjunto dos $k$-jatos de elementos de $\mathcal{G}_{V}$ é um grupo de Lie atuando diferenciavelmente sobre $J^{k}(n, p)$. Para tanto, R. Pellikaan em [29] mostra que $J^{k} \mathcal{R}_{V}$ é um grupo de Lie e como $\mathcal{K}_{R V}=\mathcal{R}_{\nu} \cdot \mathcal{C}$ (produto semi-direto) segue que $J^{k} \mathcal{K}_{R V}$ também é um grupo de Lie.

Os teoremas sobre o critério infinitesimal para $\mathcal{G}_{V}$-determinação finita são conhecidos. Para $\mathcal{G}_{V}=\mathcal{R}_{V}$ e $\mathcal{K}_{R V}$, a demonstração pode ser encontrada em [1], [44] e para $\mathcal{K}_{V}$ em [6]. Reunimos esses resultados no teorema abaixo.

Teorema 3.1 $\operatorname{Se} T \mathcal{G}_{V}(f) \supseteq \mathcal{M}_{n}^{k} \theta_{f}$ então fé $k-\mathcal{G}_{V}$-determinado, $\mathcal{G}_{V}=\mathcal{R}_{V}, \mathcal{K}_{R V}$ e $\mathcal{K}_{V}$

O seguinte corolário é útil para a classificação, pois reduz o cálculo do espaço tangente ao caso de dimensão finita.

Corolário 3.1 $S e T \mathcal{G}_{V}(f)+\mathcal{M}_{n}^{k+1} \theta_{f} \supseteq \mathcal{M}_{n}^{k} \Theta_{p}$ então fé $k$ - $\mathcal{G}_{V}$-determinado.

Dem: Basta usar o Lema de Nakayama ( [17], pag. 102), uma vez que $T \mathcal{G}_{V}(f)$ é um $K_{n}$-módulo, para $\mathcal{G}_{V}=\mathcal{R}_{V}, \mathcal{K}_{R V}$ e $\mathcal{K}_{V}$.

Um método eficiente para classificação de singularidades finitamente determinadas é o método das Transversais Completas descrito por Bruce, Kirk e du Plessis em [3]. Antes de enunciarmos o teorema para os grupos de nosso interesse precisamos da seguinte definição: 
Definição 3.7 (a) Denotaremos por $\Theta_{V}^{1}$ o subconjunto de $\Theta_{V}$ formado pelos campos de vetores cujo 1-jato é nulo. Se $f:\left(K^{n}, 0\right) \rightarrow\left(K^{p}, 0\right)$ então

$$
\begin{aligned}
& T \mathcal{R}_{V}^{1}(f)=d f\left(\Theta_{V}^{1}\right) \\
& T \mathcal{K}_{R V}^{1}(f)=d f\left(\Theta_{V}^{1}\right)+f^{*}\left(\mathcal{M}_{p}\right) \mathcal{M}_{n} \theta_{f} \\
& T \mathcal{K}_{V}^{1}(f)=d f\left(\mathcal{M}_{n}^{2} \theta_{n}\right)+f^{*}\left(\Theta_{V}^{1}\right)
\end{aligned}
$$

(b) Denotaremos por $H^{k}(n, p)$ o subespaço vetorial de todas as aplicações $K^{n} \rightarrow K^{p}$ onde as componentes são polinômios homogêneos de grau $k$.

Teorema 3.2 ([3])Seja $h:\left(K^{n}, 0\right) \rightarrow\left(K^{p}, 0\right)$ um germe e $T$ um subespaço de $H^{k+1}(n, p)$ tal que

$$
J^{k+1}\left(T \mathcal{G}_{V}^{1} h\right)+T \supset H^{k+1}(n, p)
$$

Então para qualquer $(k+1)$-jato $j^{k+1} g$ com $j^{k} g=j^{k} h, j^{k+1} g$ está na mesma $J^{k+1} \mathcal{G}_{V^{-}}^{1}$ órbita de um $(k+1)$-jato da forma $j^{k+1} h+t$, para algum $t \in T$.

Corolário 3.2 Se T $\mathcal{G}_{V}^{1} h \supseteq \mathcal{M}_{n}^{k+1} \theta_{f}$ então $h$ é $k-\mathcal{G}_{V}$-determinado.

Na seção 3.4 aplicaremos os resultados acima para classificar os germes $\left.h: \mathbb{C}^{3}, 0\right) \rightarrow$ $(\mathbb{C}, 0)$ através da $\mathcal{R}_{V}$ e $\mathcal{K}_{R V}$-equivalência, com $V=\phi_{k}^{-1}(0)$, onde $\phi_{k}$ é equação reduzida para a imagem das singularidades de tipo $S_{k}$.

\subsection{Critério Geométrico e Outras Propriedades}

Lema 3.1 Seja $f:\left(K^{n}, 0\right) \rightarrow\left(K^{p}, 0\right),(V, 0) \subseteq\left(K^{n}, 0\right)$ e $\left\{\xi_{1}, \ldots, \xi_{r}\right\}$ geradores de $\Theta_{V}$. Então $f$ é $\mathcal{K}_{R V}$-finitamente determinado se, e somente se,

$$
J f\left(\Theta_{V}\right)+f^{*}\left(\mathcal{M}_{p}\right) \supseteq \mathcal{M}_{n}^{k}
$$

para algum $k$, onde $J f\left(\Theta_{V}\right)$ é o ideal de $K_{n}$ gerado pelos $(p \times p)$-menores da matriz $\left(\nabla f_{i}\left(\xi_{j}\right)\right)_{i=1, \ldots, p}^{j=1, \ldots, r}\left(\right.$ se $\left.p>r J f\left(\Theta_{V}\right)=0\right)$.

Dem: A demonstração é uma adaptação do resultado correspondente para o grupo $\mathcal{K}$ ([11], Lema 2.12). 
Se $f$ é $\mathcal{K}_{R V}$-finitamente determinado então pela Proposição 3.3 , existe $l$ tal que $T \mathcal{K}_{R V}(f) \supset \mathcal{M}_{n}^{l} \theta_{f}$, ou seja,

$$
d f\left(\Theta_{V}\right)+f^{*}\left(\mathcal{M}_{p}\right) \theta_{n} \supset \mathcal{M}_{n}^{l} \theta_{p}
$$

Seja $u \in \mathcal{M}_{n}^{l p}=\mathcal{M}_{n}^{k}$, digamos $u=u_{1} \ldots . u_{p}$ onde $u_{i} \in \mathcal{M}_{n}^{l}$. Como $\left(0, \ldots, 0, u_{i}, 0, \ldots, 0\right) \in d f\left(\Theta_{V}\right)+f^{*}\left(\mathcal{M}_{p}\right) \theta_{f}$ então existe $\eta_{i} \in \Theta_{V}$ tal que $d f\left(\eta_{i}\right)=$ $\left(0, \ldots, 0, u_{i}, 0, \ldots, 0\right) \bmod f^{*}\left(\mathcal{M}_{p}\right) \theta_{n} \operatorname{com} \eta_{i}=a_{1}^{i} \xi_{1}+\ldots+a_{r}^{i} \xi_{r}$ e $a_{j}^{i} \in K_{n}$

Assim,

$$
d f\left(\begin{array}{ccc}
a_{1}^{1} \xi_{1}^{1}+a_{2}^{1} \xi_{2}^{1}+\ldots+a_{r}^{1} \xi_{r}^{1} & \ldots & a_{1}^{p} \xi_{1}^{1}+a_{2}^{p} \xi_{2}^{1}+\ldots+a_{r}^{p} \xi_{r}^{1} \\
a_{1}^{1} \xi_{1}^{2}+a_{2}^{1} \xi_{2}^{2}+\ldots+a_{r}^{1} \xi_{r}^{2} & \ldots & a_{1}^{p} \xi_{1}^{2}+a_{2}^{p} \xi_{2}^{2}+\ldots+a_{r}^{p} \xi_{r}^{2} \\
\vdots & \vdots & \vdots \\
a_{1}^{1} \xi_{1}^{n}+a_{2}^{1} \xi_{2}^{n}+\ldots+a_{r}^{1} \xi_{r}^{n} & \ldots & a_{1}^{p} \xi_{1}^{n}+a_{2}^{p} \xi_{2}^{n}+\ldots+a_{r}^{p} \xi_{r}^{n}
\end{array}\right)
$$

Portanto,

$$
\begin{aligned}
d f\left(\begin{array}{cccc}
\xi_{1}^{1} & \xi_{2}^{1} & \ldots & \xi_{r}^{1} \\
\xi_{1}^{2} & \xi_{2}^{2} & \ldots & \xi_{r}^{2} \\
\vdots & \vdots & \vdots & \vdots \\
\xi_{1}^{n} & \xi_{2}^{n} & \ldots & \xi_{r}^{n}
\end{array}\right) \cdot\left(\begin{array}{cccc}
a_{1}^{1} & a_{1}^{2} & \ldots & a_{1}^{p} \\
a_{2}^{1} & a_{2}^{2} & \ldots & a_{2}^{p} \\
\vdots & \vdots & \vdots & \vdots \\
a_{r}^{1} & a_{r}^{2} & \ldots & a_{r}^{p}
\end{array}\right) \\
=\left(\begin{array}{ccccc}
u_{1} & 0 & 0 & \ldots & 0 \\
0 & u_{2} & 0 & \ldots & 0 \\
\vdots & \vdots & \vdots & \vdots & \vdots \\
0 & 0 & 0 & \ldots & u_{p}
\end{array}\right) \bmod f^{*}\left(\mathcal{M}_{p}\right) \theta_{f}
\end{aligned}
$$


Ou seja,

$$
\begin{aligned}
& \left(\begin{array}{ccc}
\nabla f_{1}\left(\xi_{1}\right) & \ldots & \nabla f_{1}\left(\xi_{r}\right) \\
\nabla f_{2}\left(\xi_{1}\right) & \ldots & \nabla f_{2}\left(\xi_{r}\right) \\
\vdots & \vdots & \vdots \\
\nabla f_{p}\left(\xi_{1}\right) & \ldots & \nabla f_{p}\left(\xi_{r}\right)
\end{array}\right) \cdot\left(\begin{array}{cccc}
a_{1}^{1} & a_{1}^{2} & \ldots & a_{1}^{p} \\
a_{2}^{1} & a_{2}^{2} & \ldots & a_{2}^{p} \\
\vdots & \vdots & \vdots & \vdots \\
a_{r}^{1} & a_{r}^{2} & \ldots & a_{r}^{p}
\end{array}\right) \\
& =\left(\begin{array}{ccccc}
u_{1} & 0 & 0 & \ldots & 0 \\
0 & u_{2} & 0 & \ldots & 0 \\
\vdots & \vdots & \vdots & \vdots & \vdots \\
0 & 0 & 0 & \ldots & u_{p}
\end{array}\right) \bmod f^{*}\left(\mathcal{M}_{p}\right) \theta_{f}
\end{aligned}
$$

tomando-se o determinante em ambos os lados, segue-se que

$$
u \in J f\left(\Theta_{V}\right)+f^{*}\left(\mathcal{M}_{p}\right)
$$

Por outro lado, se

$$
J f\left(\Theta_{V}\right)+f^{*}\left(\mathcal{M}_{p}\right) \supseteq \mathcal{M}_{n}^{k}
$$

então é suficiente mostrar que $J f\left(\Theta_{V}\right) \theta_{f} \subseteq d f\left(\Theta_{V}\right)$. Consideremos a submatriz $(p \times p)$, $M=\left(\nabla f_{i}\left(\xi_{j_{s}}\right)\right)_{i=1, \ldots, p}^{s=1, \ldots, p}$, da matriz $\left(\nabla f_{i}\left(\xi_{j}\right)\right)_{i=1, \ldots, p}^{j=1, \ldots, r}$. Seja $\left\{e_{1}, \ldots, e_{p}\right\}$ a base canônica de $K^{p}$ então $(\operatorname{det} M) e_{k} \in J f\left(\Theta_{V}\right) \theta_{f}$ e

$$
(\operatorname{det} M) e_{k}=\left(\begin{array}{ccc}
\nabla f_{1}\left(\xi_{1}\right) & \ldots & \nabla f_{1}\left(\xi_{r}\right) \\
\nabla f_{2}\left(\xi_{1}\right) & \ldots & \nabla f_{2}\left(\xi_{r}\right) \\
\vdots & \vdots & \vdots \\
\nabla f_{p}\left(\xi_{1}\right) & \ldots & \nabla f_{p}\left(\xi_{r}\right)
\end{array}\right) \cdot\left(\begin{array}{c}
0 \\
\vdots \\
\operatorname{cof}\left(\nabla f_{k}\left(\xi_{j_{1}}\right)\right) \\
\vdots \\
\operatorname{cof}\left(\nabla f_{k}\left(\xi_{j_{p}}\right)\right) \\
\vdots \\
0
\end{array}\right)
$$

onde $\operatorname{cof}\left(\nabla f_{k}\left(\xi_{j_{s}}\right)\right)$ denota o cofator do elemento $\nabla f_{i}\left(\xi_{j_{s}}\right)$ na matriz $M$. 
Assim,

$$
(\operatorname{det} M) e_{k}=d f\left(\begin{array}{cccc}
\xi_{1}^{1} & \xi_{2}^{1} & \ldots & \xi_{r}^{1} \\
\xi_{1}^{2} & \xi_{2}^{2} & \ldots & \xi_{r}^{2} \\
\vdots & \vdots & \vdots & \vdots \\
\xi_{1}^{n} & \xi_{2}^{n} & \ldots & \xi_{r}^{n}
\end{array}\right) \cdot\left(\begin{array}{c}
0 \\
\vdots \\
\operatorname{cof}\left(\nabla f_{k}\left(\xi_{j_{1}}\right)\right) \\
\vdots \\
\operatorname{cof}\left(\nabla f_{k}\left(\xi_{j_{p}}\right)\right) \\
\vdots \\
0
\end{array}\right)
$$

Então,

$$
\begin{gathered}
(\operatorname{det} M) e_{k}=d f\left(\begin{array}{c}
\xi_{j_{1}}^{1} \operatorname{cof}\left(\nabla f_{k}\left(\xi_{j_{1}}\right)\right)+\ldots+\xi_{j_{p}}^{1} \operatorname{cof}\left(\nabla f_{k}\left(\xi_{j_{p}}\right)\right) \\
\xi_{j_{1}}^{2} \operatorname{cof}\left(\nabla f_{k}\left(\xi_{j_{1}}\right)\right)+\ldots+\xi_{j_{p}}^{2} \operatorname{cof}\left(\nabla f_{k}\left(\xi_{j_{p}}\right)\right) \\
\vdots \\
\xi_{j_{1}}^{n} \operatorname{cof}\left(\nabla f_{k}\left(\xi_{j_{1}}\right)\right)+\ldots+\xi_{j_{p}}^{n} \operatorname{cof}\left(\nabla f_{k}\left(\xi_{j_{p}}\right)\right)
\end{array}\right) \\
=d f\left(\operatorname{cof}\left(\nabla f_{k}\left(\xi_{j_{1}}\right)\right) \xi_{j_{1}}+\operatorname{cof}\left(\nabla f_{k}\left(\xi_{j_{2}}\right)\right) \xi_{j_{2}}+\ldots+\operatorname{cof}\left(\nabla f_{k}\left(\xi_{j_{p}}\right)\right) \xi_{j_{p}}\right)
\end{gathered}
$$

$\operatorname{logo},(\operatorname{det} M) e_{k} \in d f\left(\Theta_{V}\right)$ e, portanto $J f\left(\Theta_{V}\right) \theta_{f} \subseteq d f\left(\Theta_{V}\right)$

A proposição seguinte dá um critério geométrico para a $\mathcal{K}_{R V}$-determinação finita. Proposição 3.4 Seja $f:\left(\mathbb{C}^{n}, 0\right) \rightarrow\left(\mathbb{C}^{p}, 0\right),(V, 0) \subseteq\left(\mathbb{C}^{n}, 0\right)$. Então $f$ é $\mathcal{K}_{R V^{-}}$ finitamente determinado se, e somente se,

$$
\mathcal{V}\left(J f\left(\Theta_{V}\right)\right) \cap f^{-1}(0) \subseteq\{0\}
$$

Dem: Pelo Lema 3.1, $f$ é $\mathcal{K}_{R V}$-finitamente determinado se, e somente se,

$$
J f\left(\Theta_{V}\right)+f^{*}\left(\mathcal{M}_{p}\right) \supseteq \mathcal{M}_{n}^{k}
$$

para algum $k$. Então,

$$
\mathcal{V}\left(J f\left(\Theta_{V}\right)+f^{*}\left(\mathcal{M}_{p}\right)\right) \subseteq \mathcal{V}\left(\mathcal{M}_{n}^{k}\right)
$$

portanto,

$$
\mathcal{V}\left(J f\left(\Theta_{V}\right)\right) \cap \mathcal{V}\left(f^{*}\left(\mathcal{M}_{p}\right)\right) \subseteq\{0\}
$$


ou seja,

$$
\mathcal{V}\left(J f\left(\Theta_{V}\right)\right) \cap f^{-1}(0) \subseteq\{0\}
$$

Por outro lado, se $\mathcal{V}\left(J f\left(\Theta_{V}\right)\right) \cap f^{-1}(0) \subseteq\{0\}$ então

$$
\mathcal{I}\left(\mathcal{V}\left(J f\left(\Theta_{V}\right)+f^{*}\left(\mathcal{M}_{p}\right)\right)\right) \supseteq \mathcal{I}(\{0\})
$$

Assim, pelo Teorema de Zeros de Hilbert, para germes de funções analíticas [20]

$$
\sqrt{J f\left(\Theta_{V}\right)+f^{*}\left(\mathcal{M}_{p}\right)} \supseteq \mathcal{M}_{n}
$$

portanto $J f\left(\Theta_{V}\right)+f^{*}\left(\mathcal{M}_{p}\right) \supseteq \mathcal{M}_{n}^{k}$ para algum $k$.

A caracterização geométrica para a $\mathcal{R}_{V}$-determinação finita é um corolário da proposição anterior.

Teorema $3.3 \operatorname{Sejam}(V, 0) \subseteq\left(\mathbb{C}^{n}, 0\right)$ um germe de variedade analitica e $h:\left(\mathbb{C}^{n}, 0\right) \rightarrow$ $(\mathbb{C}, 0)$. Indiquemos por

$$
V(h)=\left\{x \in \mathbb{C}^{n}: \xi h(x)=0 \text { para todo } \xi \in \Theta_{V}\right\}
$$

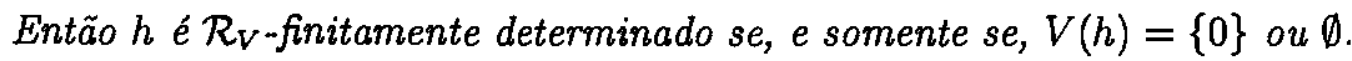

Observação 3.1 No caso real temos apenas que se $f$ é $\mathcal{G}_{V}$-finitamente determinado então: $\mathcal{V}\left(J f\left(\Theta_{V}\right)\right) \cap f^{-1}(0) \subseteq\{0\}$ para $\mathcal{G}_{V}=\mathcal{K}_{R V}$ e para $\mathcal{G}_{V}=\mathcal{R}_{V}$ o conjunto $\left\{x \in \mathbb{C}^{n}: \xi h(x)=0\right.$ para todo $\left.\xi \in \Theta_{V}\right\}$ é $\{0\}$ ou $\emptyset$.

Também temos:

Teorema 3.4 Sejam $(V, 0) \subseteq\left(K^{n}, 0\right)$ um germe de variedade analítica e $f:\left(K^{n}, 0\right) \rightarrow$ $(K, 0)$. Se $f$ é $\mathcal{R}_{V}$-finitamente determinado então $f^{-1}(0)$ é transversal a $V$ fora do 0.

Prova: Da hipótese existe $l$ tal que $d f\left(\Theta_{V}\right) \supseteq \mathcal{M}_{n}^{l}$, entāo $\mathcal{V}\left(d f\left(\Theta_{V}\right)\right) \subseteq\{0\}$. Como $\mathcal{V}\left(d f\left(\Theta_{V}\right)\right)$ descreve o conjunto dos pontos onde $f^{-1}(0)$ não é tansversal a $V$, então $f^{-1}(0)$ é transversal a $V$ fora do 0 .

Observemos que o mesmo resultado vale para $f^{-1}(c)$ para $c$ suficientemente pequeno.

O exemplo abaixo mostra que a recíproca do Teorema 3.4 não vale. 
Exemplo 3.1 Seja $V \subseteq \mathrm{C}^{2}, 0$ definida por $\varphi(x, y)=x^{3}-y^{2}=0$. Temos que $\Theta_{V}$ é gerado por $\alpha_{1}=(2 x, 3 y), \alpha_{2}=\left(2 y, 3 x^{2}\right)$. Seja $f(x, y)=y^{2}, f^{-1}(0)=$ eixo- $x$, portanto é transversal a $V$ fora do 0 mas $f$ não é $\mathcal{R}_{V}$-finito.

Definiçāo 3.8 ([6]) Seja $f:\left(\mathbb{C}^{n}, 0\right) \rightarrow\left(\mathbb{C}^{p}, 0\right),(V, 0) \subseteq\left(\mathbb{C}^{p}, 0\right) e\left\{\xi_{1}, \ldots, \xi_{r}\right\}$ geradores de $\Theta_{V}$. Dizemos que $f$ é transversal a $V$ fora do 0 se existe vizinhança $U$ de 0 tal que para $x \in\left(f^{-1}(V) \cap U\right)-\{0\}, d f(x)\left(T_{x} \mathbb{C}^{n}\right)$ é transversal ao subespaço gerado por $<\xi_{1}(f(x)), \ldots, \xi_{r}(f(x))>$, ou seja,

$$
d f(x)\left(T_{x} \mathbb{C}^{n}\right)+<\xi_{1}(f(x)), \ldots, \xi_{r}(f(x))>=T_{f(x)} \mathbb{C}^{p}
$$

Proposição 3.5 ([6],Proposição 2.2) Seja $f:\left(\mathbb{C}^{n}, 0\right) \rightarrow\left(\mathbb{C}^{p}, 0\right),(V, 0) \subseteq\left(\mathbb{C}^{p}, 0\right)$. Então $f$ é $\mathcal{K}_{V}$-finitamente determinado se, e somente se, $f$ é transversal a $V$ fora do 0 . 


\subsection{Seções das Singularidades $S_{k}$}

Nesta seção usaremos os resultados anteriores para obter a classificação das seções das singularidades $S_{k}$ definidas por submersões. A classificação é completa com exceção da singularidade $E$, para a qual apresentamos apenas uma forma pré-normal.

Teorema 3.5 Seja $V$ a imagem de $S_{k}=\left\{\left(X, Y^{2}, Y^{3}+X^{k+1} Y\right)\right\}, k \geq 1$. Um germe de submersão $h:\left(\mathbb{C}^{3}, 0\right) \rightarrow(\mathbb{C}, 0), \mathcal{R}_{V}$-finitamente determinado é $\mathcal{R}_{V \text {-equivalente a }}$ um dos seguintes germes

$$
\begin{array}{lll}
S_{k, 0} & x & \\
S_{k, 2} & y+a_{2} x^{2} & a_{2} \neq 0 \\
S_{k, 3} & y+a_{3} x^{3}+a_{4} x^{4} & a_{3} \neq 0 \\
S_{k, 4} & y+a_{4} x^{4}+a_{5} x^{5}+a_{6} x^{6} & a_{4} \neq 0 \\
\vdots & \vdots & \\
S_{k, k+1} & y+a_{k+1} x^{k+1}+a_{k+2} x^{k+2}+\cdots+a_{2 k} x^{2 k} & a_{k+1} \neq 0 \text { e } a_{k+1} \neq 1 \\
S_{k, k+1, l} & y+x^{k+1}+a_{l} x^{l}+\cdots+a_{l+k-1} x^{l+k-1} & l \geq k+2, a_{l} \neq 0 \\
S_{k, l} & y+a_{l} x^{l}+a_{l+1} x^{l+1}+\cdots+a_{l+k-1} x^{l+k-1}+\cdots & l \geq k+2, a_{l} \neq 0 \\
E_{2} & z+a_{2} x^{2} & a_{2} \neq 0 \\
E_{l}(s) & z+a_{l} x^{l}+s(x) & l \geq 3, a_{l} \neq 0, \forall k ; \text { se } k \\
& & e ́ \text { impar } e l=3(k+1) / 2, \\
& & \text { então } a_{l}^{2} \neq-4 / 27 \\
E_{l}^{1}(r) & z+b_{l-1} x^{l-1} y+r(x, y) & 2 \leq l \leq 2 k+1, b_{l-1} \neq 0, \\
& & \forall k ; \text { se } k \text { é impar } \text { e } l=(k+3) / 2, \\
& & \text { então } b_{l-1} \neq \pm 2
\end{array}
$$

Para a prova faremos uso do seguinte lema:

Lema 3.2 (i) $A$ equação

$$
\varphi(x, y, z)=2 x^{k+1} y^{2}+y^{3}-z^{2}+x^{2(k+1)} y=0
$$

é equação reduzida para a imagem de $S_{k}$.

(ii) Se $V=\varphi^{-1}(0)$ então as mudanças de coordenadas lineares $X=a x, Y=b y e$ $Z=c z \operatorname{com} b=c^{2}=a^{k+1}$ preservam $V$. 
(iii) $\Theta_{V}$ é gerado por

$$
\begin{aligned}
& \eta_{1}=(2 x, 2(k+1) y, 3(k+1) z) \\
& \eta_{2}=\left(0,2 z, x^{2(k+1)}+4 x^{k+1} y+3 y^{2}\right) \\
& \eta_{3}=\left(x^{k+1}+3 y,-2(k+1) x^{k} y, 0\right) \\
& \eta_{4}=\left(z, 0,(k+1)\left(x^{2 k+1} y+x^{k} y^{2}\right)\right)
\end{aligned}
$$

Prova: (i) A equação reduzida para $V$ é obtida usando base de Groebner.

(ii) Imediata.

(iii) Usando o programa Macaulay, observando que $\varphi$ é quase homogênea com pesos $2,2 k+2$ e $3 k+3$ para $x, y$ e $z$ respectivamente.

A técnica utilizada para a demonstração do Teorema 3.5 é o método indutivo de classificação nos espaços dos $k$-jatos $J^{k}(3,1)$. Para tanto usaremos o método das transversais completas e também os resultados sobre determinação finita descritos anteriormente e o Lema de Mather.
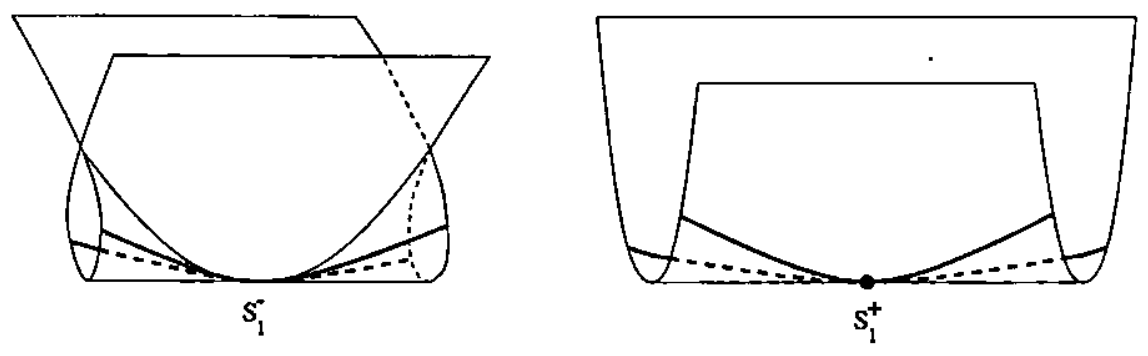

Figura 3.1: Seções de $S_{1}$ (negrito).

Passamos à prova do Teorema 3.5 .

Prova: No que segue o símbolo $\sim$ significará $\mathcal{R}_{V}$-equivalência. Para um melhor entendimento dividiremos a prova em várias etapas. Faremos a classificação para $k=$ $1,2,3$ e em seguida o caso geral.

I- Seções de $S_{1}$.

Temos neste caso que $\Theta_{V}$ é gerado por

$$
\begin{aligned}
& \eta_{1}=(2 x, 4 y, 6 z) \\
& \eta_{2}=\left(0,2 z, x^{4}+4 x^{2} y+3 y^{2}\right) \\
& \eta_{3}=\left(x^{2}+3 y,-4 x y, 0\right) \\
& \eta_{4}=\left(z, 0,2\left(x^{3} y+x y^{2}\right)\right)
\end{aligned}
$$


Classifiquemos primeiramente os 1-jatos. Tomando-se o 1-jato $h=a x+b y+c z$, temos:

$$
\begin{aligned}
& \eta_{1} h=2 a x+4 b y+6 c z \\
& \eta_{2} h=2 b z+c\left(x^{4}+4 x^{2} y+3 y^{2}\right) \\
& \eta_{3} h=a\left(x^{2}+3 y\right)-4 b x y \\
& \eta_{4} h=a z+2 c\left(x^{3} y+x y^{2}\right)
\end{aligned}
$$

Portanto $J^{\mathrm{l}}\left(T \mathcal{R}_{V}(h)\right)=<2 a x+4 b y+6 c z, 2 b z, 3 a y, a z>$. Assim, usando o Lema de Mather e mudanças de escalas temos:

Para $a \neq 0, h=a x+b y+c z \sim a x \sim x$.

Para $a=0, b \neq 0, h=b y+c z \sim b y \sim y$.

Para $a=b=0$ e $c \neq 0, h=c z \sim z$.

Portanto as órbitas em $J^{1}(3,1)$ da ação de $J^{1} \mathcal{R}_{V}$ são: $x, y, z$ e 0.

Caso (i): Classificação dos germes $h$ cujo 1-jato é $x$.

Considere o germe $h(x, y, z)=x$. Então temos

$$
\begin{array}{ll}
\eta_{1} h=2 x & \eta_{2} h=0 \\
\eta_{3} h=x^{2}+3 y & \eta_{4} h=z
\end{array}
$$

Então $T \mathcal{R}_{V}(h)+\mathcal{M}^{2} \supset \mathcal{M}^{1}$ e, portanto $x$ é 1- $\mathcal{R}_{V}$-determinado.

Caso (ii): Classificação dos germes $h$ cujo 1-jato é $y$.

Considere o germe $h(x, y, z)=y$. Então temos

$$
\begin{array}{ll}
\eta_{1} h=4 y & \eta_{2} h=2 z \\
\eta_{3} h=-4 x y & \eta_{4} h=0
\end{array}
$$

Pelo critério geométrico, $y$ não é $\mathcal{R}_{V}$-finito, uma vez que o eixo- $x$ é solução das equações $\eta_{1} h=\eta_{2} h=\eta_{3} h=\eta_{4} h=0$.

Como

$$
J^{l}\left(T \mathcal{R}_{V}^{l}(h)\right)+\mathbb{C}\left\{x^{l}\right\} \supseteq H^{l}
$$

segue do teorema da transversal completa que todos os $l$-jatos cujo $(l-1)$-jato é $y$ são $\mathcal{R}_{V}$-equivalentes a $y+a_{l} x^{l}$.

Seja $h=y+a_{l} x^{l}$. Então,

$$
\begin{array}{ll}
\eta_{1} h=2 l a_{l} x^{l}+4 y & \eta_{2} h=2 z \\
\eta_{3} h=l a_{l} x^{l+1}+3 l a_{l} x^{l-1} y-4 x y & \eta_{4} h=l a_{l} x^{l-1} z
\end{array}
$$


Determinando as soluções de $\eta_{1} h=\eta_{2} h=\eta_{3} h=\eta_{4} h=0$ e aplicando 0 critério geométrico, temos:

(a) Se $l=2, a_{2} \neq 0$ e $a_{2} \neq 1$ então $h(x, y, z)=y+a_{2} x^{2}$ é finitamente- $\mathcal{R}_{V^{-}}$ determinado.

(b) Se $l \neq 2$ e $a_{l} \neq 0$ então $h=y+a_{l} x^{l}$ é finitamente- $\mathcal{R}_{V}$-determinado.

Caso (a): Classificação dos germes cujo 2-jato é $h=y+a_{2} x^{2}, a_{2} \neq \ell$.

Quando $a_{2} \neq 1$, temos:

$$
\begin{array}{ll}
\eta_{1} h=4 a_{2} x^{2}+4 y & \eta_{2} h=2 z \\
\eta_{3} h=2 a_{2} x^{3}+6 a_{2} x y-4 x y & \eta_{4} h=2 a_{2} x z
\end{array}
$$

Então $T \mathcal{R}_{V}(h)+\mathcal{M}^{4} \supseteq \mathcal{M}^{3}$ e, portanto $y+a_{2} x^{2}$ é $3-\mathcal{R}_{V}$-determinado.

Como

$$
J^{3}\left(T \mathcal{R}_{V}^{1}(h)\right)+\mathbb{C}\left\{x^{3}\right\} \supseteq H^{3}
$$

segue do teorema da transversal completa que todos os 3-jatos cujo 2-jato é $y+a_{2} x^{2}$

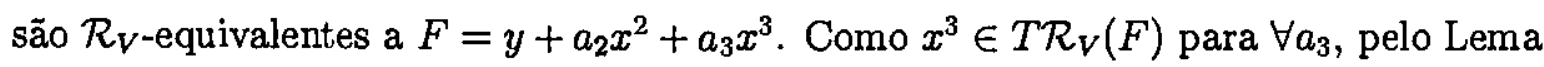
de Mather, $F$ se reduz a $y+a_{2} x^{2}$. Portanto $y+a_{2} x^{2}$ é $2-\mathcal{R}_{V}$-determinado para $a_{2} \neq 0$ e $a_{2} \neq 1$.

Quando $a_{2}=1, h=y+x^{2} \mathrm{e}$

$$
\begin{array}{ll}
\eta_{1} h=2 x^{2}+2 y & \eta_{2} h=2 z \\
\eta_{3} h=2 x^{3}+2 x y & \eta_{4} h=2 x z
\end{array}
$$

Portanto, pelo critério geométrico, $y+x^{2}$ não é $\mathcal{R}_{V}$-finito.

Uma $l$-transversal completa para $y+x^{2}$ é $y+x^{2}+a_{l} x^{l}$.

Seja $h=y+x^{2}+a_{l} x^{l} \operatorname{com} a_{l} \neq 0$. Então

$$
\begin{array}{ll}
\eta_{1} h=2 x^{2}+a_{l} l x^{l}+2 y & \eta_{2} h=2 z \\
\eta_{3} h=2 x^{3}+a_{l} l x^{l+1}+2 x y+3 a_{l} l x^{l-1} y & \eta_{4} h=2 x z+a_{l} l x^{l-1} z
\end{array}
$$

e $T \mathcal{R}_{V}(h)+\mathcal{M}^{l+2} \supseteq \mathcal{M}^{l+1}$ (para obter $x^{l+1}$ basta comparar $\eta_{1} h$ com $\eta_{3} h$ ) e portanto $y+x^{2}+a_{l} x^{l}$ é $(l+1)-\mathcal{R}_{V}$-determinado.

Como

$$
J^{l+1}\left(T \mathcal{R}_{V}^{1}(h)\right)+\mathbb{C}\left\{x^{l+1}\right\} \supseteq H^{l+1}
$$


temos que todos os $(l+1)$-jatos cujo $l$-jato é $y+x^{2}+a_{l} x^{l}$ são $\mathcal{R}_{V}$-equivalentes a $F=y+x^{2}+a_{l} x^{l}+a_{l+1} x^{l+1}$. Pode-se mostrar que $x^{l+1} \in T \mathcal{R}_{V}(F)$, e portanto pelo Lema de Mather podemos reduzir $F$ a $y+x^{2}+a_{l} x^{l}$. Logo $y+x^{2}+a_{l} x^{l}$ é $l-\mathcal{R}_{V^{-}}$ determinado para todo $a_{l} \neq 0$.

Caso (b): Classificação dos $l$-jatos cujo $(l-1)$-jato é $h(x, y, z)=y, l \geq 3$.

Temos $J^{l}\left(T \mathcal{R}_{V}^{1}(h)\right)+\mathbb{C}\left\{x^{l}\right\} \supseteq H^{l}$ e uma $l$-transversal completa para $y$ é $y+a_{l} x^{l}$.

Seja $h(x, y, z)=y+a_{l} x^{l}$. Temos que

$$
\begin{array}{ll}
\eta_{1} h=a_{l} l x^{l}+2 y & \eta_{2} h=2 z \\
\eta_{3} h=a_{l} l x^{l+1}+3 a_{l} l x^{l-1} y-4 x y & \eta_{4} h=a_{l} l x^{l-1} z
\end{array}
$$

e portanto $T \mathcal{R}_{V}(h)+\mathcal{M}^{l+2} \supseteq \mathcal{M}^{l+1}$, assim $y+a_{l} x^{l}$ é $(l+1)-\mathcal{R}_{V}$-determinado.

Examinemos então as deformações de $y+a_{l} x^{l}$ de ordem $l+1$.

Como $J^{l+1}\left(T \mathcal{R}_{V}^{1}(h)\right)+\mathbb{C}\left\{x^{l+1}\right\} \supseteq H^{l+1}$, uma $(l+1)$-transversal completa para $y+a_{l} x^{l}$ é $F=y+a_{l} x^{l}+a_{l+1} x^{l+1}$ Como $x^{l+1} \in T \mathcal{R}_{V}(F)$ para $\forall a_{l+1}$, segue-se pelo Lema de Mather que $F$ se reduz a $y+a_{l} x^{l}$. Portanto $y+a_{l} x^{l}$ é $l-\mathcal{R}_{V}$-determinado.

Caso (iii) Classificação dos germes cujo 1-jato é $z$.

Considere o germe $h(x, y, z)=z$. Então

$$
\begin{array}{ll}
\eta_{1} h=3 z & \eta_{2} h=x^{4}+4 x^{2} y+3 y^{2} \\
\eta_{3} h=0 & \eta_{4} h=2 x^{3} y+2 x y^{2}
\end{array}
$$

Aplicando o critério geométrico, vemos que $z$ não é $\mathcal{R}_{V}$-finito.

Como

$$
J^{2}\left(T \mathcal{R}_{V}^{1}(h)\right)+\mathbb{C}\left\{x^{2}, x y, y^{2}\right\} \supseteq H^{2}
$$

todos os 2-jatos cujo 1-jato é $z$ são $\mathcal{R}_{V}$-equivalentes a $z+a_{2} x^{2}+b x y+c y^{2}$.

Seja $h=z+a_{2} x^{2}+b x y+c y^{2}$. Então

$$
\begin{aligned}
& \eta_{1} h=2 a_{2} x^{2}+3 b x y+4 c y^{2}+3 z \\
& \eta_{2} h=2 b x z+x^{4}+4 x^{2} y+4 c y z+3 y^{2} \\
& \eta_{3} h=2 a_{2} x^{3}+6 a_{2} x y-3 b x^{2} y+3 b y^{2}-8 c x y^{2} \\
& \eta_{4} h=2 a_{2} x z+b y z+2 x^{3} y+2 x y^{2}
\end{aligned}
$$

Portanto $J^{2}\left(T \mathcal{R}_{V}\right)=<2 a_{2} x^{2}+3 b x y+4 c y^{2}+3 z, 2 b x z+4 c y z+3 y^{2}, 6 a_{2} x y+3 b y^{2}, 2 a_{2} x z+$ by $z$. Cálculos diretos mostram que $y^{2} \in j^{2}\left(T \mathcal{R}_{V}(h)\right), \forall a_{2}, b, c$ e se $a_{2} \neq 0, x y \in$ $j^{2}\left(T \mathcal{R}_{V}(h)\right), \forall b$ e c. Assim, pelo Lema de Mather temos: 
Para $a_{2} \neq 0, h=z+a_{2} x^{2}+b x y+c y^{2} \sim z+a_{2} x^{2}$.

Para $a_{2}=0, b \neq 0, h=z+b x y+c y^{2} \sim z+b x y$.

Para $a_{2}=b=0, h=z$.

Se $h=z+a_{2} x^{2}$ então $T \mathcal{R}_{V}(h)+\mathcal{M}^{4} \supseteq \mathcal{M}^{3}$, assim $z+a_{2} x^{2}$ é $3-\mathcal{R}_{V}$-determinado.

A condição $J^{3}\left(T \mathcal{R}_{V}^{1}(h)\right)+\mathbb{C}\left\{x^{3}\right\} \supseteq H^{3}$ se verifica e portanto o teorema da transversal completa garante que todos os 3 -jatos cujo 2 -jato é $z+a_{2} x^{2}$ são $\mathcal{R}_{V}$-equivalentes a $F=z+a_{2} x^{2}+a_{3} x^{3}$. Como $x^{3} \in T \mathcal{R}_{V}(F)$, segue do Lema de Mather que $F$ se reduz a $z+a_{2} x^{2}$. Logo $z+a_{2} x^{2}$ é $2-\mathcal{R}_{V}$-determinado.

Se $h=z+b x y$, temos que

$$
\begin{array}{ll}
\eta_{1} h=3 b x y+3 z & \eta_{2} h=2 b x z+x^{4}+4 x^{2} y+3 y^{2} \\
\eta_{3} h=-3 b x^{2} y+3 b y^{2} & \eta_{4} h=b y z+2 x^{3} y+2 x y^{2}
\end{array}
$$

Pelo critério geométrico, vemos que para $b \neq \pm 2$ e $b \neq 0 h=z+b x y$ é $\mathcal{R}_{V}$-finito. Uma forma pré-normal é dada por $z+b x y+r(x, y)$.

Se $j^{2} h=z$, então uma 3-transversal completa para $z$ é dada por $h=z+a_{3} x^{3}+b_{2} x^{2} y$. Logo,

$$
\begin{aligned}
& \eta_{1} h=3 a_{3} x^{3}+4 b_{2} x^{2} y+3 z \\
& \eta_{2} h=2 b_{2} x^{2} z+x^{4}+4 x^{2} y+3 y^{2} \\
& \eta_{3} h=3 a_{3} x^{4}+9 a_{3} x^{2} y-2 b_{2} x^{3} y+6 b_{2} x y^{2} \\
& \eta_{4} h=3 a_{3} x^{2} z+2 b_{2} x y z+2 x^{3} y+2 x y^{2}
\end{aligned}
$$

Aplicando o Lema de Mather, podemos reduzir $h$ a $z+a_{3} x^{3}$ para $a_{3} \neq 0$. Se $a_{3} \neq 0$ e $a_{3}^{2} \neq-4 / 27$ então $z+a_{3} x^{3}$ é finitamente determinado e uma forma pré-normal é dada por $z+a_{3} x^{3}+s(x)$. Para $a_{3}=0$ e $b_{2} \neq 0 h=z+b_{2} x^{2} y$ é finitamente determinado e uma forma pré-normal é $z+b_{2} x^{2} y+r(x, y)$.

Se $j^{3} h=z$, então uma 4-transversal completa para $z$ é dada por $h=z+a_{4} x^{4}+b_{3} x^{3} y$ e

$$
\begin{aligned}
& \eta_{1} h=4 a_{4} x^{4}+5 b_{3} x^{3} y+3 z \\
& \eta_{2} h=2 b_{3} x^{3} z+x^{4}+4 x^{2} y+3 y^{2} \\
& \eta_{3} h=4 a_{4} x^{5}+12 a_{4} x^{3} y-b_{3} x^{4} y+9 b_{3} x^{2} y^{2} \\
& \eta_{4} h=4 a_{4} x^{3} z+3 b_{3} x^{2} y z+2 x^{3} y+2 x y^{2}
\end{aligned}
$$

Cálculos diretos mostram que $x^{3} y \in J^{4}\left(T\left(\mathcal{R}_{V}(h)\right), \forall b_{3}\right.$, e pelo Lema de Mather, $h$ se reduz a $z+a_{4} x^{4}$ para $a_{4} \neq 0$. Se $a_{4} \neq 0$ então $z+a_{4} x^{4}$ é finitamente determinado e uma forma pré-normal é dada por $z+a_{4} x^{4}+s(x)$. 
Se $j^{4} h=z$, uma $l$-transversal completa para $z$ é $h=z+a_{l} x^{l}, l \geq 5$. Observemos que $x^{l-1} y$ não aparece na transvesal completa. (De fato, se $h=z$ basta olhar para $\eta_{2} h$ e $\left.\eta_{4} h\right)$.

$$
\begin{array}{ll}
\eta_{1} h=l a_{l} x^{l}+3 z & \eta_{2} h=x^{4}+4 x^{2} y+3 y^{2} \\
\eta_{3} h=l a_{l} x^{l+1}+3 l a_{l} x^{l-1} y & \eta_{4} h=l a_{l} x^{l-1} z+2 x^{3} y+2 x y^{2}
\end{array}
$$

Portanto,para $a_{l} \neq 0, h=z+a_{l} x^{l}$ é finitamente determinado e uma forma pré-normal é dada por $z+a_{l} x^{l}+s(x)$.

II- Seções de $S_{2}$.

Temos neste caso que $\Theta_{V}$ é gerado por

$$
\begin{aligned}
& \eta_{1}=(2 x, 6 y, 9 z) \\
& \eta_{2}=\left(0,2 z, x^{6}+4 x^{3} y+3 y^{2}\right) \\
& \eta_{3}=\left(x^{3}+3 y,-6 x^{2} y, 0\right) \\
& \eta_{4}=\left(z, 0,3\left(x^{5} y+x^{2} y^{2}\right)\right)
\end{aligned}
$$

Tomando-se o 1-jato $h=a x+b y+c z$, temos:

$$
\begin{aligned}
& \eta_{1} h=2 a x+6 b y+9 c z \\
& \eta_{2} h=2 b z+c\left(x^{6}+4 x^{3} y+3 y^{2}\right) \\
& \eta_{3} h=a\left(x^{3}+3 y\right)-6 b x^{2} y \\
& \eta_{4} h=a z+3 c\left(x^{5} y+x^{2} y^{2}\right)
\end{aligned}
$$

Portanto $J^{1}\left(T \mathcal{R}_{V}(h)\right)=<2 a x+6 b y+9 c z, 2 b z, 3 a y, a z>$. Assim, usando o Lema de Mather e mudanças de escalas temos de forma análoga ao caso anterior que:

Para $a \neq 0, h=a x+b y+c z \sim a x \sim x$.

Para $a=0, b \neq 0, h=b y+c z \sim b y \sim y$.

Para $a=b=0$ e $c \neq 0, h=c z \sim z$.

Portanto as órbitas em $J^{1}(3,1)$ da ação de $J^{1} \mathcal{R}_{V}$ são: $x, y, z$ e 0 .

Caso (i): Classificação dos germes $h$ cujo 1-jato é $x$.

Suponha que $h(x, y, z)=x$. Como no caso anterior, $x$ é $1-\mathcal{R}_{V}$-determinado.

Caso (ii): Classificação dos germes $h$ cujo 1-jato é $y$.

Suponha que $h(x, y, z)=y$. Então temos

$$
\begin{array}{ll}
\eta_{1} h=6 y & \eta_{2} h=2 z \\
\eta_{3} h=-6 x^{2} y & \eta_{4} h=0
\end{array}
$$


e $y$ não é $\mathcal{R}_{V}$-finito.

Como

$$
J^{2}\left(T \mathcal{R}_{V}^{1}(h)\right)+\mathbb{C}\left\{x^{2}\right\} \supseteq H^{2}
$$

uma 2-transversal completa para $y$ é dada pelo 2-jato $y+a_{2} x^{2}$.

Se $h=y+a_{2} x^{2}$, então temos

$$
\begin{array}{ll}
\eta_{1} h=4 a_{2} x^{2}+6 y & \eta_{2} h=2 z \\
\eta_{3} h=2 a_{2} x^{4}+6 a_{2} x y-6 x^{2} y & \eta_{4} h=2 a_{2} x z
\end{array}
$$

e $T \mathcal{R}_{V}(h) \supseteq \mathcal{M}^{3}$. Assim se $a_{2} \neq 0, y+a_{2} x^{2}$ é $3-\mathcal{R}_{V}$-determinado.

Uma 3-transversal completa para $y+a_{2} x^{2}$ é $F=y+a_{2} x^{2}+a_{3} x^{3}$. Como $x^{3} \in$ $T \mathcal{R}_{V}(F)$, pelo Lema de Mather, $F$ se reduz a $y+a_{2} x^{2}$. Portanto $y+a_{2} x^{2}$ é $2-\mathcal{R}_{V^{*}}$ determinado se $a_{2} \neq 0$.

Para $a_{2}=0$ e $h=y$ temos que:

$$
J^{3}\left(T \mathcal{R}_{V}^{1}(h)\right)+\mathbb{C}\left\{x^{3}\right\} \supseteq H^{3}
$$

e uma 3-transversal completa para $y$ é $y+a_{3} x^{3}$.

Para $h=y+a_{3} x^{3}$, os geradores $\eta_{i} h$ são:

$$
\begin{array}{ll}
\eta_{1} h=6 a_{3} x^{3}+6 y & \eta_{2} h=2 z \\
\eta_{3} h=3 a_{3} x^{5}+\left(9 a_{3}-6\right) x^{2} y & \eta_{4} h=3 a_{3} x^{2} z
\end{array}
$$

Vemos portanto que $y+a_{3} x^{3}$ é $\mathcal{R}_{V}$-determinado se, e somente se, $a_{3} \neq 0$ e $a_{3} \neq 1$. Neste caso $T \mathcal{R}_{V}(h) \supset \mathcal{M}^{5}$ e, portanto, $h$ é 5- $\mathcal{R}_{V}$-determinado. Como

$$
J^{4}\left(T \mathcal{R}_{V}^{1}(h)\right)+\mathbb{C}\left\{x^{4}\right\} \supseteq H^{4}
$$

uma 4-transversal completa para $y+a_{3} x^{3}$ é $F=y+a_{3} x^{3}+a_{4} x^{4}$. Temos que $x^{4} \notin$ $T \mathcal{R}_{V}(F)$. Seja $h=y+a_{3} x^{3}+a_{4} x^{4}$. A seguinte relação se verifica

$$
J^{5}\left(T \mathcal{R}_{V}^{1}(h)\right)+\mathbb{C}\left\{x^{5}\right\} \supseteq H^{5}
$$

e portanto, uma 5-transversal completa para $y+a_{3} x^{3}+a_{4} x^{4}$ é $F=y+a_{3} x^{3}+a_{4} x^{4}+a_{5} x^{5}$. Como $x^{5} \in T \mathcal{R}_{V}(F)$, segue-se pelo Lema de Mather que $F$ se reduz a $y+a_{3} x^{3}+a_{4} x^{4}$, que é $4-\mathcal{R}_{V}$-determinado. 
Para $a_{3}=1$, seja $h=y+x^{3}$. Então

$$
\begin{array}{ll}
\eta_{1} h=6 x^{3}+6 y & \eta_{2} h=2 z \\
\eta_{3} h=3 x^{5}+3 x^{2} y & \eta_{4} h=3 x^{2} z
\end{array}
$$

e portanto $y+x^{3}$ não é $\mathcal{R}_{V \text {-finito. }}$

Uma l-transversal completa para $y+x^{3}$ é $y+x^{3}+a_{l} x^{l}$ pois

$$
J^{l}\left(T \mathcal{R}_{V}^{1}(h)\right)+\mathbb{C}\left\{x^{l}\right\} \supseteq H^{l}
$$

Seja $h=y+x^{3}+a_{l} x^{l}$. Então

$$
\begin{array}{ll}
\eta_{1} h=6 x^{3}+2 l a_{l} x^{l}+6 y & \eta_{2} h=2 z \\
\eta_{3} h=3 x^{5}+3 x^{2} y+l a_{l} x^{l+2}+3 l a_{l} x^{l-1} y & \eta_{4} h=3 x^{2} z+l a_{l} x^{l-1} z
\end{array}
$$

e cálculos mostram que $T \mathcal{R}_{V}(h)+\mathcal{M}^{l+3} \supseteq \mathcal{M}^{l+2}$ (para obter $x^{l+2}$ basta observar que $\left.2 \eta_{3} h-x^{2} \eta_{1} h=6 l a_{l} x^{l-1} y\right)$. Logo $y+x^{3}+a_{l} x^{l}$ é $(l+2)-\mathcal{R}_{V}$-determinado.

Analogamente ao caso anterior, obtemos que uma $(l+1)$-transversal completa para $y+x^{3}+a_{l} x^{l}$ é $F=y+x^{3}+a_{l} x^{l}+a_{l+1} x^{l+1}$ que é $(l+1)-\mathcal{R}_{V^{-}}$determinado.

Se $a_{3}=0$ e $j^{l-1} h=y$, temos que

$$
J^{l}\left(T \mathcal{R}_{V}^{1}(h)\right)+\mathbb{C}\left\{x^{l}\right\} \supseteq H^{l}
$$

e uma $l$-transversal completa para $y$ é $y+a_{l} x^{l}, l \geq 4$.

Seja $h=y+a_{l} x^{l}$. Temos que

$$
\begin{array}{ll}
\eta_{1} h=2 l a_{l} x^{l}+6 y & \eta_{2} h=2 z \\
\eta_{3} h=l a_{l} x^{l+2}+3 l a_{l} x^{l-1} y-6 x^{2} y & \eta_{4} h=l a_{l} x^{l-1} z
\end{array}
$$

Portanto, $T \mathcal{R}_{V}(h)+\mathcal{M}^{l+3} \supseteq \mathcal{M}^{l+2}$ e $y+a_{l} x^{l}$ é $(l+2)-\mathcal{R}_{V}$-determinado.

Como

$$
J^{l+1}\left(T \mathcal{R}_{V}^{1}(h)\right)+\mathbb{C}\left\{x^{l+1}\right\} \supseteq H^{l+1}
$$

uma $(l+1)$-transversal completa para $y+a_{l} x^{l}$ é $F=y+a_{l} x^{l}+a_{l+1} x^{l+1}$, que é $(l+1)-\mathcal{R}_{V^{-}}$ determinado.

Caso (iii): Classificação dos germes $h$ cujo 1-jato é $z$. 
Suponha que $h(x, y, z)=z$. Então temos

$$
\begin{array}{ll}
\eta_{1} h=9 z & \eta_{2} h=x^{6}+4 x^{3} y+3 y^{2} \\
\eta_{3} h=0 & \eta_{4} h=3 x^{5} y+3 x^{2} y^{2}
\end{array}
$$

e, portanto $z$ não é $\mathcal{R}_{V}$-finito.

Como

$$
J^{2}\left(T \mathcal{R}_{V}^{1}(h)\right)+\mathbb{C}\left\{x^{2}, x y, y^{2}\right\} \supseteq H^{2}
$$

uma 2-transversal completa para $z$ é $z+a_{2} x^{2}+b x y+c y^{2}$.

Seja $h=z+a_{2} x^{2}+b x y+c y^{2}$. Então temos

$$
\begin{aligned}
& \eta_{1} h=4 a_{2} x^{2}+8 b x y+12 c y^{2}+9 z \\
& \eta_{2} h=2 b x z+x^{6}+4 x^{3} y+4 c y z+3 y^{2} \\
& \eta_{3} h=2 a_{2} x^{4}+6 a_{2} x y-5 b x^{3} y+3 b y^{2}-12 c x^{2} y^{2} \\
& \eta_{4} h=2 a_{2} x z+b y z+2 x^{5} y+3 x^{2} y^{2}
\end{aligned}
$$

Portanto $J^{2}\left(T \mathcal{R}_{V}\right)=<4 a_{2} x^{2}+8 b x y+12 c y^{2}+9 z, 2 b x z+4 c y z+3 y^{2}, 6 a_{2} x y+3 b y^{2}, 2 a_{2} x z+$ by $z>$ Temos $j^{2} x \eta_{1} h=9 x z, j^{2} y \eta_{1} h=9 y z, j^{2} \eta_{2} h=2 b x z+4 c y z+3 y^{2}$ e $j^{2} \eta_{3} h=$ $6 a_{2} x y+3 b y^{2}$. Então $a_{2} x y, y^{2} \in j^{2}\left(T \mathcal{R}_{V}(h)\right)$ e pelo Lema de Mather temos:

Para $a_{2} \neq 0, h=z+a_{2} x^{2}+b x y+c y^{2} \sim z+a_{2} x^{2}$.

Para $a_{2}=0, b \neq 0, h=z+b x y+c y^{2} \sim z+b x y$.

Para $a_{2}=b=0, h=z$.

Se $h=z+a_{2} x^{2}$, com cálculos análogos ao caso anterior podemos mostrar que $h$ é $2-\mathcal{R}_{V}$-determinado.

Se $a_{2}=0$ e $b \neq 0$ tomemos $h=z+b x y$. Temos que

$$
\begin{array}{ll}
\eta_{1} h=8 b x y+9 z & \eta_{2} h=2 b x z+x^{6}+4 x^{3} y+3 y^{2} \\
\eta_{3} h=-5 b x^{3} y+3 b y^{2} & \eta_{4} h=b y z+3 x^{5} y+3 x^{2} y^{2}
\end{array}
$$

Para $b \neq 0, z+b x y$ é finitamente determinado e uma forma pré-normal é dada por $z+b x y+r(x, y)$

Se $j^{2} h=z$, uma 3-transversal completa para $z$ é $h=z+a_{3} x^{3}+b_{2} x^{2} y$, então

$$
\begin{aligned}
& \eta_{1} h=6 a_{3} x^{3}+10 b_{2} x^{2} y+9 z \\
& \eta_{2} h=2 b_{2} x^{2} z+x^{6}+4 x^{3} y+3 y^{2} \\
& \eta_{3} h=3 a_{3} x^{5}+9 a_{3} x^{2} y-4 b_{2} x^{4} y+6 b x y^{2} \\
& \eta_{4} h=3 a_{3} x^{2} z+b_{2} x y z+3 x^{5} y+3 x^{2} y^{2}
\end{aligned}
$$


Aplicando o Lema de Mather, podemos reduzir $h$ a $z+a_{3} x^{3}$ para $a_{3} \neq 0$. Se $a_{3} \neq 0$, $z+a_{3} x^{3}$ é finitamente determinado e uma forma pré-normal é dada por $z+a_{3} x^{3}+s(x)$. Para $a_{3}=0$ e $b_{2} \neq 0 h=z+b_{2} x^{2} y$ é finitamente determinado e uma forma pré-normal é $z+b_{2} x^{2} y+r(x, y)$.

Se $j^{3} h=z$, uma 4-transversal completa para $z$ é dada por $h=z+a_{4} x^{4}+b_{3} x^{3} y$, então, como acima, $h$ se reduz a $z+a_{4} x^{4}$ para $a_{4} \neq 0$. Se $a_{4} \neq 0$ então $z+a_{4} x^{4}$ é finitamente determinado e uma forma pré-normal é dada por $z+a_{4} x^{4}+s(x)$. Para $a_{4}=0$ e $b_{3} \neq 0, h=z+b_{3} x^{3} y$ é finitamente determinado e uma forma pré-normal é $z+b_{3} x^{3} y+r_{3}(x, y)$.

Se $j^{4} h=z$, uma 5-transversal completa para $z$ é dada por $h=z+a_{5} x^{5}+b_{4} x^{4} y$. Se $a_{5} \neq 0, h$ se reduz a $z+a_{5} x^{5}$, que é finitamente determinado. Uma forma pré-normal é dada por $z+a_{5} x^{5}+s(x)$. Para $a_{5}=0$ e $b_{4} \neq 0, h=z+b_{4} x^{4} y$ é finitamente determinado e uma forma pré-normal é $z+b_{4} x^{4} y+r(x, y)$.

Se $j^{5} h=z$, uma 6 -transversal completa para $z$ é dada por $h=z+a_{6} x^{6}+b_{5} x^{5} y$. Então

$$
\begin{aligned}
& \eta_{1} h=12 a_{6} x^{6}+16 b_{5} x^{5} y+9 z \\
& \eta_{2} h=2 b_{5} x^{5} z+x^{6}+4 x^{3} y+3 y^{2} \\
& \eta_{3} h=6 a_{6} x^{8}+18 a_{6} x^{5} y-b_{5} x^{7} y+15 b_{5} x^{4} y^{2} \\
& \eta_{4} h=6 a_{6} x^{5} z+5 b_{5} x^{4} y z+3 x^{5} y+3 x^{2} y^{2}
\end{aligned}
$$

Cálculos diretos mostram que $x^{5} y \in J^{6}\left(T\left(\mathcal{R}_{V}(h)\right), \forall b_{5}\right.$, e pelo Lema de Mather, $h$ se reduz a $z+a_{6} x^{6}$ para $a_{6} \neq 0$. Se $a_{6} \neq 0$ então $z+a_{6} x^{6}$ é finitamente determinado $\mathrm{e}$ uma forma pré-normal é dada por $z+a_{6} x^{6}+s(x)$.

Se $j^{6} h=z$, uma $l$-transversal completa para $z$ é $h=z+a_{l} x^{l}, l \geq 7$, observamos que $x^{l-1} y$ não aparece na transvesal completa. (De fato, se $h=z$ basta olhar para $\eta_{2} h$ e $\left.\eta_{4} h\right)$. Então

$$
\begin{array}{ll}
\eta_{1} h=2 l a_{l} x^{l}+9 z & \eta_{2} h=x^{6}+4 x^{3} y+3 y^{2} \\
\eta_{3} h=l a_{l} x^{l+2}+3 l a_{l} x^{l-1} y & \eta_{4} h=l a_{l} x^{l-1} z+3 x^{5} y+3 x^{2} y^{2}
\end{array}
$$

Portanto,para $a_{l} \neq 0, h=z+a_{l} x^{l}$ é finitamente determinado e uma forma pré-normal é dada por $z+a_{l} x^{l}+s(x)$.

(III) Seção de $S_{3}$. 
Temos neste caso que $\Theta_{V}$ é gerado por

$$
\begin{aligned}
& \eta_{1}=(2 x, 8 y, 12 z) \\
& \eta_{2}=\left(0,2 z, x^{8}+4 x^{4} y+3 y^{2}\right) \\
& \eta_{3}=\left(x^{4}+3 y,-8 x^{3} y, 0\right) \\
& \eta_{4}=\left(z, 0,4\left(x^{7} y+x^{3} y^{2}\right)\right)
\end{aligned}
$$

Como nos casos anteriores as órbitas em $J^{1}(3,1)$ da ação de $J^{1} \mathcal{R}_{V}$ são: $x, y, z$ e 0 .

Caso (i): Classificação dos germes $h$ cujo 1-jato é $x$.

Assim como nos casos anteriores, $T \mathcal{R}_{V}(h) \supset \mathcal{M}^{1}$ e $x$ é $1-\mathcal{R}_{V}$-determinado.

Caso (ii): Classificação dos germes $h$ cujo 1-jato é $y$.

Suponhamos que $j^{l-1} h=y, l \geq 2$. Então, uma $l$-transversal completa para $y$ é $y+a_{l} x^{l}$.

Se $h=y+a_{l} x^{l}$. Temos que

$$
\begin{array}{ll}
\eta_{1} h=2 l a_{l} x^{l}+8 y & \eta_{2} h=2 z \\
\eta_{3} h=l a_{l} x^{l+3}+3 l a_{l} x^{l-1} y-8 x^{3} y & \eta_{4} h=l a_{l} x^{l-1} z
\end{array}
$$

As equações $\eta_{1} h$ e $\eta_{3} h$ são linearmente independentes sempre que $l \neq 4$ e $a_{l} \neq 0$ ou $l=4, a_{4} \neq 0$ e $a_{4} \neq 1$. De fato, quando $l=4$ e $a_{4}=1, x^{3} \eta_{1} h=2 \eta_{3} h$.

Nos demais casos, isto é, $l \neq 4$ e $a_{l} \neq 0$ ou $l=4, a_{4} \neq 0$ e $a_{4} \neq 1$ podemos mostrar que

$$
T \mathcal{R}_{V}(h)+\mathcal{M}^{l+4} \supseteq \mathcal{M}^{l+3}
$$

portanto $y+a_{l} x^{l}$ é $(l+3)-\mathcal{R}_{V}$-determinado.

Para $l=2$ e 3 é fácil melhorar esta estimativa. Com efeito, para $l=2, h=y+a_{2} x^{2}$ é 2-determinado, $\forall a_{2} \neq 0$. Para $l=3$, uma transversal completa para o 4 -jato de $y+a_{3} x^{3}$ é $h=y+a_{3} x^{3}+a_{4} x^{4}$, que é 4-determinado, $\forall a_{3} \neq 0$.

Para $l=4, a_{4} \neq 0, a_{4} \neq 1$, aplicando o método das transversais completas ao 5-jato de $y+a_{4} x^{4}$ obtemos $y+a_{4} x^{4}+a_{5} x^{5}$, e ao 6-jato de $y+a_{4} x^{4}+a_{5} x^{5}$, obtemos $h=y+a_{4} x^{4}+a_{5} x^{5}+a_{6} x^{6}$, que é 6-determinado $\forall a_{4} \neq 0, a_{4} \neq 1$.

Para $l \geq 5, a_{l} \neq 0$, usando a estimativa acima para a ordem de determinação finita e aplicando sucessivamente o método das transversais completas, obtemos que $h$ se reduz a $y+a_{l} x^{l}+a_{l+1} x^{l+1}+a_{l+2} x^{l+2}$, que é $l+2$-determinado, $\forall a_{l} \neq 0$.

Falta analisar o caso $l=4, a_{4}=1, h=y+x^{4}$. 
Se $h=y+x^{4}$. Temos que

$$
\begin{array}{ll}
\eta_{1} h=8 x^{4}+8 y & \eta_{2} h=2 z \\
\eta_{3} h=4 x^{7}+4 x^{3} y & \eta_{4} h=4 x^{3} z
\end{array}
$$

e portanto $y+x^{4}$ não é $\mathcal{R}_{V}$-determinado.

Suponhamos que $j^{l-1} h=y+x^{4}$. Como

$$
J^{l}\left(T \mathcal{R}_{V}^{1}(h)\right)+\mathbb{C}\left\{x^{l}\right\} \supseteq H^{l}
$$

uma $l$-transversal completa para $y+x^{4}$ é $y+x^{4}+a_{l} x^{l}, l \geq 5$.

A seguinte relação se verifica para o espaço tangente de $y+x^{4}+a_{l} x^{l}$

$$
T \mathcal{R}_{V}(h)+\mathcal{M}^{l+4} \supseteq \mathcal{M}^{l+3}
$$

e portanto, $y+x^{4}+a_{l} x^{l}$ é $(l+3)-\mathcal{R}_{V}$-determinado.

Aqui, novamente, basta aplicar sucessivamente o método das transversais completas para mostrar que se $j^{l-1} h=y+x^{4}, h$ é equivalente a $y+x^{4}+a_{l} x^{l}+a_{l+1} x^{l+1}+a_{l+2} x^{l+2}$, que é $(l+2)$ - $\mathcal{R}_{V}$-determinado.

Caso (iii): Classificação dos germes $h$ cujo 1-jato é $z$.

Suponha que $h(x, y, z)=z$. Então temos

$$
\begin{array}{ll}
\eta_{1} h=12 z & \eta_{2} h=x^{8}+4 x^{4} y+3 y^{2} \\
\eta_{3} h=0 & \eta_{4} h=4 x^{7} y+4 x^{3} y^{2}
\end{array}
$$

e portanto $h=z$ não é $\mathcal{R}_{V}$-finito.

Como

$$
J^{2}\left(T \mathcal{R}_{V}^{1}(h)\right)+\mathbb{C}\left\{x^{2}, x y, y^{2}\right\} \supseteq H^{2}
$$

uma 2-transversal completa para $z$ é $z+a_{2} x^{2}+b x y+c y^{2}$.

Se $h=z+a_{2} x^{2}+b x y+c y^{2}$. Então temos

$$
\begin{aligned}
& \eta_{1} h=4 a_{2} x^{2}+10 b x y+16 c y^{2}+12 z \\
& \eta_{2} h=2 b x z+x^{8}+4 x^{4} y+4 c y z+3 y^{2} \\
& \eta_{3} h=2 a_{2} x^{5}+6 a_{2} x y-7 b x^{4} y+3 b y^{2}-16 c x^{3} y^{2} \\
& \eta_{4} h=2 a_{2} x z+b y z+4 x^{7} y+4 x^{3} y^{2}
\end{aligned}
$$

Portanto $J^{2}\left(T \mathcal{R}_{V}\right)=<4 a_{2} x^{2}+10 b x y+16 c y^{2}+12 z, 2 b x z+4 c y z+3 y^{2}, 6 a_{2} x y+$ $3 b y^{2}, 2 a_{2} x z+b y z>$. Temos $j^{2} x \eta_{1} h=12 x z, j^{2} y \eta_{1} h=12 y z, j^{2} \eta_{2} h=2 b x z+4 c y z+3 y^{2}$ e $j^{2} \eta_{3} h=6 a_{2} x y+3 b y^{2}$. Então $a_{2} x y, y^{2} \in j^{2}\left(T \mathcal{R}_{V}(h)\right)$ e pelo Lema de Mather temos: 
Para $a_{2} \neq 0, h=z+a_{2} x^{2}+b x y+c y^{2} \sim z+a_{2} x^{2}$.

Para $a_{2}=0, b \neq 0, h=z+b x y+c y^{2} \sim z+b x y$.

Para $a_{2}=b=0, h=z$.

Se $h=z+a_{2} x^{2}$ podemos mostrar que $h$ é $2-\mathcal{R}_{V^{\prime \prime}}$ determinado.

Se $a_{2}=0$ e $b \neq 0, h=z+b x y$ é finitamente determinado, como podemos verificar pelo critério geométrico, e uma forma pré normal é dada por $z+b x y+r(x, y)$.

Se $b=0$, seja $h=z$.

Como

$$
J^{l}\left(T \mathcal{R}_{V}^{1}(h)\right)+\mathbb{C}\left\{x^{l}, x^{l-1} y\right\} \supseteq H^{l}
$$

uma $l$-transversal completa para $z$ é $z+a_{l} x^{l}+b_{l-1} x^{l-1} y$ se $3 \leq l \leq 8$ e $z+a_{l} x^{l}$ se $l \geq 9$.

Para $h=z+a_{l} x^{l}+b_{l-1} x^{l-1} y$, os geradores do espaço tangente são dados por:

$$
\begin{aligned}
& \eta_{1} h=2 l a_{l} x^{l}+(2 l+6) b_{l-1} x^{l-1} y+12 z \\
& \eta_{2} h=2 b_{l-1} x^{l-1} z+x^{8}+4 x^{4} y+3 y^{2} \\
& \eta_{3} h=l a_{l} x^{l+3}+3 l a_{l} x^{l-1} y+(l-9) b_{l-1} x^{l+2} y+(3 l-3) b_{l-1} x^{l-2} y^{2} \\
& \eta_{4} h=l a_{l} x^{l-1} z+(l-1) b_{l-1} x^{l-2} y z+4 x^{7} y+4 x^{3} y^{2}
\end{aligned}
$$

Para $a_{l} \neq 0, x^{l-1} y \in T \mathcal{R}_{V}(h)$, e do Lema de Mather segue que $h$ se reduz a $z+a_{l} x^{l}$.

Determinando as soluções $\eta_{1} h=\eta_{2} h=\eta_{3} h=\eta_{4} h=0$ e aplicando o critério geométrico vemos que para $a_{l} \neq 0, l \neq 6, z+a_{l} x^{l}$ é finitamente determinado e para $a_{6} \neq 0$ e $a_{6}^{2} \neq-4 / 27, z+a_{6} x^{6}$ é finitamente determinado. Uma forma pré-normal para $h=z+a_{l} x^{l}$ é dada por $z+a_{l} x^{l}+s(x)$.

Para $a_{l}=0$, seja $h=z+b_{l-1} x^{l-1} y \operatorname{com} l \leq 7$ então, pelo critério geométrico, para $b_{l-1} \neq 0$ e $b_{l-1} \neq \pm 2, z+b_{l-1} x^{l-1} y$ é finitamente determinado e uma forma pré-normal é dada por $z+b_{l-1} x^{l-1} y+r(x, y)$.

(IV) O Caso Geral: Seções de $S_{k}$

Temos neste caso que $\Theta_{V}$ é gerado por

$$
\begin{aligned}
& \eta_{1}=(2 x, 2(k+1) y, 3(k+1) z) \\
& \eta_{2}=\left(0,2 z, x^{2(k+1)}+4 x^{k+1} y+3 y^{2}\right) \\
& \eta_{3}=\left(x^{k+1}+3 y,-2(k+1) x^{k} y, 0\right) \\
& \eta_{4}=\left(z, 0,(k+1)\left(x^{2 k+1} y+x^{k} y^{2}\right)\right)
\end{aligned}
$$

De forma análoga aos casos anteriores, obtém-se que as órbitas em $J^{1}(3,1)$ da ação de $J^{1} \mathcal{R}_{V}$ são: $x, y, z$ e 0. 
Caso (i): Classificação dos germes $h$ cujo 1-jato é $x$.

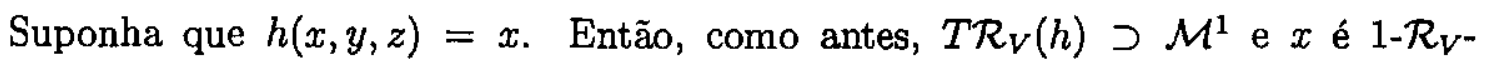
determinado.

Caso (ii): Classificação dos germes $h$ cujo 1-jato é $y$.

Os geradores do espaço tangente são:

$$
\eta_{1} h=2(k+1) y, \quad \eta_{2} h=2 z, \quad \eta_{3} h=-2(k+1) x^{3} y \text { e } \quad \eta_{4} h=0,
$$

e uma $l$-transversal completa para $j^{l-1} h=y$ é $y+a_{l} x^{l}$.

Seja $h=y+a_{l} x^{l}$. Então temos

$$
\begin{array}{ll}
\eta_{1} h=2 l a_{l} x^{l}+2(k+1) y & \eta_{2} h=2 z \\
\eta_{3} h=l a_{l} x^{k+l}+3 l a_{l} x^{l-1} y-2(k+1) x^{k} y & \eta_{4} h=l a_{l} x^{l-1} z
\end{array}
$$

Determinando as soluções de $\eta_{1} h=\eta_{2} h=\eta_{3} h=\eta_{4} h=0$ e aplicando o critério geométrico, temos:

(a) Se $l=k+1, a_{k+1} \neq 0$ e $a_{k+1} \neq 1$ então $h=y+a_{k+1} x^{k+1}$ é finitamente- $\mathcal{R}_{V^{-}}$ determinado.

(b) Se $l \neq(k+1)$ e $a_{l} \neq 0$ então $h=y+a_{l} x^{l}$ é finitamente- $\mathcal{R}_{V}$-determinado.

Caso (a): Classificação dos germes cujo $(k+1)$-jato é $h=y+a_{k+1} x^{k+1}$. Neste caso temos:

$$
\begin{aligned}
& \eta_{1} h=2(k+1) a_{k+1} x^{k+1}+2(k+1) y \\
& \eta_{2} h=2 z \\
& \eta_{3} h=(k+1) a_{k+1} x^{2 k+1}+\left(3(k+1) a_{k+1}-2(k+1)\right) x^{k} y \\
& \eta_{4} h=(k+1) a_{k+1} x^{k} z
\end{aligned}
$$

Então $T \mathcal{R}_{V}(h) \supseteq \mathcal{M}^{2 k+1}$, se $a_{k+1} \neq 0$ e $a_{k+1} \neq 1$, assim $y+a_{k+1} x^{k+1}$ é $(2 k+1)-\mathcal{R}_{V^{-}}$ determinado. Usando sucessivamente o método das transversais completa obteremos $F=y+a_{k+1} x^{k+1}+a_{k+2} x^{k+2}+\ldots+a_{2 k} x^{2 k}+a_{2 k+1} x^{2 k+1}$, como $x^{2 k+1} \in T \mathcal{R}_{V}(F)$, segue-se pelo Lema de Mather que $F$ se reduz a $y+a_{k+1} x^{k+1}+a_{k+2} x^{k+2}+\ldots+a_{2 k} x^{2 k}$.

Para $a_{k+1}=1$, seja $h=y+x^{k+1}$. Então temos

$$
\begin{array}{ll}
\eta_{1} h=2(k+1) x^{k+1}+2(k+1) y & \eta_{2} h=2 z \\
\eta_{3} h=(k+1) x^{2 k+1}+(k+1) x^{k} y & \eta_{4} h=(k+1) x^{k} z
\end{array}
$$

portanto, pelo critério geométrico, $y+x^{k+1}$ não é $\mathcal{R}_{V}$-finito. 
Como

$$
J^{l}\left(T \mathcal{R}_{V}^{1}(h)\right)+\mathbb{C}\left\{x^{l}\right\} \supseteq H^{l}
$$

uma $l$-transversal completa para $y+x^{k+1}$ é $y+x^{k+1}+a_{l} x^{l}, l>(k+1)$.

Seja $h=y+x^{k+1}+a_{l} x^{l} \operatorname{com} a_{l} \neq 0$. Então

$$
\begin{aligned}
& \eta_{1} h=2(k+1) x^{k+1}+2(k+1) y+2 a_{l} l x^{l} \\
& \eta_{2} h=2 z \\
& \eta_{3} h=(k+1) x^{2 k+1}+(k+1) x^{k} y+l a_{l} x^{k+l}+3 l a_{l} x^{l-1} y \\
& \eta_{4} h=(k+1) x^{k} z+l a_{l} x^{l-1} z
\end{aligned}
$$

Portanto $T \mathcal{R}_{V}(h)+\mathcal{M}^{k+l+1} \supseteq \mathcal{M}^{k+l}$, assim $y+x^{k+1}+a_{l} x^{l}$ é $(k+l)-\mathcal{R}_{V}$-determinado. Usando sucessivamente o método das transversais completa obteremos $F=y+x^{k+1}+$ $a_{l} x^{l}+\ldots+a_{k+l-1} x^{k+l-1}+a_{k+l} x^{k+l}$, como $x^{k+l} \in T \mathcal{R}_{\mathcal{V}}(F)$, segue-se pelo Lema de Mather que $F$ se reduz a $y+x^{k+1}+a_{l} x^{l}+\ldots+a_{k+l-1} x^{k+l-1}$.

Caso (b): Classificação dos germes cujo $l$-jato é $h=y+a_{l} x^{l}$ com $l \neq(k+1)$. Neste caso temos,

$$
\begin{array}{ll}
\eta_{1} h=2 l a_{l} x^{l}+2(k+1) y & \eta_{2} h=2 z \\
\eta_{3} h=l a_{l} x^{k+l}+3 l a_{l} x^{l-1} y-2(k+1) x^{k} y & \eta_{4} h=l a_{l} x^{l-1} z
\end{array}
$$

Assim, se $l>(k+1)$ então $T \mathcal{R}_{V}(h)+\mathcal{M}^{k+l+1} \supseteq \mathcal{M}^{k+l}$ e $y+a_{l} x^{l}$ é $(k+l)-\mathcal{R}_{V^{-}}$ determinado. Usando sucessivamente o método das transversais completa obteremos $F=y+a_{l} x^{l}+\ldots+a_{k+l-1} x^{k+l-1}+a_{k+l} x^{k+l}$, como $x^{k+l} \in T \mathcal{R}_{V}(F)$, segue-se pelo Lema de Mather que $F$ se reduz a $y+x^{k+1}+a_{l} x^{l}+\ldots+a_{k+l-1} x^{k+l-1}$.

Se $l<(k+1)$ então $T \mathcal{R}_{V}(h)+\mathcal{M}^{2 l} \supseteq \mathcal{M}^{2 l-1}$ e $y+a_{l} x^{l}$ é $(2 l-1)$ - $\mathcal{R}_{V \text {-determinado. }}$ Usando sucessivamente o método das transversais completa obteremos $F=y+a_{l} x^{l}+$ $\ldots+a_{2 l-2} x^{2 l-2}+a_{2 l-1} x^{2 l-1}$, como $x^{2 l-1} \in T \mathcal{R}_{V}(F)$, segue-se pelo Lema de Mather que $F$ se reduz a $y+a_{l} x^{l}+\ldots+a_{2 l-2} x^{2 l-2}$.

Caso (iii): Classificação dos germes $h$ cujo 1-jato é $z$.

Suponha que $h(x, y, z)=z$. Então temos

$$
\begin{array}{ll}
\eta_{1} h=3(k+1) z & \eta_{2} h=x^{2 k+2}+4 x^{k+1} y+3 y^{2} \\
\eta_{3} h=0 & \eta_{4} h=(k+1) x^{2 k+1} y+(k+1) x^{k} y^{2}
\end{array}
$$

Portanto, pelo critério geométrico, $h=z$ não é $\mathcal{R}_{V}$-finito. 
Como

$$
J^{2}\left(T \mathcal{R}_{V}^{1}(h)\right)+\mathbb{C}\left\{x^{2}, x y, y^{2}\right\} \supseteq H^{2}
$$

uma 2-transversal completa para $z$ é $h=z+a_{2} x^{2}+b x y+c y^{2}$. Os geradores do espaço tangente são:

$$
\begin{aligned}
& \eta_{1} h=4 a_{2} x^{2}+(4+2 k) b x y+4(k+1) c y^{2}+3(k+1) z \\
& \eta_{2} h=2 b x z+x^{2 k+2}+4 x^{k+1} y+4 c y z+3 y^{2} \\
& \eta_{3} h=2 a_{2} x^{k+2}+6 a_{2} x y-(2 k+1) b x^{k+1} y+3 b y^{2}-4(k+1) c x^{k} y^{2} \\
& \eta_{4} h=2 a_{2} x z+b y z+(k+1) x^{2 k+1} y+(k+1) x^{k} y^{2}
\end{aligned}
$$

Assim $J^{2}\left(T \mathcal{R}_{V}\right)=<4 a_{2} x^{2}+(4+2 k) b x y+4(k+1) c y^{2}+3(k+1) z, 2 b x z+4 c y z+$ $3 y^{2}, 6 a_{2} x y+3 b y^{2}, 2 a_{2} x z+b y z>$ Temos $j^{2} x \eta_{1} h=3(k+1) x z, j^{2} y \eta_{1} h=3(k+1) y z$, $j^{2} \eta_{2} h=2 b x z+4 c y z+3 y^{2}$ e $j^{2} \eta_{3} h=6 a_{2} x y+3 b y^{2}$. Então $a_{2} x y, y^{2} \in j^{2}\left(T \mathcal{R}_{V}(h)\right) \mathrm{e}$ pelo Lema de Mather temos:

Para $a_{2} \neq 0, h=z+a_{2} x^{2}+b x y+c y^{2} \sim z+a_{2} x^{2}$.

Para $a_{2}=0, b \neq 0, h=z+b x y+c y^{2} \sim z+b x y$.

Para $a_{2}=b=0, h=z$.

Se $a_{2} \neq 0, h=z+a_{2} x^{2}$ é $2-\mathcal{R}_{V}$-determinado.

Se $a_{2}=0$ e $b \neq 0$ tomemos $h=z+b x y$. Temos que

$$
\begin{aligned}
& \eta_{1} h=(4+2 k) b x y+3(k+1) z \\
& \eta_{2} h=2 b x z+x^{2 k+2}+4 x^{k+1} y+3 y^{2} \\
& \eta_{3} h=-(2 k+1) b x^{k+1} y+3 b y^{2} \\
& \eta_{4} h=b y z+(k+1) x^{2 k+1} y+(k+1) x^{k} y^{2}
\end{aligned}
$$

Portanto para $b \neq 0, h$ é finitamente determinado e uma forma pré normal é dada por $z+b x y+r(x, y)$. Se $b=0$, seja $j^{l-1} h=z, l \geq 3$.

Como

$$
J^{l}\left(T \mathcal{R}_{V}^{1}(h)\right)+\mathbb{C}\left\{x^{l}, x^{l-1} y\right\} \supseteq H^{l}
$$

uma $l$-transversal completa para $z$ é $z+a_{l} x^{l}+b_{l-1} x^{l-1} y$ se $3 \leq l \leq(2 k+2)$ e $z+a_{l} x^{l}$ se $l \geq(2 k+3)$. 
Então para $h=z+a_{l} x^{l}+b_{l-1} x^{l-1} y$,

$$
\begin{aligned}
& \eta_{1} h=2 l a_{l} x^{l}+2(l+k) b_{l-1} x^{l-1} y+3(k+1) z \\
& \eta_{2} h=2 b_{l-1} x^{l-1} z+x^{2 k+2}+4 x^{k+1} y+3 y^{2} \\
& \eta_{3} h=l a_{l} x^{l+k}+3 l a_{l} x^{l-1} y+(l-2 k-3) b_{l-1} x^{l+k-1} y+(3 l-3) b_{l-1} x^{l-2} y^{2} \\
& \eta_{4} h=l a_{l} x^{l-1} z+(l-1) b_{l-1} x^{l-2} y z+(k+1) x^{2 k+1} y+(k+1) x^{k} y^{2}
\end{aligned}
$$

assim, para $a_{l} \neq 0, x^{l-1} y \in T \mathcal{R}_{V}(h)$, segue-se pelo Lema de Mather que $h$ se reduz a $z+a_{l} x^{l}$.

Seja $h=z+a_{l} x^{l}$. Então

$$
\begin{aligned}
& \eta_{1} h=2 l a_{l} x^{l}+3(k+1) z \\
& \eta_{2} h=x^{2 k+2}+4 x^{k+1} y+3 y^{2} \\
& \eta_{3} h=l a_{l} x^{l+k}+3 l a_{l} x^{l-1} y \\
& \eta_{4} h=l a_{l} x^{l-1} z+(k+1) x^{2 k+1} y+(k+1) x^{k} y^{2}
\end{aligned}
$$

Pelo critério geométrico (é possível ter solução não trivial se $2 l=3 k+3$, ou seja, para $k$ impar ), para $a_{l} \neq 0$ e $a_{3(k+1) / 2}^{2} \neq-4 / 27, z+a_{l} x^{l}$ é finitamente determinado e uma forma pré-normal é dada por $z+a_{l} x^{l}+s_{l}(x)$.

Para $a_{l}=0$, seja $h=z+b_{l-1} x^{l-1} y \operatorname{com} l \leq(2 k+1)$ ent ao

$$
\begin{aligned}
& \eta_{1} h=2(l+k) b_{l-1} x^{l-1} y+3(k+1) z \\
& \eta_{2} h=2 b_{l-1} x^{l-1} z+x^{2 k+2}+4 x^{k+1} y+3 y^{2} \\
& \eta_{3} h=(l-2 k-3) b_{l-1} x^{l+k-1} y+(3 l-3) b_{l-1} x^{l-2} y^{2} \\
& \eta_{4} h=(l-1) b_{l-1} x^{l-2} y z+(k+1) x^{2 k+1} y+(k+1) x^{k} y^{2}
\end{aligned}
$$

Pelo critério geométrico (é possível ter solução não trivial se $2 l=k+3$, ou seja, para $k$ impar ), para $b_{l-1} \neq 0$ e $b_{\frac{(k+3)}{2}-1} \neq \pm 2, z+b_{l-1} x^{l-1} y$ é finitamente determinado e uma forma pré-normal é dada por $z+b_{l-1} x^{l-1} y+r(x, y)$

No teorema abaixo obtemos a classificação das seções de $S_{k}$ com relação a $\mathcal{K}_{R V^{-}}$ equivalência. Neste caso vários dos parâmetros modais do Teorema anterior são eliminados.

Teorema 3.6 Seja $V$ a imagem de $S_{k}=\left\{\left(X, Y^{2}, Y^{3}+X^{k+1} Y\right)\right\}, k \geq 1$. Todo germe 
de submersão $h:\left(\mathbb{C}^{3}, 0\right) \rightarrow(\mathbb{C}, 0)$ é $\mathcal{K}_{R V}$-equivalente a um dos seguintes germes

$$
\begin{array}{lll}
S_{k, 0} & x & \\
S_{k, 2} & y+x^{2} & \\
S_{k, 3} & y+x^{3} & \\
S_{k, 4} & y+x^{4} & \\
\vdots & \vdots & a_{k+1} \neq 0 \text { e } a_{k+1} \neq 1 \\
S_{k, k+1} & y+a_{k+1} x^{k+1} & a_{k+1} \neq 0, a_{k+1} \neq 1 \text { e }(k+2) \leq l \leq 2 k \\
S_{k, k+1}^{l} & y+a_{k+1} x^{k+1}+x^{l} & l \geq k+2 \\
S_{k, k+1,1}^{l} & y+x^{k+1}+x^{l} & l \geq k+2 \\
S_{k, l} & y+x^{l} & l \geq 2 \\
E_{l} & z+x^{l} & \text { se ké impare } l=3(k+1) / 2, \text { então } a_{l}^{2} \neq-4 / 27 \\
E_{l}(s) & z+a_{l} x^{l}+s(x) & 2 \leq l \leq 2 k+1 \\
E_{l}^{1}(r) & z+x^{l-1} y+r(x) & \text { se } k \text { é impar e } l=(k+3) / 2, \text { então } b_{l-1} \neq \pm 2 \\
E_{l}^{*}(r) & z+b_{l-1} x^{l-1} y+r(x, y)
\end{array}
$$

Prova: Análoga a prova do teorema anterior, observando que neste caso o espaço tangente a $h$ é o ideal gerado por $\eta_{1} h, \eta_{2} h, \eta_{3} h, \eta_{4} h$ e $h$. 


\section{Capítulo 4}

\section{Trivialidade Topológica de Família de Seções de Hipersuperfícies}

Este é o capítulo central do trabalho. Nele, propomos condiçōes suficientes para a trivialidade topológica de famílias de seções com métodos baseados no fecho integral do espaço tangente do grupo $\mathcal{R}_{V}$, estendendo parcialmente resultados de Teissier [38] e Gaffney [10].

Os métodos apresentados são utilizados para a obtenção de resultados precisos quando a variedade analítica é quase homogênea e a família de seções é uma deformação de um germe quase-homogêneo $f$ consistente $\operatorname{com} V$, por termos de filtração maior ou igual a filtração de $f$. Resultados de M. Saia [35] sobre a determinação do fecho integral de um ideal através de seu poliedro de Newton são usados para descrever um método eficiente para garantir a trivialidade topológica de famílias Newton não-degeneradas.

\subsection{Fatos básicos sobre fecho integral de ideais}

Definição 4.1 Seja I ideal de um anel $A$. Um elemento $h \in A$ pertence ao fecho integral de $I$, denotado por $\bar{I}$, se existe um polinômio mônico $P(z)=z^{n}+\sum_{i=0}^{n-1} a_{i} z^{n-i}, a_{i} \in$ $I^{i}$ tal que $P(h)=0$.

Quando $A=\mathcal{O}_{X, x_{0}}$, o anel local de um conjunto analítico complexo, Tessier [39] mostrou o seguinte: 
Teorema 4.1 Seja $X$ um espaço analítico complexo e $I$ um ideal em $\mathcal{O}_{X, x_{0}}$. Então as seguintes afirmações são equivalentes:

(a) $h \in \bar{I}$

(b) Para cada escolha de geradores $\left\{g_{i}\right\}$ de $I$ existe uma vizinhança $U$ de $x_{0}$ e uma constante $C>0$ tal que para todo $x \in U$, temos

$$
|h(x)| \leq C \sup _{i}\left|g_{i}(x)\right|
$$

(c) Para cada $\varphi:(\mathbb{C}, 0) \rightarrow\left(X, x_{0}\right)$, h० $\varphi$ pertence a $\left(\varphi^{*}(I)\right) \mathcal{O}_{1}$.

(d) Existe um $\mathcal{O}_{X, x_{0}}$ módulo fiel de tipo finito $L$ tal que h.L $\subset$ I.L.

Para o caso analítico real a definição mais adequada para o fecho integral é dada pela condição $(c)$. Mais precisamente,

Definição 4.2 Seja $I$ um ideal de $\mathcal{A}_{X, x_{0}}$, onde $X$ é um conjunto analítico real. Então o fecho integral real de $I$ é o conjunto dos $h$ tais que para toda função analítica real

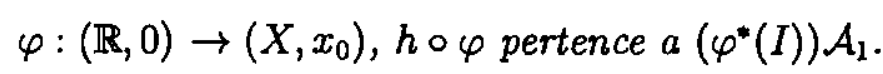

Gaffney em [10] pag. 30 mostra que $h \in \bar{I}$ se, e somente se, para cada escolha de geradores $\left\{g_{i}\right\}$ de $I$, existem uma vizinhança $U$ de $x_{0}$ e uma constante $C>0$ tal que

$$
|h(x)| \leq C s u p_{i}\left|g_{i}(x)\right| \text { para todo } x \in U
$$

\subsection{O Teorema Principal}

Seja $(V, 0) \subset\left(K^{n}, 0\right)$ um germe de variedade analítica e $I \subset K_{n}$ um ideal definindo $V$.

Dizemos que uma deformação $h:\left(K^{n} \times K^{r}, 0\right) \rightarrow(K, 0)$ de $h_{0}:\left(K^{n}, 0\right) \rightarrow(K, 0)$ é topologicamente $\mathcal{R}_{V}$-trivial ou $C^{0}-\mathcal{R}_{V}$-trivial, se existe homeomorfismo $\phi:\left(K^{n} \times\right.$ $\left.K^{r}, 0\right) \rightarrow\left(K^{n} \times K^{r}, 0\right)$ da forma $\phi(x, t)=(\bar{\phi}(x, t), t) \operatorname{com} \bar{\phi}(x, 0)=x, h \circ \phi(x, t)=h_{0}(x)$ e $\phi(V \times\{t\})=V \times\{t\}$.

A próxima proposição nos diz que o critério geométrico para a $\mathcal{R}_{V}$-determinação finita é uma propriedade aberta.

Proposição 4.1 Seja $h_{0}:\left(K^{n}, 0\right) \rightarrow(K, 0)$ um germe $\mathcal{R}_{V}$-finitamente determinado $e$ $h:\left(K^{n} \times K, 0\right) \rightarrow(K, 0)$ uma deformação de $h_{0}$. Então o germe $\left\{x \in K^{n}: d h_{t} \xi(x)=\right.$ $\left.0, \forall \xi \in \Theta_{V}\right\}$ em 0 é $\{0\}$ ou vazio para valores suficientemente pequenos dos parâmetros. 


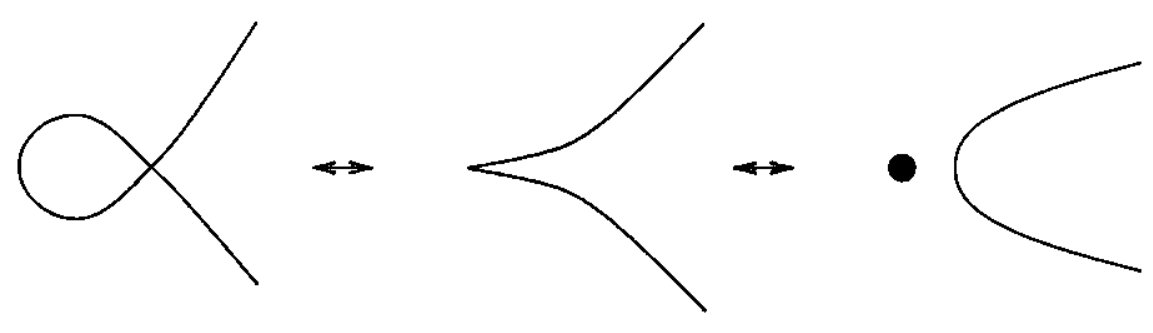

Figura 4.1: Deformação da cúspide.

A demonstração para o caso $K=\mathbb{R}$ segue igualmente ao caso $K=\mathbb{C}$ quando se considera a complexificação de $h_{0}$. A prova pode ser encontrada em [4].

Observemos que a proposição acima não garante a existência de uma vizinhança uniforme, ou seja, de uma vizinhança $U$ de $0 \mathrm{em}\left(K^{n}, 0\right)$ e uma bola aberta, $B_{\varepsilon}$, de centro na origem e raio $\varepsilon$ de $K$ tal que a condição acima se verifica. Então precisamos definir o seguinte:

Definição 4.3 Seja $h_{0}:\left(K^{n}, 0\right) \rightarrow(K, 0)$ um germe $\mathcal{R}_{V}$-finitamente determinado. Dizemos que uma deformação $h:\left(K^{n} \times K, 0\right) \rightarrow(K, 0)$ de $h_{0}$ é uma boa deformação se existe vizinhança $U$ de $0 \mathrm{em}\left(K^{n}, 0\right)$ e $\varepsilon>0$ tal que $h_{t}-\mathcal{R}_{V}$-finitamente determinado (então $h_{t}$ é transversal a $V$ fora de 0 ) para todo $x \in U$ et $t \in B_{\varepsilon}$, onde $B_{\varepsilon}$ é a bola aberta de centro na origem e raio $\varepsilon$ de $K$.

Exemplo 4.1 Seja $V$ o eixo-x em $K^{2}, \Theta_{V}$ é gerado por $(1,0) e(0, y)$. Temos que $h_{0}(x, y)=x^{2}+y^{3}$ é $\mathcal{R}_{V}$-finito. Considere a deformação $h_{t}(x, y)=x^{2}+y^{3}+t y^{2}$ de $h_{0}, h_{t}$ é $\mathcal{R}_{V}$-finito para cada $t$ fixo, mas não existe $\varepsilon>0$ tal que $h_{t}$ é $\mathcal{R}_{V}$-finito para $t \in B_{\varepsilon}$, ou seja, $h$ não é uma boa deformação de $h_{0}$ (ver Figura 4.1).

Podemos supor na sequência que $d h_{t} \xi(0)=0, \forall \xi \in \Theta_{V}$. De fato, se $d h_{t} \xi(0) \neq 0$ para algum $\xi \in \Theta_{V}$, então $d h_{t} \xi \cdot \frac{\partial h}{\partial t}=d h_{t}\left(\frac{\partial h}{\partial t} \cdot \xi\right)$

Assim,

$$
\frac{\partial h}{\partial t}=d h_{t}\left(\frac{\frac{\partial h}{\partial t} \cdot \xi}{d h_{t} \xi}\right)
$$

portanto, pela Proposição $3.2, h$ é uma deformação $C^{\omega}$ - $\mathcal{R}_{V}$-trivial (analítica trivial).

Observemos que podemos ter $d h_{t} \xi(0)=0 \operatorname{com} \xi(0) \neq 0$. 
Lema 4.1 Sejam $I$ e $J$ ideais de $\mathcal{O}_{n}$ (ou $\mathcal{A}_{n}$ ) com $\mathcal{M}_{n} I \subseteq J \subseteq I$ e $\mathcal{V}(I)=\{0\}$, onde $\mathcal{V}(I)$ é a variedade do ideal $I$. Então $\mathcal{V}(J)=\{0\}$,.

Prova : Da hipótese temos que

$$
\mathcal{V}\left(\mathcal{M}_{\mathrm{n}} I\right) \supseteq \mathcal{V}(J) \supseteq \mathcal{V}(I)
$$

como $\mathcal{V}\left(\mathcal{M}_{n} I\right)=\mathcal{V}\left(\mathcal{M}_{n}\right) \cup \mathcal{V}(I)=\{0\} \cup\{0\}$ segue-se que $\mathcal{V}(J)=\{0\}$

Sejam $h_{0}:\left(K^{n}, 0\right) \rightarrow(K, 0)$ um germe $\mathcal{R}_{V}$-finitamente determinado, $h:\left(K^{n} \times K, 0\right) \rightarrow(K, 0)$ uma deformação de $h_{0},\left\{\xi_{1}, \ldots, \xi_{r}\right\}$ geradores de $\Theta_{V} \mathrm{e}$ $s_{i}=d h_{t} \xi_{i}$. Se denotarmos por $I$ o ideal de $K_{n+1}$ gerado por $\left\{s_{1}, \ldots, s_{r}\right\}$ então segue da Proposição 4.1 que $\mathcal{V}(I)=\{0\} \times K$. Sejam $\left\{\alpha_{1}, \ldots, \alpha_{m}\right\}$ geradores de $\Theta_{V}^{0}$, tomando-se $d h_{t} \alpha_{i}=\rho_{i}$ e $J$ o ideal gerado por $\left\{\rho_{1}, \ldots, \rho_{m}\right\}$, vem que $\mathcal{M}_{n} I \subset J \subset I$ e pelo Lema 4.1 temos que $\mathcal{V}(J)=\{0\} \times K$.

Seja $\rho(x, t)=\sum_{i=1}^{m}\left|\rho_{i}\right|^{2}$. Da condição $\mathcal{V}(J)=\{0\} \times K$ segue-se que $\rho \geq 0 \mathrm{e}$ $\rho_{t}(x)=0 \Leftrightarrow x=0$. Com isto provamos o seguinte Lema:

Lema 4.2 Como acima, sejam $h_{0}$ um germe $\mathcal{R}_{V}$-finitamente determinado $e$ $h:\left(K^{n} \times K, 0\right) \rightarrow(K, 0)$ uma boa deformaçãa de $h_{0}$. Se $\rho(x, t)=\sum_{i=1}^{m}\left|d h_{t} \alpha_{i}\right|^{2}$, então $\mathcal{V}(\rho(x, t))=\{0\} \times K$.

O resultado principal deste capítulo é o seguinte teorema:

Teorema 4.2 Sejam $h_{0}:\left(K^{n}, 0\right) \rightarrow(K, 0)$ um germe $\mathcal{R}_{V}$-finitamente determinado $e$ $h:\left(K^{n} \times K, 0\right) \rightarrow(K, 0)$ uma boa deformação de $h_{0}$. Se $\frac{\partial h}{\partial t} \in \overline{d h_{t}\left(\Theta_{V}^{0}\right)}$, então $h e ́$ $C^{0} \mathcal{R}_{V}$-trivial.

Prova: Com as notações acima vem que

$$
\left|\rho_{i}\right|^{2} \frac{\partial h}{\partial t}=d h_{t}\left(\overline{\rho_{i}} \frac{\partial h}{\partial t} \alpha_{i}\right)
$$

Sendo $\rho=\sum_{i=1}^{m}\left|\rho_{i}\right|^{2}$, temos

$$
\rho \frac{\partial h}{\partial t}=d h_{t}\left(\frac{\partial h}{\partial t}\left(\overline{\rho_{1}} \alpha_{1}+\ldots+\overline{\rho_{m}} \alpha_{m}\right)\right)
$$


então

$$
\frac{\partial h}{\partial t}=d h_{t}\left(\frac{\partial h}{\partial t} \frac{1}{\rho}\left(\overline{\rho_{1}} \alpha_{1}+\ldots+\overline{\rho_{m}} \alpha_{m}\right)\right)
$$

Pelo Lema $4.2 \mathcal{V}(\rho(x, t))=\{0\} \times K$. Então podemos definir o campo de vetores $X$ em $\left(K^{n} \times K, 0\right)$,

$$
X(x, t)= \begin{cases}\left(\frac{\partial h}{\partial t} \frac{1}{\rho}\left(\overline{\rho_{1}} \alpha_{1}+\ldots+\overline{\rho_{m}} \alpha_{m}\right), 1\right) & \text { se } x \neq 0 \\ (0,1) & \text { se } x=0\end{cases}
$$

O campo $X(x, t)$ é analítico real fora de $\{0\} \times K$. Mostraremos que $X$ satisfaz uma condição de Lipschitz ao longo da solução $(0, t)$, o que garante a continuidade do campo $X$ e mais ainda a sua integrabilidade ( unicidade de solução ) na vizinhança de $(0,0)$.

Por hipótese $\frac{\partial h}{\partial t} \in \overline{d h_{t}\left(\Theta_{V}^{0}\right)}$ e portanto pelo item b) do Teorema 4.1

$$
\left|\frac{\partial h}{\partial t}\right| \leq \operatorname{csup}\left\{\left|\rho_{i}\right|\right\}
$$

Portanto

$$
\begin{aligned}
|X(x, t)-X(0, t)| & =\left|\frac{\partial h}{\partial t} \frac{1}{\rho}\left(\overline{\rho_{1}} \alpha_{1}+\ldots+\overline{\rho_{m}} \alpha_{m}\right)\right| \\
& \leq\left|\frac{\partial h}{\partial t}\right| \frac{1}{\rho}\left(\left|\overline{\rho_{1}}\right|\left|\alpha_{1}\right|+\ldots+\left|\overline{\rho_{m}}\right|\left|\alpha_{m}\right|\right) \\
& \leq \operatorname{csup}\left\{\left|\rho_{i}\right|\right\} \frac{1}{\rho}\left(\left|\rho_{1}\right|\left|\alpha_{1}\right|+\ldots+\left|\rho_{m}\right|\left|\alpha_{m}\right|\right) \\
& \leq \operatorname{csup}\left\{\left|\rho_{i}\right|\right\} \frac{1}{\sqrt{\rho} \sqrt{\rho}}\left(\sqrt{\rho}\left|\alpha_{1}\right|+\ldots+\sqrt{\rho}\left|\alpha_{m}\right|\right) \\
& \leq c\left(\left|\alpha_{1}\right|+\ldots+\left|\alpha_{m}\right|\right) \leq C|x|
\end{aligned}
$$

Assim, $X$ satisfaz uma condição de Lipschitz ao longo da solução $(0, t)$, então por [24] ou por [2] $X(x, t)$ é localmente integrável na vizinhança de $(0,0) \in K^{n} \times K$, isto é, podemos encontrar uma família de homeomorfimos $\phi(x, t, \tau), \phi:\left(\left(K^{n} \times K\right) \times \mathbb{R}, 0\right) \rightarrow$ $\left(K^{n} \times K, 0\right)$ tais que $\frac{\partial \phi}{\partial \tau}=-X \circ \phi$ e $\phi(x, t, 0)=(x, t)$. Para finalizar a prova precisamos do seguinte lema.

Lema 4.3 Seja $h:\left(K^{n} \times K, 0\right) \rightarrow(K, 0)$ uma deformação de $h_{0}$. Suponha que existe campo de vetores $(W, 1) \in \operatorname{Derlog}(V \times K)$ tal que: 
(i) $\rho \frac{\partial h}{\partial t}=d h_{t}(W)$, onde $\rho$ é uma função controle, isto é, $\rho:\left(K^{n} \times K, 0\right) \rightarrow \mathbb{R}$ com $\rho(x, t) \geq 0$ e $\rho(x, t)=0$ se, e somente se, $x=0$.

(ii) $\left(\frac{W}{\rho}, 1\right)$ é localmente integrável.

Então h é topologicamente $\mathcal{R}_{V}$-trivial.

Prova: Seja $\phi(x, t, \tau)$ família de homeomorfismos tal que $\frac{\partial \phi}{\partial \tau}=-\left(\frac{W}{\rho}, 1\right) \circ \phi, \phi(x, t, 0)=$ $(x, t)$. Então definimos, no caso $K=\mathbb{R}$,

$$
\varphi(x, t)=\phi(x, 0, t), \text { por (iii) temos que } \varphi(x, t)=(\bar{\varphi}(x, t), t) .
$$

Derivando $h(\varphi(x, t))=h(\bar{\varphi}(x, t), t)$ com relação a $t$ temos

$$
\begin{aligned}
\frac{\partial}{\partial t}(h(\varphi(x, t))) & =\sum_{i=1}^{n} \frac{\partial h}{\partial x_{i}}(\bar{\varphi}(x, t), t) \frac{\partial \bar{\varphi}_{i}}{\partial t}(x, t)+\frac{\partial h}{\partial t}(\bar{\varphi}(x, t), t) \\
& =-\sum_{i=1}^{n} \frac{\partial h}{\partial x_{i}}(\bar{\varphi}(x, t), t) \frac{W_{i}}{\rho}(\bar{\varphi}(x, t), t)+\frac{\partial h}{\partial t}(\bar{\varphi}(x, t), t) \\
& =\left(\frac{\partial h}{\partial t}-\sum_{i=1}^{n} \frac{W_{i}}{\rho} \frac{\partial h}{\partial x_{i}}\right)(\bar{\varphi}(x, t), t)=0
\end{aligned}
$$

onde $W_{i}$ são as componentes de $W$. Portanto, fixando $x$, temos que $h(\varphi(x, t))$ é constante, isto é, $h(\varphi(x, t))=h(\varphi(x, 0))=h(x, 0)=h_{0}(x)$ para todo $t$ e $x$. Portanto $h$ é topologicamente $\mathcal{R}_{V}$-trivial. Para $K=\mathbb{C}$, considere a restrição

$$
h^{1}=h \mid \mathbb{C}^{n} \times \mathbb{R} \times\{0\} \rightarrow \mathbb{C} .
$$

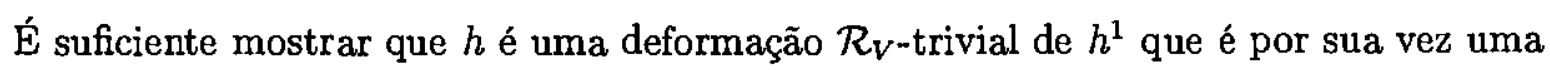
deformação topologicamente $\mathcal{R}_{V}$-trivial de $h_{0}$.

Seja $\phi(x, t, \tau)$ tal que $\frac{\partial \phi}{\partial \tau}=-\left(\frac{W}{\rho}, 1\right) \circ \phi$ e $\phi(x, t, 0)=(x, t)$. Considere $\varphi_{1}(x, u+i v)=$ $\phi(x, u, v)$ e $\varphi_{2}(x, u)=\phi(x, 0, u)$. Mostra-se que $h \circ \phi_{1}$ é constante em relação a $v$ e portanto $h(\phi(x, u+i v))=h\left(\phi_{\mathrm{I}}(x, u)\right)=h(\varphi(x, u, 0))=h(x, u)=h^{1}(x, u)$. Também mostra-se que $h^{1} \circ \phi_{2}$ é constante em relação a $u$, então $h^{1}\left(\phi_{2}(x, u)\right)=h^{1}\left(\phi_{2}(x, 0)\right)=$ $h^{1}(x, 0)=h_{0}$ e o resultado segue.

\subsection{O Caso Quase-Homogêneo}

Definição 4.4 (a) Dados $\left(w_{1}, \ldots, w_{n}: d_{1}, \ldots, d_{p}\right), w_{i}, d_{j} \in \mathcal{Q}^{+}$, um germe de aplicação $f:\left(K^{n}, 0\right) \rightarrow\left(K^{p}, 0\right)$, é quase-homogêneo de tipo $\left(w_{1}, \ldots, w_{n}: d_{1}, \ldots, d_{p}\right)$ se para todo $\lambda \in K-\{0\}:$

$$
f\left(\lambda^{w_{1}} x_{1}, \lambda^{w_{2}} x_{2}, \ldots, \lambda^{w_{n}} x_{n}\right)=\left(\lambda^{d_{1}} f_{1}(x), \lambda^{d_{2}} f_{2}(x), \ldots, \lambda^{d_{p}} f_{p}(x)\right)
$$


Neste caso, chamamos ao valor $w_{i}$ de peso da variável $x_{i}$ e ao valor $d_{i}$, de filtração de $f_{i}$ em relação aos pesos $\left(w_{1}, \ldots, w_{n}\right)$. Notação: $\operatorname{peso}\left(x_{i}\right)=w\left(x_{i}\right)=w_{i}$ e filtração $(f)=$ $f i l(f)=\left(d_{1}, \ldots, d_{p}\right)$.

(b) Dados $\left(w_{1}, \ldots, w_{n}\right)$, para todo monômio $x^{\alpha}=x_{1}^{\alpha_{1}} x_{2}^{\alpha_{2}} \ldots x_{n}^{\alpha_{n}}$, definimos $f i l\left(x^{\alpha}\right)=$ $\sum_{i=1}^{n} \alpha_{i} w_{i}$.

(c) Define-se a filtração em $K_{n}$ via a função definida por $f i l(f)=i n f_{\alpha}\left\{f i l\left(x^{\alpha}\right)\right.$ : $\left.\frac{\partial^{\alpha} f}{\partial x^{\alpha}}(0) \neq 0\right\}$.

Definiçāo 4.5 Seja $h:\left(K^{n}, 0\right) \rightarrow(K, 0)$ um germe quase-homogêneo do tipo $\left(w_{1}, \ldots, w_{n} ; k\right)$. Definimos a função controle padrão de grau $r$ associada a $h$ por $p(x)=$ $\left|x_{1}\right|^{2 \alpha_{1}}+\left|x_{2}\right|^{2 \alpha_{2}}+\ldots+\left|x_{n}\right|^{2 \alpha_{n}}$ onde os $\alpha_{i}$ são escolhidos de forma que $p(x)$ seja quasehomogêneo de tipo $\left(w_{1}, \ldots, w_{n} ; 2 r\right)$.

Lema 4.4 Seja $h_{0}:\left(K^{n}, 0\right) \rightarrow(K, 0)$ um germe quase-homogêneo do tipo $\left(w_{1}, \ldots, w_{n} ; k\right)$ $e h_{t}, t \in B_{\varepsilon}$, onde $B_{\varepsilon}$ é a bola fechada de centro em 0 e raio $\varepsilon$ de $K$, uma deformação de $h_{0}$, a qual é quase-homogênea do mesmo tipo que $h_{0}$ e $p(x)$ é o controle padrão de grau $r$ associado a $h_{0}$. Então:

a) Existe constante $c$ tal que $\left|h_{t}(x)\right| \leq c p(x)^{k / 2 r}$;

b) Se existem constantes $\alpha$ e c tais que $\left|h_{t}(x)\right| \geq c|x|^{\alpha}$, então

$\left|h_{t}(x)\right| \geq c_{1} p(x)^{k / 2 r}$ para alguma constante $c_{1}$.

Prova: a) Seja $M=\left\{(y, t) \in K^{n} \times B_{\varepsilon}\right.$ tal que $\left.p(y)=1\right\}$

Para cada $(x, t), x \neq 0$ fixado, existe $(y, t) \in M$, e $\lambda \neq 0$ tal que

$$
(x, t)=\left(\lambda^{w_{1}} y_{1}, \ldots, \lambda^{w_{n}} y_{n}, t\right)
$$

Agora, seja $\beta=\sup \left\{\left|h_{t}(y)\right|\right.$ tal que $\left.(y, t) \in M\right\}$. Então

$$
\begin{aligned}
\left|h_{t}(x)\right|^{2 r / k} & =\left|h_{t}\left(\lambda^{w_{1}} y_{1}, \ldots, \lambda^{w_{n}} y_{n}\right)\right|^{2 r / k}=\left|\lambda^{k} h_{t}(y)\right|^{2 r / k} \\
& \leq\left|\lambda^{k} \beta\right|^{2 r / k}=\lambda^{2 r} \beta^{2 r / k}=\beta^{2 r / k} \lambda^{2 r} p(y)=\beta^{2 r / k} p(x)
\end{aligned}
$$

e segue o resultado.

Para provar b), seja $\delta=\inf \left\{\left|h_{t}(y)\right|\right.$ tal que $\left.(y, t) \in M\right\}$. Da hipotese, $\delta>0$, onde

$$
\left|h_{t}(x)\right|^{2 r / k}=\left|\lambda^{k} h_{t}(y)\right|^{2 r / k} \geq \lambda^{2 r} \delta^{2 r / k}=\delta^{2 r / k} \lambda^{2 r} p(y)=\delta^{2 r / k} p(x)
$$

donde segue o resultado. 
Observação 4.1 Observemos que, com as mesmas hipóteses do Lema 4.4, se $f i l(f(x))>f i l\left(h_{0}\right)$ então $|f(x)| / p(x)^{k / 2 r} \leq c \varphi(x)$ onde $\varphi(0)=0$ e cé constante.

Como um corolário da prova do Teorema 4.2 e usando a observação 4.1 temos o seguinte resultado.

Teorema 4.3 Sejam $h_{0}:\left(K^{n}, 0\right) \rightarrow(K, 0)$ um germe quase-homogêneo $\mathcal{R}_{V}$-finitamente determinado, $h:\left(K^{n} \times K, 0\right) \rightarrow(K, 0)$ deformação de $h_{0}, h_{t}(x)=h(x, t)$ com $f i l\left(h_{t}-h_{0}\right) \geq f i l\left(h_{0}\right)$ e $\rho(x, t) \geq \alpha p(x)$, onde $p(x)$ é a função controle padrão de grau $k$ associada a $h_{0}$. Então $h$ é $C^{0}-\mathcal{R}_{V}$-trivial.

Prova: Seguindo a demonstração do Teorema 4.2 temos que

$$
\begin{aligned}
|X(x, t)-X(0, t)| & \leq\left|\frac{\partial h}{\partial t}\right| \frac{1}{\rho}\left(\left|\overline{\rho_{1}}\right|\left|\alpha_{1}\right|+\ldots+\left|\overline{\rho_{m}}\right|\left|\alpha_{m}\right|\right) \\
& \leq\left|\frac{\partial h}{\partial t}\right| \frac{1}{\sqrt{\rho} \sqrt{\rho}}\left(\sqrt{\rho}\left|\alpha_{1}\right|+\ldots+\sqrt{\rho}\left|\alpha_{m}\right|\right) \\
& =\left|\frac{\partial h}{\partial t}\right| \frac{1}{\sqrt{\rho}}\left(\left|\alpha_{1}\right|+\ldots+\left|\alpha_{m}\right|\right) \\
& \leq\left|\frac{\partial h}{\partial t}\right| \frac{1}{\sqrt{\alpha p}}\left(\left|\alpha_{1}\right|+\ldots+\left|\alpha_{m}\right|\right) \leq C|x|
\end{aligned}
$$

e a conclusão é igual a do Teorema 4.2. Portanto $h$ é $C^{0}-\mathcal{R}_{V}$-trivial.

Embora o Teorema 4.3 estabeleça um resultado de $C^{0}-\mathcal{R}_{V}$-trivialidade para germes quase-homogêneos, sem hipóteses sobre $V$, a verificação da condição $\rho(x, t) \geq \alpha p(x)$ não é simples, o que limita sua aplicabilidade. Para resultados de aplicação mais direta será necessário assumir que $V$ e $h$ são ambos quase-homogêneos, para uma mesma escolha de pesos.

Definição 4.6 Um germe de variedade $(V, 0) \subseteq\left(K^{n}, 0\right)$ é quase-homogêneo se ele é definido por um germe de aplicação $f:\left(K^{n}, 0\right) \rightarrow\left(K^{p}, 0\right)$ que é quase-homogêneo.

Seguindo Damon em [6] temos a seguinte definição:

Definição 4.7 Seja $V$ definida por um polinômio quase-homogêneo. Dizemos que $h$ é consistente com $V$ se $h$ é quase-homogêneo com os pesos de $V$. 
Exemplo 4.2 Seja $V=\phi^{-1}(0) \subset K^{3}$ onde $\phi(x, y, z)=2 x^{2} y^{2}+y^{3}-z^{2}+x^{4} y$. Temos que $\phi$ é quase-homogêneo com pesos 1,2 e 3 para $x, y$ e $z$ respectivamente. Sejam $h(x, y, z)=x^{3}+x y+z$ e $f(x, y, z)=x^{3}+x y+z^{2}$. Segue-se que $h$ é consistente com $V, f$ é quase-homogêneo mas não consistente com $V$.

Suponhamos agora que $h_{0}:\left(K^{n}, 0\right) \rightarrow(K, 0)$ é $\mathcal{R}_{V}$-finitamente determinado consistente com $V$ e $d h_{0}\left(\alpha_{i}\right)$ quase-homogêneo para cada $i$, onde $\left\{\alpha_{i}\right\}$ são geradores de $\Theta_{V}^{0}$. Definimos

$$
\omega_{0}(x)=\left|d h_{0}\left(\alpha_{1}\right)(x)\right|^{2 s_{1}}+\ldots+\left|d h_{0}\left(\alpha_{r}\right)(x)\right|^{2 s_{r}}
$$

onde $s_{i}=k / r_{i}, r_{i}$ é a filtração de $d h_{0}\left(\alpha_{i}\right)$ e $k=m . m . c .\left\{r_{i}\right\}$ para $i=1, \ldots, m$. Como $h_{0}$ é $\mathcal{R}_{V}$-finito segue que $\omega_{0}(x)=0$ se, e somente se, $x=0$ e, portanto $\omega_{0}(x) \geq c|x|^{\alpha}$ para constantes $c$ e $\alpha$.

Para deformações $h_{t}$ de $h_{0} \operatorname{com} \operatorname{fil}\left(h_{t}\right) \geq \operatorname{fil}\left(h_{0}\right)$ definimos $\omega(x, t)=\sum_{i=1}^{m}\left|\rho_{i}\right|^{2 s_{i}}$, sendo $\rho_{i}=d h_{t}\left(\alpha_{i}\right)$. Assim, temos que fil $(\omega) \geq \operatorname{fil}\left(\omega_{0}\right)$.

Lema 4.5 Existe constante $c$ tal que $\omega(x, t) \geq c p(x)$, sendo $p(x)$ a função controle padrão de grau $k$ associada $a \omega_{0}$.

Prova: Quando $h_{t}$ é quase-homogêneo de mesmo tipo que $h_{0}$, então $\omega_{0}$ e $\omega$ são quasehomogêneos do mesmo tipo e o resultado segue do Lema 4.4.

Se fil $\left(h_{t}-h_{0}\right)>\operatorname{fil}\left(h_{0}\right)$, escrevemos $\omega(x, t)=\omega_{0}(x)+R(x, t) \operatorname{com} \operatorname{fil}(R(x, t))>$ fil $\left(\omega_{0}(x)\right)=2 k$. Então $\omega_{0}(x) \leq \omega(x, t)+|R(x, t)|$. Pela parte b) do Lema 4.4 , existe constante $c_{1}$ tal que $c_{1} p(x) \leq \omega_{0}(x) \leq \omega(x, t)+|R(x, t)|$.

Desde que fil $(R(x, t))>\operatorname{fil}\left(\omega_{0}(x)\right)$, segue-se que $\lim _{x \rightarrow 0}|R(x, t)| / p(x)=0$ (Observação 4.1$)$. Então $\omega(x, t) \geq c p(x)$.

Uma consequência do Lema acima é que se $h_{t}$ é uma deformação por termos de filtração maior ou igual a filtração de $h_{0}$, com $h_{0} \mathcal{R}_{V}$-finito e quase-homogêneo então $h_{t}$ é uma boa deformação de $h_{0}$, para $t$ suficientemente pequeno.

Definição 4.8 Seja $(V, 0) \subseteq\left(K^{n}, 0\right)$ um germe de variedade analítica quase-homogêneo. Dizemos que um conjunto $\left\{\alpha_{1}, \ldots, \alpha_{r}\right\}$ de geradores de $\Theta_{V}$ é quase-homogêneo de tipo $\left(w_{1}, \ldots, w_{n}: d_{1}, \ldots, d_{r}\right)$ se $\alpha_{i}=\sum_{j=1}^{n} \alpha_{i j} \frac{\partial}{\partial x_{j}}, w\left(x_{j}\right)=w_{j}, w\left(\frac{\partial}{\partial x_{j}}\right)=-w_{j}$ ef fll $\left(\alpha_{i}\right)=d_{i}=$ $f i l\left(\alpha_{i j}\right)-w_{j}$ para cada $i=1$...r. 
Veremos no Teorema 4.4 que o conhecimento de um sistema de geradores quasehomogêneos para $\Theta_{V}$ é importante. Vimos na seção 3.4 que as singularidades $S_{k}$ são definidas por polinômios quase-homogêneos $\varphi_{k}(x, y, z)=2 x^{k+1} y^{2}+y^{3}-z^{2}+x^{2(k+1)} y=0$ e

$$
\begin{aligned}
& \eta_{1}=(2 x, 2(k+1) y, 3(k+1) z) \\
& \eta_{2}=\left(0,2 z, x^{2(k+1)}+4 x^{k+1} y+3 y^{2}\right) \\
& \eta_{3}=\left(x^{k+1}+3 y,-2(k+1) x^{k} y, 0\right) \\
& \eta_{4}=\left(z, 0,(k+1)\left(x^{2 k+1} y+x^{k} y^{2}\right)\right)
\end{aligned}
$$

são geradores quase-homogêneos de $\Theta_{V}$.

Uma outra classe de variedades quase-homogêneas para as quais é possivel exibir um sistema quase-homogêneo de geradores para o Derlog é a classe das interseções completas com singularidades isoladas. Mais precisamente,

Proposição 4.2 ([4], Proposição 7.2) Seja $(V, 0) \subseteq\left(\mathbb{C}^{n}, 0\right)$ um germe de interseção completa com singularidade isolada, definida por um germe de aplicação $f:\left(\mathbb{C}^{n}, 0\right) \rightarrow$ $\left(\mathbb{C}^{p}, 0\right)$ quase-homogêneo. Então $\Theta_{V}$ é gerado pelos campos de vetores $f_{i} \frac{\partial}{\partial x_{j}}$, onde $i=1, \ldots, p$ e $j=1, \ldots, n$, o campo de Euler $\sum_{i=1}^{n} w_{j} \frac{\partial}{\partial x_{i}}$, onde $w_{j}$ é o peso de $x_{j}$ para cada $j=1, \ldots n$ e os campos de vetores triviais:

$$
\left|\begin{array}{ccc}
\frac{\partial}{\partial x_{i_{1}}} & \cdots & \frac{\partial}{\partial x_{i_{p+1}}} \\
\frac{\partial f_{1}}{\partial x_{i_{1}}} & \cdots & \frac{\partial f_{1}}{\partial x_{i_{p+1}}} \\
\vdots & \vdots & \vdots \\
\frac{\partial f_{p}}{\partial x_{i_{1}}} & \cdots & \frac{\partial f_{p}}{\partial x_{i_{p+1}}}
\end{array}\right|
$$

para todas $(p+1)$-uplas $i_{1}, \ldots, i_{p+1}$ satisfazendo $1 \leq i_{1}<i_{2}<\ldots<i_{p+1} \leq n$.

Segue-se da Definição 4.8 que:

Lema 4.6 Se $V$ é quase homogêneo e $\left\{\alpha_{1}, \ldots, \alpha_{\tau}\right\}$ um conjunto de geradores quasehomogêneos de $\Theta_{V}$ com $f i l\left(\alpha_{i}\right)=d_{i}$ e $h_{0}:\left(K^{n}, 0\right) \rightarrow(K, 0)$ consistente com $V e$ $f i l\left(h_{0}\right)=d$ então $d h_{0}\left(\alpha_{i}\right)$ é quase-homogêneo de filtração $d-d_{i}$ para cada $i=1, \ldots, r$.

Prova: Temos que $d h_{0}\left(\alpha_{i}\right)=\sum_{j=1}^{n} \frac{\partial h_{0}}{\partial x_{j}} \alpha_{i j}$. Como $d=$ fil $\left(h_{0}\right)$, segue-se que fil $\left(\frac{\partial h_{0}}{\partial x_{j}} \alpha_{i j}\right)=$ $d-w_{j}+d_{i}+w_{j}=d-d_{i}$ para $\forall j=1, \ldots, n$, portanto fil $\left(d h_{0}\left(\alpha_{i}\right)\right)=d-d_{i}$, e segue o resultado. 
Teorema 4.4 Sejam $V$ uma variedade quase-homogênea, $\left\{\alpha_{i}\right\}$ um conjunto de geradores quase-homogêneos de $\Theta_{V}^{0}$ e $h_{0}:\left(K^{n}, 0\right) \rightarrow(K, 0)$ consistente com $V, \mathcal{R}_{V^{-}}$ finitamente determinado. Então toda deformação $h_{t}$ de $h_{0} \operatorname{com} f i l\left(h_{t}-h_{0}\right) \geq f i l\left(h_{0}\right) \dot{e}$ $C^{0}-\mathcal{R}_{V}$-trivial.

Prova: Pelo Lema 4.6, temos $d h_{0}\left(\alpha_{i}\right)$ é quase-homogêneo. Sejam $r_{i}$ a filtração de $d h_{0}\left(\alpha_{i}\right), i=1, \ldots, m \mathrm{e}$

$$
\omega_{0}(x)=\left|d h_{0}\left(\alpha_{1}\right)(x)\right|^{2 s_{1}}+\ldots+\left|d h_{0}\left(\alpha_{r}\right)(x)\right|^{2 s_{r}}
$$

onde $s_{i}=k / r_{i}$, e $k=m . m . c .\left\{r_{i}\right\}$.

Pondo $\rho_{i}=d h_{t}\left(\alpha_{i}\right)$ e $\omega=\sum_{i=1}^{m}\left|\rho_{i}\right|^{2 s_{i}}$. Temos que

$$
\left|\rho_{i}\right|^{2} \frac{\partial h}{\partial t}=d h_{t}\left(\overline{\rho_{i}} \frac{\partial h}{\partial t} \alpha_{i}\right)
$$

Portanto

$$
\omega \frac{\partial h}{\partial t}=d h_{t}\left(\frac{\partial h}{\partial t}\left(\overline{\rho_{1}}\left|\rho_{1}\right|^{2 s_{1}-2} \alpha_{1}+\ldots+\overline{\rho_{m}}\left|\rho_{m}\right|^{2 s_{m}-2} \alpha_{m}\right)\right)
$$

então

$$
\frac{\partial h}{\partial t}=d h_{t}\left(\frac{\partial h}{\partial t} \frac{1}{\omega}\left(\overline{\rho_{1}}\left|\rho_{1}\right|^{2 s_{1}-2} \alpha_{1}+\ldots+\overline{\rho_{m}}\left|\rho_{m}\right|^{2 s_{m}-2} \alpha_{m}\right)\right)
$$

Seja

$$
u(x, t)= \begin{cases}\left(\frac{\partial h}{\partial t} \frac{1}{\omega}\left(\overline{\rho_{1}}\left|\rho_{1}\right|^{2 s_{1}-2} \alpha_{1}+\ldots+\overline{\rho_{m}}\left|\rho_{m}\right|^{2 s_{m}-2} \alpha_{m}\right), 1\right) & \text { se } x \neq 0 \\ (0,1) & \text { se } x=0\end{cases}
$$

Portanto

$$
\begin{aligned}
|u(x, t)-u(0, t)| & \leq\left|\frac{\partial h}{\partial t}\right| \frac{1}{\omega}\left(\left|\overline{\rho_{1}}\right|\left|\rho_{1}\right|^{2 s_{1}-2}\left|\alpha_{1}\right|+\ldots+\left|\overline{\rho_{m}}\right|\left|\rho_{m}\right|^{2 s_{m}-2}\left|\alpha_{m}\right|\right) \\
& =\left|\frac{\partial h}{\partial t}\right| \frac{1}{\omega}\left(\left|\rho_{1}\right|^{2 s_{1}-1}\left|\alpha_{1}\right|+\ldots+\left|\rho_{m}\right|^{2 s_{m}-1}\left|\alpha_{m}\right|\right) \\
& =\frac{1}{\omega}\left(\left|\rho_{1}\right|^{2 s_{1}-1}\left|\frac{\partial h}{\partial t} \alpha_{1}\right|+\ldots+\left|\rho_{m}\right|^{2 s_{m}-1}\left|\frac{\partial h}{\partial t} \alpha_{m}\right|\right)
\end{aligned}
$$

Sendo fil $\left(d h_{0}\left(\alpha_{i}\right)\right)=r_{i}$, segue-se que $\left(f i l\left(h_{0}\right)-w_{j}\right)+$ fil $\left(\alpha_{i j}\right)=r_{i}$, com $w_{j}$ pesos das variáveis $x_{j}$ e $\alpha_{i j}$ a $j$-ésima componente de $\alpha_{i}$, o que implica em fil $\left(h_{0}\right)+f i l\left(\alpha_{i j}\right)>r_{i}$.

Denotando por $\left(\frac{\partial h}{\partial t} \alpha_{i}\right)_{j}$ a $j$-ésima componente de $\frac{\partial h}{\partial t} \alpha_{i}$, por hipótese vem que $\operatorname{fil}\left(\left(\frac{\partial h}{\partial t} \alpha_{i}\right)_{j}\right)>r_{i}$ 
Portanto, $|u(x, t)-u(0, t)| \leq C|x|$, o que implica em $u$ ser integrável. Portanto $h$ é $C^{0}-\mathcal{R}_{V}$-trivial.

Exemplo 4.3 Seja

$$
\varphi(x, y, z)=2 x^{k+1} y^{2}+y^{3}-z^{2}+x^{2(k+1)} y=0
$$

a equação para $V$ (equação reduzida para a imagem de $S_{k}$ ). Observemos que $\varphi$ é quase homogênea com pesos $2,2 k+2$ e $3 k+3$ para $x, y$ e $z$ respectivamente.Temos que $h(x, y, z)=y+a_{k+1} x^{k+1}$ é $\mathcal{R}_{V}$-finito para $a_{k+1} \neq 0,1$ e consistente com $V$. Portanto deformações de $h$ por termos de ordem maior ou igual a fil $(h)$ são $C^{0}-\mathcal{R}_{V}$-triviais. Para $k$ impar, $h_{1}(x, y, z)=z+a x^{3(k+1) / 2}$ e $h_{2}(x, y, z)=z+b x^{(k+1) / 2} y$ são consistentes com $V$ e $\mathcal{R}_{V}$-finitos para $a^{2} \neq-4 / 27$ e $b \neq \pm 2$. Portanto deformações de $h_{1}$ e $h_{2}$, respectivamente por termos de ordem maior ou igual a $\operatorname{fil}\left(h_{1}\right)$ e $\operatorname{fil}\left(h_{2}\right)$ são $C^{0}-\mathcal{R}_{V^{-}}$ triviais.

O exemplo seguinte mostra que a condição do Teorema 4.4 pode estar satisfeita sem que $\frac{\partial h}{\partial t} \in \overline{d h_{t}\left(\Theta_{V}\right)}$.

Exemplo 4.4 No exemplo acima tomando-se $k=1$ temos que $\Theta_{V}$ é gerado por $\alpha_{1}=$ $(2 x, 4 y, 6 z), \alpha_{2}=\left(0,2 z, x^{4}+4 x^{2} y+3 y^{2}\right), \alpha_{3}=\left(x^{2}+3 y,-4 x y, 0\right), \alpha_{4}=\left(z, 0,2 x^{3} y+2 x y^{2}\right)$. Deformações do germe $h_{0}(x, y, z)=y+a x^{2}, a \neq 0,1$ por termos de filtração maior ou igual a $f i l\left(h_{0}\right)=2$ são $\mathcal{R}_{V}$-topologicamente triviais, em particular $h(x, y, z, t)=$ $y+(a+t) x^{2}$ é $\mathcal{R}_{V}$-topologicamente trivial.

Entretanto, $\frac{\partial h}{\partial t}=x^{2}$ não pertence ao fecho integral do ideal $d h_{t}\left(\Theta_{V}^{0}\right)$. De fato, dado $\phi:(K, 0) \rightarrow\left(K^{4}, 0\right), \phi(s)=\left(s,-a s^{2}, 0,0\right)$ segue-se que $\frac{\partial h}{\partial t} \circ \phi$ não pertence a $\left(\phi^{*}\left(d h_{t}\left(\Theta_{V}^{0}\right)\right)\right) \mathcal{O}_{1}$ então, pelo Teorema 4.1 parte c), $\frac{\partial h}{\partial t}=x^{2} \notin \overline{d h_{t}\left(\Theta_{V}^{0}\right)}$.

\subsection{O Fecho Integral e o Poliedro de Newton}

A aplicabilidade do Teorema 4.2 está relacionada com a possibilidade de calcular o fecho integral do espaço tangente a $\mathcal{R}_{V}$-órbita.

M. Saia em [35] estende o conceito usual de poliedro de Newton de um germe [23], definindo o poliedro de Newton associado a um ideal de $K_{n}$, como segue. Para um 
sistema de coordenadas $x$ fixo em $K^{n}$ podemos identificar $K_{n}$ com o anel $K[[x]]$ das séries de potências convergentes. Para cada germe $g(x)=\sum a_{k} x^{k}$ define-se supp $g=$ $\left\{k \in \mathbb{Z}^{n}: a_{k} \neq 0\right\}$

Definição 4.9 (i) Seja I um ideal em $K_{n}$, define-se

$$
\text { supp } I=\cup\{\operatorname{supp} g: g \in I\}
$$

(ii) O poliedro de Newton de $I$, denotado por $\Gamma_{+}(I)$, é o fecho convexo em $\mathbb{R}_{+}^{n}$ do conjunto $\cup\left\{k+v: k \in \operatorname{supp} I, v \in \mathbb{R}_{+}^{n}\right\}$

(iii) A fronteira de Newton de $I$, denotada por $\Gamma(I)$, é a união das faces compactas de $\Gamma_{+}(I)$.

(iv) $I=\left\langle g_{1}, \ldots, g_{s}>\right.$ é Newton não degenerado se para cada face compacta $\triangle \subset$ $\Gamma(I)$, as equações $g_{1 \Delta}(x)=g_{2 \Delta}(x)=\ldots=g_{s \Delta}(x)=0$ não têm solução comum em $(K-\{0\})^{n}$, onde $g_{i \Delta}$ é a restrição de $g_{i}$ à face $\triangle$,ou seja, se $g_{i}(x)=\sum a_{k} x^{k}$ então $g_{i \Delta}(x)=\sum_{k \in \Delta} a_{k} x^{k}$.

Denote por $C(\bar{I})$ o fecho convexo em $\mathbb{R}_{+}^{n}$ do conjunto $\cup\left\{m: x^{m} \in \bar{I}\right\}$

O principal resultado em [35] é o seguinte teorema:

Teorema 4.5 Seja $I=<g_{1}, \ldots, g_{s}>$ um ideal de codimensão finita em $K_{n}$. Então $I$ é Newton não degenerado se, e somente se, $\Gamma_{+}(I)=C(\bar{I})$ (sempre vale $C(\bar{I}) \subseteq \Gamma_{+}(I)$ ).

Em nosso caso tomaremos $I=d h_{t}\left(\Theta_{V}^{0}\right)$ e $I_{0}=d h_{0}\left(\Theta_{V}^{0}\right)$. Os próximos resultados mostram que $C\left(\overline{I_{0}}\right)$ também dá uma condição para $C^{0}-\mathcal{R}_{V}$-trivialidade.

Lema 4.7 Seja $h(x, t)=h_{0}(x)+t g(x)$ uma deformação do germe $h_{0}$ com $g \in \overline{d h_{0}\left(\Theta_{V}^{0}\right)}$ e $d g\left(\alpha_{i}\right) \in \overline{d h_{0}\left(\Theta_{V}^{0}\right)}$, onde $\alpha_{i}$ são geradores de $\Theta_{V}^{0}$ então:

a) $h$ é uma boa deformação;

b) $g \in \overline{d h_{t}\left(\Theta_{V}^{0}\right)}$, para $t$ suficientemente pequeno.

Prova: Por hipótese temos que existe vizinhança $U$ de 0 em $K^{n}$ tal que

$$
|t|\left|d g\left(\alpha_{i}\right)\right| \leq|t| c s u p_{j}\left|d h_{0}\left(\alpha_{j}\right)\right|
$$

por outro lado,

$$
\sup _{j}\left|d h_{t}\left(\alpha_{j}\right)\right|=\sup _{j}\left|d h_{0}\left(\alpha_{j}\right)+t d g\left(\alpha_{j}\right)\right|
$$




$$
\begin{gathered}
\geq \sup _{j}\left|d h_{0}\left(\alpha_{j}\right)\right|-|t| \sup _{j}\left|d g\left(\alpha_{j}\right)\right| \\
\geq \sup _{j}\left|d h_{0}\left(\alpha_{j}\right)\right|-|t| \operatorname{csup}_{j}\left|d h_{0}\left(\alpha_{j}\right)\right| \geq(1-\alpha) \sup _{j}\left|d h_{0}\left(\alpha_{j}\right)\right|
\end{gathered}
$$

para algum $0<\alpha<1$ e $|t| \leq \frac{\alpha}{c}$.

Portanto, $\sup _{j}\left|d h_{t}\left(\alpha_{j}\right)\right| \geq C \sup _{j}\left|d h_{0}\left(\alpha_{j}\right)\right|$ para $t$ suficientemente pequeno e $C>0$. Desta desigualdade segue que

$$
\overline{d h_{0}\left(\Theta_{V}^{0}\right)} \subseteq \overline{d h_{t}\left(\Theta_{V}^{0}\right)}
$$

e temos $b$ ). Usando equivalência de normas vem que

$$
\rho(x, t) \geq c_{1} \sum_{j}\left|d h_{0}\left(\alpha_{j}\right)\right|^{2} \geq c_{2}|x|^{\alpha}
$$

para constantes $c_{1}, c_{2}$ e $\alpha$, onde $\rho(x, t)=\sum_{j}\left|d h_{t}\left(\alpha_{j}\right)\right|^{2}$, o que implica em $a$ ).

O exemplo abaixo mostra que $g \in \overline{d h_{0}\left(\Theta_{V}^{0}\right)}$ não implica que $d g\left(\alpha_{i}\right) \in \overline{d h_{0}\left(\Theta_{V}^{0}\right)}$.

Exemplo 4.5 Seja $V \subseteq K^{2}, 0$ definida por $\varphi(x, y)=x^{3}-y^{2}=0$. Temos que $\Theta_{V}$ é gerado por $\alpha_{1}=(2 x, 3 y), \alpha_{2}=\left(2 y, 3 x^{2}\right)$. Considere o germe $h_{0}(x, y)=x^{2}+y^{3}$. Assim, $I_{0}=<4 x^{2}+9 y^{3}, 4 x y+9 x^{2} y^{2}>$ e é não degenerado. Portanto, $C\left(\overline{I_{0}}\right)=\Gamma_{+}\left(I_{0}\right)$. Temos que $g=x y \in \overline{I_{0}}$ mas $d g\left(\alpha_{2}\right)=2 y^{2}+3 x^{3}$ não pertence a $\overline{I_{0}}$. De fato, dado $\phi: K, 0 \rightarrow K^{2}, 0, \phi(s)=(0, s)$ segue-se que $d g\left(\alpha_{2}\right) \circ \phi=2 s^{2}$ não pertence $\left(\phi^{*}\left(I_{0}\right)\right) \mathcal{O}_{1}$, então, pelo Teorema 4.1 parte $\mathrm{c}), d g\left(\alpha_{2}\right) \notin \overline{I_{0}}$.

Como consequência imediata do Lema 4.7 e do Teorema 4.5 temos:

Teorema 4.6 Seja $h(x, t)=h_{0}(x)+t g(x)$ uma deformação do germe $h_{0}$ com $\Gamma_{+}(g) \subset$ $C\left(\overline{I_{0}}\right)$ e $\Gamma_{+}\left(d g\left(\alpha_{i}\right)\right) \subset C\left(\overline{I_{0}}\right)$ entâo $\Gamma_{+}(g) \subset C(\bar{I})$ e, portanto $h_{t}$ é $C^{0}-\mathcal{R}_{V}$-trivial.

Prova: As hipóteses implicam em $g \in \overline{I_{0}}$ e $d g\left(\alpha_{i}\right) \in \overline{I_{0}}$. Então, pelo Lema 4.7, $g \in \bar{I}$ e portanto $\Gamma_{+}(g) \subset C(\bar{I})$. Pelo Teorema $4.2, h_{t}$ é $C^{0}-\mathcal{R}_{V^{-}}$trivial.

Corolário 4.1 Seja $I_{0}$ não degenerado e $h(x, t)=h_{0}(x)+t g(x)$ uma deformação do germe $h_{0}$ com $\Gamma_{+}(g) \subset \Gamma_{+}\left(I_{0}\right)$ e $\Gamma_{+}\left(d g\left(\alpha_{i}\right)\right) \subset \Gamma_{+}\left(I_{0}\right)$ então $\Gamma_{+}(g) \subset C(\bar{I})$ e, portanto $h_{t}$ é $C^{0}-\mathcal{R}_{V^{-t r i v i a l}}$. 
Prova: Do fato de $I_{0}$ ser não degenerado temos $\Gamma_{+}\left(I_{0}\right)=C\left(\overline{I_{0}}\right)$ e pelo teorema acima segue o resultado.

Exemplo 4.6 Seja $V \subseteq \mathbb{C}^{2}, 0$ definida por $\varphi(x, y)=x^{3}-y^{2}=0$, ou seja, $V$ é a cúspide. Temos que $\Theta_{V}$ é gerado por $\alpha_{1}=(2 x, 3 y), \alpha_{2}=\left(2 y, 3 x^{2}\right)$. Na $\mathcal{R}_{V}$ classificação dos germes $h: \mathbb{C}^{2} \rightarrow \mathbb{C}$ dado pelo Teorema 4.9 de [3] encontramos o germe $y^{2}+a x^{n}+t x^{n+1}$, $n \geq 4$ que é finitamente determinado para $a \neq 0$. Considere o germe $h_{0}(x, y)=y^{2}+a x^{n}$. Assim, $I_{0}=<2 a n x^{n}+6 y^{2}, 2 a n x^{n-1} y+6 x^{2} y>$, que é não degenerado. Portanto, $\left.C\left(\overline{T_{0}}\right)=\Gamma_{+}\left(I_{0}\right)\right)$. Então pelo Teorema $4.2 h_{t}$ é $C^{0}-\mathcal{R}_{V}$-trivial.

Exemplo 4.7 Seja $V \subseteq \mathbb{C}^{3}, 0$ parametrizada por $\left(x,-4 y^{3}-2 x y,-3 y^{4}-x y^{2}\right), V$ é o rabo de andorinha. Temos que $\Theta_{V}$ é gerado por $\eta_{1}=(2 x, 3 y, 4 z), \eta_{2}=\left(6 y,-2 x^{2}-\right.$ $8 z, x y)$ e $\eta_{3}=\left(-4 x^{2}-16 z,-8 x y, y^{2}\right)$. Na $\mathcal{R}_{V}$ classificação dos germes $h: \mathbb{C}^{3} \rightarrow \mathbb{C}$ dado pelo Teorema 4.10 de [3] encontramos o germe $z+a x^{n}+t x^{n+1}, n \geq 2$ que é finitamente determinado para $a \neq 0$ e para $n=2$ temos que ter também $a \neq 1 / 12$. Considere o germe $h_{0}(x, y, z)=z+a x^{n}$. Assim, $I_{0}=<2 a n x^{n}+4 z, 6 a n x^{n-1} y+$ $x y,-4 a n x^{n+1}-16 a n x^{n-1} z+y^{2}>$. Temos que $x^{n+1} \in \overline{d h_{t}\left(\Theta_{V}^{0}\right)}$. Então pelo Teorema $4.2 h_{t}$ é $C^{0}-\mathcal{R}_{V}$-trivial. 


\section{Capítulo 5}

\section{$C^{0}-\mathcal{K}_{R V^{-T}}$ Trivialidade Topológica}

Em [10], [12] T. Gaffney desenvolveu a noção de fecho integral de módulos, e obteve condiçōes suficientes para a trivialidade topológica de famílias de interseção completa com singularidade isolada em termos do fecho integral de um determinado módulo conveniente.

Neste capítulo, iniciamos o estudo da extensão dos resultados do capítulo anterior para o grupo $\mathcal{K}_{R V}$. Mais precisamente, o objetivo é obter condições para $C^{0}-\mathcal{K}_{R V^{-}}$ trivialidade de famílias de seções usando técnicas de fecho integral de módulos descrita por Gaffney.

Definição 5.1 Seja $X, x$ um germe de conjunto analítico complexo, e $M$ um submódulo de $\mathcal{O}_{X, x}^{p}$. Então $h \in \mathcal{O}_{X, x}^{p}$ pertence $\bar{M}$ se para toda curva $\varphi:(\mathbb{C}, 0) \rightarrow(X, x), h \circ \varphi$ pertence $a\left(\varphi^{*}(M)\right) \mathcal{O}_{1}$.

Seja $M$ um submódulo de $\mathcal{O}_{X, x}^{p}$ e $[M]$ a matriz dos geradores de $M, J_{k}(M)$ é o ideal gerado pelos menores $(k \times k)$ de $[M]$.

Lema 5.1 ([10], Lema 1.6) Suponha que $h \in \mathcal{O}_{X, x}^{p}, M \subset \mathcal{O}_{X, x}^{p}, J_{k+1}((h, M))=0$. Então

$$
J_{k}(M) . h \subseteq M . J_{k}((h, M))
$$

A conexão entre fecho integral de ideais e módulo é dada pela seguinte proposição:

Proposição 5.1 ([10], Proposição 1.7) Suponha que $h \in \mathcal{O}_{X, x}^{p}, M \subset \mathcal{O}_{X, x}^{p}$. Então $h \in \bar{M}$ se, e somente se, $J_{k}((h, M)) \subseteq \overline{J_{k}(M)}$ onde $k$ é o maior inteiro tal que $J_{k}((h, M)) \neq 0$. 
No que segue $\Gamma(E)$ denotará o conjunto das seções do fibrado vetorial $E$.

Proposição 5.2 ([10], Proposição 1.11) Suponha que $h \in \mathcal{O}_{X, x}^{p}, M \subset \mathcal{O}_{X, x}^{p}$. Então $h \in \bar{M}$ se, e somente se, para cada escolha de geradores de $s_{i}$ de $M$ existe uma vizinhança $U$ de $x$ tal que para todo $\varphi \in \Gamma\left(\operatorname{Hom}\left(\mathbb{C}^{p}, \mathbb{C}\right)\right),|\varphi(z) . h(z)| \leq C \sup _{i}\left|\varphi(z) . s_{i}(z)\right|$ para todo $z \in U$.

O resultado principal deste capítulo é o seguinte teorema:

Teorema 5.1 Seja $h_{0}:\left(\mathbb{C}^{n}, 0\right) \rightarrow\left(\mathbb{C}^{p}, 0\right) \mathcal{K}_{R V}$-finitamente determinado e $h:\left(\mathbb{C}^{n} \times\right.$ $\mathbb{C}, 0) \rightarrow\left(\mathbb{C}^{p}, 0\right)$ uma boa deformação analítica de $h_{0}$. Se $\frac{\partial h}{\partial t} \in \overline{d h_{t}\left(\Theta_{V}^{0}\right)+\mathcal{M}_{n} h^{*}\left(\mathcal{M}_{p}\right) \theta_{h_{t}}}$ então $h$ é $C^{0}-\mathcal{K}_{R V}$-trivial.

A demonstração do Teorema depende dos seguintes Lemas:

Lema 5.2 Se $J f\left(\Theta_{V}\right)+f^{*}\left(\mathcal{M}_{p}\right) \supseteq \mathcal{M}_{n}^{k}$ então $J f\left(\Theta_{V}\right)+\left(f^{*}\left(\mathcal{M}_{p}\right)\right)^{p} \supseteq \mathcal{M}_{n}^{r}$, ou seja, $\mathcal{V}\left(J f\left(\Theta_{V}\right)+\left(f^{*}\left(\mathcal{M}_{p}\right)\right)^{p}\right) \subseteq\{0\}$

Prova: Basta observar que $\mathcal{V}\left(J f\left(\Theta_{V}\right)+\left(f^{*}\left(\mathcal{M}_{p}\right)\right)^{p}\right)=\mathcal{V}\left(J f\left(\Theta_{V}\right)\right) \cap \mathcal{V}\left(\left(f^{*}\left(\mathcal{M}_{p}\right)\right)^{p}\right)=$ $\left.\mathcal{V}\left(J f\left(\Theta_{V}\right)\right) \cap \mathcal{V}\left(f^{*}\left(\mathcal{M}_{p}\right)\right)\right)=\mathcal{V}\left(J f\left(\Theta_{V}\right)+f^{*}\left(\mathcal{M}_{p}\right)\right) \supseteq\{0\}$ e o resultado segue.

Lema 5.3 Seja $h_{0}:\left(\mathbb{C}^{n}, 0\right) \rightarrow\left(\mathbb{C}^{p}, 0\right) \mathcal{K}_{R V}$-finitamente determinado e $h:\left(\mathbb{C}^{n} \times\right.$ $\mathbb{C}, 0) \rightarrow\left(\mathbb{C}^{p}, 0\right)$ uma deformação analítica de $h_{0}$. Pondo $h_{t}(x)=h(x, t)$, então para parâmetros suficientemente pequenos $h_{t}$ é $\mathcal{K}_{R V}$-finitamente determinado.

Dem: Pelo Lema 3.4 basta mostrarmos que $\left.\mathcal{V}\left(J h_{t} \Theta_{V}\right)\right) \cap h_{t}^{-1}(0) \subseteq\{0\}$.

Sejam $\Sigma=\left\{(x, t) \in \mathbb{C}^{\mathbf{n}} \times \mathbb{C}: x \in \mathcal{V}\left(J h_{t}\left(\Theta_{V}\right)\right)\right\} \mathrm{e}$

$$
\pi: \Sigma \cap h^{-1}(0) \rightarrow \mathbb{C}
$$

a projeção sobre o segundo fator. Então $\Sigma \cap h^{-1}(0)$ é uma variedade analítica e $(0,0) \in \pi^{-1}(0)$ é um ponto isolado por hipótese, assim $\pi$ é uma aplicação finita na vizinhança de $(0,0)$, ver [19] pag. $62-63$, e o resultado segue.

Passemos à demonstração do Teorema 5.1.

Prova: Sejam $\left\{\alpha_{1}, \ldots, \alpha_{m}\right\}$ geradores de $\Theta_{V}^{0}, M$ o submódulo gerado por $\left\{d h_{t}\left(\alpha_{1}\right), \ldots, d h_{t}\left(\alpha_{m}\right)\right\}, N$ o submódulo gerado por $\left\{x_{l} h_{t i} e_{j}, l=1, \ldots, n, i=1, \ldots, p ; j=\right.$ 
$1, \ldots, p\}$,onde $h_{t i}$ são as componentes de $h_{t},\left\{\rho_{1}, \ldots, \rho_{r}\right\}$ geradores de $J_{p}(M+N)$ e $\rho=\sum_{i=1}^{r}\left|\rho_{i}\right|^{2}$. Pelos Lemas 5.2 e 5.3 segue-se que $\rho_{t}(x)=0 \Leftrightarrow x=0$

Então pelo Lema 5.1

$$
\rho_{k} \cdot \frac{\partial h}{\partial t}=\sum_{j}^{m} d h_{t}\left(\alpha_{j}\right) a_{k j}+\sum_{l, i, j}^{n, p, p} x_{l} h_{t i} e_{j} b_{i j k}, \operatorname{com} a_{k j}, b_{i j k} \in J_{p}\left(\left(\frac{\partial h}{\partial t}, M+N\right)\right)
$$

assim,

$$
\left|\rho_{k}\right|^{2} \cdot \frac{\partial h}{\partial t}=\sum_{j}^{m} d h_{t}\left(\alpha_{j}\right) a_{k j} \overline{\rho_{k}}+\sum_{l, i, j}^{n, p, p} x_{l} h_{t i} e_{j} b_{i j k} \overline{\rho_{k}}
$$

segue que

$$
\rho . \frac{\partial h}{\partial t}=d h_{t}\left(\sum_{k, j}^{r, m} a_{k j} \overline{\rho_{k}} \alpha_{j}\right)+\sum_{l, i, j, k}^{n_{2}, p, p, r} \overline{\rho_{k}} b_{i j k} x_{l} e_{j} h_{t i}
$$

Por hipótese, e pela Proposição 5.1, temos que

$$
J_{p}\left(\left(\frac{\partial h}{\partial t}, M+N\right)\right) \subseteq \overline{J_{p}(M+N)}
$$

Então segue que $a_{k j}$ e $b_{i j k} \in \overline{J_{p}(M+N)}$

Seja $w(x, t)=\sum_{k, j}^{r, m} \frac{a_{k j} \overline{\rho_{k}} \alpha_{j}}{\rho}$. Por hipótese

$$
\left|a_{k j}\right| \leq A s u p_{i}\left\{\left|\rho_{i}\right|\right\}
$$

Assim, $|w(x, t)| \leq C|x|$, o que implica que $w$ é integrável. Seja

$$
\gamma(t, x, y)=\sum_{l, i, j, k}^{n, p, p, r} \frac{\left(\overline{\rho_{k}} b_{i j k} x_{l} e_{j}\right) y_{i}}{\rho}
$$

Por hipótese

$$
\left|b_{i j k}\right| \leq B s u p_{i}\left\{\left|\rho_{i}\right|\right\}
$$

Assim, $|\gamma(t, x, y)| \leq C|x||y|$, o que implica que $\gamma$ é integrável. Portanto $h$ é $C^{0}-\mathcal{K}_{R V^{-}}$ trivial.

Proposição 5.3 Se $h(x, t)=h_{0}(x)+t g(x)$ com $g \in \overline{d h_{0}\left(\Theta_{V}^{0}\right)+\mathcal{M}_{n} h_{0}^{*}\left(\mathcal{M}_{p}\right) \theta_{h_{0}}} e$ $\left(d g\left(\eta_{i}\right)+g_{j} e_{k} x_{u}\right) \in \overline{d h_{0}\left(\Theta_{V}^{0}\right)+\mathcal{M}_{n} h_{0}^{*}\left(\mathcal{M}_{p}\right) \theta_{h_{0}}}$, onde $\eta_{i}$ são geradores de $\Theta_{V}^{0}$ e $g_{j}$ as componentes de $g$ então $g \in \overline{d h_{t}\left(\Theta_{V}^{0}\right)+\mathcal{M}_{n} h_{t}^{*}\left(\mathcal{M}_{p}\right) \theta_{h_{t}}}$. 
Dem: Pela Proposição 5.2 temos que existe vizinhança $U$ de 0 em $\mathbb{C}^{p}$ tal que para todo $\phi \in \operatorname{Hom}\left(\mathbb{C}^{p}, \mathbb{C}\right)$

$$
|t|\left|\phi .\left(d g\left(\eta_{i}\right)+g_{j} e_{k} x_{u}\right)\right| \leq|t| \operatorname{csup}_{l, r, s, v}\left|\phi \cdot\left(d h_{0}\left(\eta_{l}\right)+h_{0_{r}} e_{s} x_{v}\right)\right|
$$

por outro lado,

$$
\begin{gathered}
\sup _{l, r, s, v}\left|\phi \cdot\left(d h_{t}\left(\eta_{l}\right)+h_{t_{r}} e_{s} x_{v}\right)\right|=\sup _{l, r, s, v}\left|\phi \cdot\left(d h_{0}\left(\eta_{l}\right)+h_{0_{r}} e_{s} x_{v}\right)+t \phi .\left(d g\left(\eta_{l}\right)+g_{r} e_{s} x_{v}\right)\right| \\
\geq \sup _{l, r, s, v}\left|\phi \cdot\left(d h_{0}\left(\eta_{l}\right)+h_{0_{r}} e_{s} x_{v}\right)\right|-|t| s u p_{l, r, s, v}\left|\phi .\left(d g\left(\eta_{l}\right)+g_{r} e_{s} x_{v}\right)\right| \\
\geq \sup _{l, r, s, v}\left|\phi \cdot\left(d h_{0}\left(\eta_{l}\right)+h_{0_{r}} e_{s} x_{v}\right)\right|-|t| \operatorname{csup}_{l, r, s, v}\left|\phi \cdot\left(d h_{0}\left(\eta_{l}\right)+h_{0_{r}} e_{s} x_{v}\right)\right| \\
\geq(1-\alpha) \sup _{l, r, s, v}\left|\phi .\left(d h_{0}\left(\eta_{l}\right)+h_{0_{r}} e_{s} x_{v}\right)\right|
\end{gathered}
$$

para algum $0<\alpha<1$ e $|t| \leq \frac{\alpha}{c}$. Desta desigualdade temos que

$$
\overline{d h_{0}\left(\Theta_{V}^{0}\right)+\mathcal{M}_{n} h_{0}^{*}\left(\overline{\mathcal{M}}_{p}\right) \theta_{h_{0}}} \subseteq \overline{d h_{t}\left(\Theta_{V}^{0}\right)+\mathcal{M}_{n} h_{t}^{*}\left(\mathcal{M}_{p}\right) \theta_{h_{t}}}
$$

donde segue o resultado.

Seria interessante obter resultado análogo ao Teorema 5.1 mas com a hipótese $\frac{\partial h}{\partial t} \epsilon$ $\overline{d h_{t}\left(\Theta_{V}^{0}\right)+\overline{h^{*}}\left(\mathcal{M}_{p}\right) \theta_{h_{t}}}$. Acreditamos que seja possível usar os métodos análogos aos de [31] e [32] para melhorar o resultado, e pretendemos dar continuidade a este estudo. 


\section{Capítulo 6}

\section{Equisingularidade de Famílias de Seções de Variedades Analíticas}

Neste capítulo vamos estudar o problema de equisingularidade para famílias de seções e mostrar que as hipóteses do Teorema 4.2 do capítulo 4 dão uma condição suficiente para esta equisingularidade.

\subsection{Os resultados de Teissier e Gaffney}

Bernard Teissier desenvolveu em [39] uma teoria infinitesimal e uma teoria de invariantes geométricos para estudar a equisingularidade de famílias de hipersuperfícies analíticas complexas $X_{t}^{d}$ com singularidades isoladas. $O$ fecho integral de um ideal $I$ é o objeto adequado para a parte infinitesimal desta teoria. Os invariantes geométricos são o número de Milnor de seções genéricas de $X_{t}$.

T. Gaffney estende os resultados de Teissier, usando métodos do fecho integral de módulos para obter condição necessárias e suficientes para a equisingularidade de famílias de interseções completas com singularidades isoladas.

Nessa seção vamos enunciar esses resultados. Começamos descrevendo o resultado de Teissier: Suponha que $F: \mathbb{C}^{n+1} \times \mathbb{C}, 0 \rightarrow \mathbb{C}$ define uma hipersuperfície com estrutura reduzida, $f_{t}(z)=F(z, t)$ define $X_{t}$, uma família de hipersuperfícies reduzidas de $\mathbb{C}^{n+1}$ com singularidade isolada na origem.

Sejam $\mu^{*}\left(X_{t}\right)$ a sequência de números de Milnor dada pelos números de Milnor $\mu^{k}$ das interseção de $X_{t}$ com um plano $k$-genérico, $\mu^{0}=1$ e $\mu^{n+1}\left(X_{t}\right)=\mu\left(X_{t}\right)$, o número 
de Milnor de $X_{t}$. Indiquemos ainda por $e(I)$ a multiplicidade de um ideal $I$.

Com estas condições Teissier obteve o seguinte:

Teorema 6.1 (Teissier, [40]) As seguintes condições são equivalentes:

(i) $X-T$ é diferenciável e Whitney regular sobre $T=\{0\} \times \mathbb{C}$

(ii) $\mu^{*}\left(X_{t}\right)$ é independente de $t$

(iii) $\frac{\partial F}{\partial t} \in \overline{\mathcal{M}_{n+1}\left(\frac{\partial F}{\partial z_{1}}, \ldots, \frac{\partial F}{\partial z_{n+1}}\right)}, X-T$ é diferenciável

(iv) $e\left(\mathcal{M}_{n+1} J\left(f_{t}\right)\right)$ em $\mathcal{O}_{X_{t}, 0}$ é constante.

A condição (iii) é conhecida como condição de $c$-equisingularidade de Teissier.

Como consequência do primeiro lema de isotopia vemos que as condições acima são condições suficientes para a trivialidade topológica da família $X_{t}$. Gaffney em [10], através da noção do fecho integral de módulos, obtém o objeto infinitesimal correto para estudar a Whitney equisingularidade de famílias de conjuntos analíticos. Um dos principais resultados obtidos por Gaffney em [10] é o seguinte teorema:

Teorema 6.2 Sejam $F: \mathbb{C}^{t} \times \mathbb{C}^{n}, 0 \rightarrow \mathbb{C}^{p}, 0$ holomorfa, com $X=F^{-1}(0)$ e $Y=$ $\mathbb{C}^{t} \times\{0\}$ e $X_{0}$ o conjunto dos pontos regulares de $X$. Então o par $\left(X_{0}, Y\right)$ é $W$-regular se, e somente se, $\frac{\partial F}{\partial s} \in \overline{\left\{x_{i} \frac{\partial F}{\partial x_{j}}\right\}}{ }_{\mathcal{O}_{X}}$ para todo vetor tangente $\frac{\partial}{\partial s}$ em $\{0\} \times \mathbb{C}^{t}$.

Neste capítulo procuramos mostrar que o estudo de seções de variedades analíticas admite uma formalização análoga a usada por Teissier e Gaffney, usando como objeto infinitesimal o fecho integral do espaço tangente ao grupo $\mathcal{R}_{V}$.

\section{2 $V$-Equisingularidade}

Seja $V \subset \mathbb{C}^{n}$ uma variedade analítica e $h: \mathbb{C}^{n} \times \mathbb{C}, 0 \rightarrow \mathbb{C}, 0, h(0, t)=0$, definindo uma família de seções de $V$ com $h$ uma boa deformação de um germe $\mathcal{R}_{V}$-finitamente determinado $h_{0}: \mathbb{C}^{n}, 0 \rightarrow \mathbb{C}, 0$.

Queremos definir equisingularidade para a família de seções de $V$, que chamaremos de $V$-equisingularidade da família $h$. Para tanto, vamos construir um diagrama de aplicações estratificadas que satisfaz ao segundo Lema de Isotopia de Thom.

De agora em diante, vamos assumir que em uma vizinhança $U$ da origem, $V$ admite uma estratificação de Whitney $\mathcal{S}_{V}$ para a qual $\{0\}$ é um estrato. Podemos ainda estender esta estratificação definindo uma estratificação $\mathcal{S}$ para a vizinhança $U$ de 0 
de forma natural, isto é, os estratos são os estratos de $\mathcal{S}_{V}$ e o complementar de $V$ em $U$. Denotemos por $\tilde{V}$ a subvariedade de $\mathbb{C}^{n} \times \mathbb{C}, 0$ definida por $\tilde{V}=V \times \mathbb{C}$. A estratificação produto é claramente uma estratificação Whitney regular para $\tilde{V} \mathrm{em}$ $U \times \mathbb{C}$ e esta pode também ser naturalmente estendida para uma estratificação $\tilde{\mathcal{S}}$ para $U \times \mathbb{C}$. Como $h: \mathbb{C}^{n} \times \mathbb{C}, 0 \rightarrow \mathbb{C}, 0$ é uma boa deformação de um germe $\mathcal{R}_{V}$-finitamente determinado $h_{0}$, podemos considerar $h: U \times B_{r}, 0 \rightarrow \mathbb{C}, 0$ onde $B_{r}$ é uma bola aberta em $\mathbb{C}$ contendo a origem.

Vamos agora refinar a estratificação $\tilde{\mathcal{S}}$ de $U \times B_{r}$ da seguinte forma: Dado um estrato $S$ de $\mathcal{S}$ definimos os novos estratos $\tilde{S}$ de $\tilde{\mathcal{S}}$ como um dos seguintes tipos: $\left(S \times B_{r}\right)-h^{-1}(0)$ e $\left(S \times B_{r}\right) \cap h^{-1}(0)$, onde $S$ é um estrato de $\mathcal{S}$. De fato este refinamento define uma nova estratificação para $U \times B_{r}$ já que por hipótese $h$ é transversal a $\tilde{V}$ fora do zero. Denotaremos também esta nova estratificação por $\tilde{\mathcal{S}}$. Seja $F: \mathbb{C}^{n} \times \mathbb{C}, 0 \rightarrow$ $\mathbb{C} \times \mathbb{C}, 0$ dada por $F(x, t)=(h(x, t), t)$.

Definição 6.1 Seja $f: \Omega \rightarrow \Sigma$ uma aplicação diferenciável. Uma estratificação de $f$ é um par $\left(\mathcal{S}, \mathcal{S}^{\prime}\right)$ com $\mathcal{S}$ e $\mathcal{S}^{\prime}$ estratificações de Whitney de $\Omega$ e $\Sigma$ respectivamente satisfazendo:

(i) $f$ aplica estrato em estrato;

(ii). Se $S \in \mathcal{S}$ é aplicado pela $f$ em $S^{\prime} \in \mathcal{S}^{\prime}$ então $f: S \rightarrow S^{\prime}$ é uma submersão.

Definição 6.2 Sejam $f: \Omega \rightarrow \Sigma$ uma aplicação diferenciável e $M, N \subseteq \Omega$ subvariedades tais que as restrições $f \mid M$ e $f \mid N$ têm rank constante e seja $x \in M$. Dizemos que $N$ é Thom regular (ou satisfaz a condição $A_{f}$ ) sobre $M$ em $x$ relativamente a $f$ quando: dada uma sequência $\left(y_{i}\right)$ em $N, y_{i} \rightarrow x$ tal que $\operatorname{ker} T_{y_{i}}(f \mid N) \rightarrow \tau$ então $\operatorname{ker} T_{x}(f \mid M) \subseteq \tau$. Dizemos que $N$ é Thom regular sobre $M$ relativamente a $f$ se $N$ é Thom regular sobre $M$ em todo ponto de $M$.

Definição 6.3 Sejam $\left(\mathcal{S}, \mathcal{S}^{\prime}\right)$ uma estratificação de $f: \Omega \rightarrow \Sigma$. Dizemos que $\left(\mathcal{S}, \mathcal{S}^{\prime}\right)$ é uma estratificação de Thom quando para quaisquer dois estratos $M, N \in \mathcal{S}$ temos $N$ Thom regular sobre $M$ relativamente a $f$.

Enunciamos a seguir o segundo lema de isotopia de Thom.

Teorema 6.3 (Segundo Lema de Isotopia de Thom, [18], [26]) Sejam $M \stackrel{G}{\rightarrow}$ $N \stackrel{\pi}{\rightarrow} P$ uma sequência de aplicações e variedades diferenciáveis. Sejam $A$ e $A^{\prime}$ subconjuntos fechados de $M$ e $N$ respectivamente que admitem estratificações de Whitney 
$\mathcal{S}$ e $\mathcal{S}^{\prime}$ respectivamente. Suponha que:

(i) $O \operatorname{par}\left(\mathcal{S}, \mathcal{S}^{\prime}\right)$ é uma estratificação de Thom para $G \mid A: A \rightarrow A^{\prime}$.

(ii) As restrições $G \mid A: A \rightarrow A^{\prime}$ e $\pi \mid A^{\prime}: A^{\prime} \rightarrow P$ são aplicações próprias.

(iii) A restrição $\pi \mid S^{\prime}: S^{\prime} \rightarrow P$ é uma submersão, para cada estrato $S^{\prime} \in \mathcal{S}^{\prime}$.

Então a aplicação estratificada $G: A \rightarrow A^{\prime}$ é localmente topologicamente trivial sobre $P$, isto é, para cada $y \in P$ existe uma vizinhança $U$ de y em $P$ e homeomorfismos $\alpha$ e $\beta$ preservando a estratificação natural, tal que o diagrama

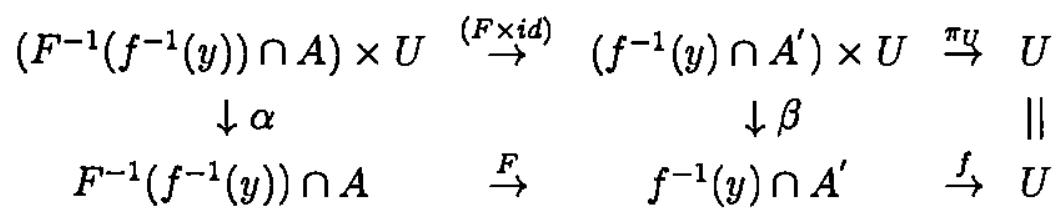

comuta.

Definição 6.4 Com a notação acima, $h$ é $V$-equisingular se existe $\varepsilon>0$ tais que: $(1)\left(B_{\varepsilon} \times B_{r}, \tilde{\mathcal{S}}\right)$ é Whitney regular;

(2) $B_{\varepsilon} \times B_{r} \stackrel{F}{\rightarrow} \mathbb{C} \times B_{r} \stackrel{\pi}{\rightarrow} B_{r}$ satisfaz o segundo Lema de Isotopia de Thom, onde $B_{\varepsilon}$ é a bola fechada em $\mathbb{C}^{n}$ de raio $\varepsilon$ e $B_{r}$ é a bola fechada em $\mathbb{C}$ de raio $r$, e $F: \mathbb{C}^{n} \times \mathbb{C}, 0 \rightarrow$ $\mathbb{C} \times \mathbb{C}, 0$ dada por $F(x, t)=(h(x, t), t)$.

Como consequência do segundo lema de isotopia temos que se $h$ é $V$-equisingular então $h$ é topologicamente $\mathcal{R}_{V}$-trivial.

$\mathrm{Na}$ sequência mostraremos que $\frac{\partial h}{\partial t} \in \overline{d h_{t}\left(\Theta_{V}^{0}\right)}$ é condição suficiente para a $V$ equisingularidade.

Teorema 6.4 Sejam $V=\phi^{-1}(0), \phi:\left(\mathbb{C}^{n}, 0\right) \rightarrow(\mathbb{C}, 0), h_{0}:\left(\mathbb{C}^{n}, 0\right) \rightarrow(\mathbb{C}, 0) \mathcal{R}_{V^{-}}$ finitamente determinado e $h:\left(\mathbb{C}^{n} \times \mathbb{C}, 0\right) \rightarrow(\mathbb{C}, 0)$ uma boa deformação de $h_{0}$. Suponhamos que $h^{-1}(0) \cap \Sigma_{\phi}=\{0\}$, onde $\Sigma_{\phi}$ é o conjunto singular de $\phi$ e $\frac{\partial h}{\partial t} \in \overline{\overline{d h_{t}}\left(\Theta_{V}^{0}\right)}$. Então h é $V$-equisingular.

J.W. Bruce em [5] considera uma questão análoga a proposta no Teorema acima. Ele descreve o tipo topológico de famílias genéricas de seções de uma variedade analítica, ou mais geralmente, seções de uma estratificação semialgébrica $\mathcal{T}$ de uma vizinhança da origem 0 em $\mathbb{R}^{n}$, sendo a origem um estrato. Tais famílias genéricas satisfazem à condição de transversal generalizada (G.T.) com relação à estratificação. Mais precisamente, dizemos que uma função $h: \mathbb{R}^{n} \rightarrow \mathbb{R}$ é transversal generalizada se 
para toda sequência $\left(x_{i}\right)$ em um estrato $S_{1}$ de $\mathcal{T}$ com $\lim _{i \rightarrow \infty} x_{i}=x \in S_{2}$ e o limite dos espaços tangentes $\lim _{i \rightarrow \infty} T_{x_{i}} S_{1}=T$ então $d h(x): T \rightarrow \mathbb{R}$ tem rank máximo, ou seja, $h^{-1}(h(x))$ é transversal a $T$.

O principal resultado de [5] é o seguinte teorema:

Teorema 6.5 Seja $\mathcal{T}$ uma estratificação de Whitney de uma vizinhança aberta $U$ da origem de $\mathbb{R}^{n}$, com a origem sendo um estrato. Seja $h: \mathbb{R}^{n} \times[0,1] \rightarrow \mathbb{R}$ uma familia de submersões, com $h(x, 0)=0$ e $h_{t}(x)=h(x, t)$. Suponha que a familia $h$ é transversal generalizada a $\mathcal{T}$, para todo $t \in[0,1]$. Então existe germe de homeomorfismo $G$ : $\left(\mathbb{R}^{n}, 0\right) \rightarrow\left(\mathbb{R}^{n}, 0\right)$ preservando estratos, tal que $h_{0} \circ G=h_{1}$.

Exemplos de famílias que satisfazem a condição de T.G. são seções de uma variedade analítica definidas por hiperplanos de $\mathbb{R}^{n}$. No nosso trabalho, a hipótese de G.T. é substituida pela condição de determinação finita e fecho integral. Desta forma, podemos obter a trivialidade topológica de famílias que não satisfazem à condição de G.T.

Exemplo 6.1 Seja $V \subseteq \mathbb{C}^{2}, 0$ definida por $\varphi(x, y)=x^{3}-y^{2}=0$. Temos que $\Theta_{V}$ é gerado por $\alpha_{1}=(2 x, 3 y), \alpha_{2}=\left(2 y, 3 x^{2}\right)$. Seja $h_{t}(x, y)=y+t x^{2}$ temos que $h_{t}$ é $\mathcal{R}_{V}$-finito e $x^{2} \in \overline{d h_{t}\left(\Theta_{V}^{0}\right)}$, mas $h_{t}$ não é transversal generalizada em 0 , pois $d h_{t}(0,0)=$ $(0,1)$ e o limite da reta tangente $T$ é o eixo-x.

\subsection{A Prova do Teorema}

Para a prova do Teorema 6.4 faremos uso do seguinte lema:

Lema 6.1 Sejam $h:\left(\mathbb{C}^{n+1}, 0\right) \rightarrow(\mathbb{C}, 0)$ e $G:\left(\mathbb{C}^{n+1}, 0\right) \rightarrow\left(\mathbb{C}^{2}, 0\right)$ dada por $G(x, t)=$ $(h(x, t), \phi(x))$. Se $g \in \overline{\left\langle d h\left(\eta_{i}\right)\right\rangle_{\mathcal{O}_{n+1}}}$ então $(g, 0) \in \overline{\left\langle x_{i} \frac{\partial G}{\partial x_{j}}\right\rangle_{\mathcal{O}_{G^{-1}(0)}}}$, onde $\eta_{i}$ são geradores de $\Theta_{V}^{0}$.

Prova: Por hipótese, para toda $\varphi:(\mathbb{C}, 0) \rightarrow\left(\mathbb{C}^{n+1}, 0\right), g \circ \varphi \in<d h\left(\eta_{i}\right) \circ \varphi>$. Então para toda $\varphi:(\mathbf{C}, 0) \rightarrow(V \times \mathbb{C}, 0)$, temos a seguinte condição $(g \circ \varphi, 0) \in<$ $d h\left(\eta_{i}\right) \circ \varphi, d \phi\left(\eta_{i}\right) \circ \varphi>$ pois $d \phi\left(\eta_{i}\right) \in<\phi>, \operatorname{Im}(\varphi) \subset V \times \mathbb{C}$ e $\phi(V)=0$.

Portanto $(g \circ \varphi, 0) \in<\left(x_{i} \frac{\partial h}{\partial x_{j}}, x_{i} \frac{\partial \phi}{\partial x_{j}}\right) \circ \varphi>$. Logo, $(g, 0) \in \overline{\left\langle\left(x_{i} \frac{\partial h}{\partial x_{j}}, x_{i} \frac{\partial \phi}{\partial x_{j}}\right)\right\rangle}=$

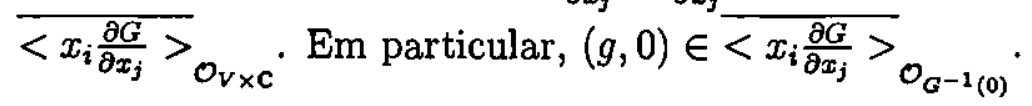


Observação 6.1 Observemos que o resultado permanece verdadeiro com a hipótese

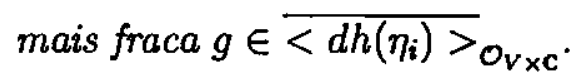

O exemplo abaixo mostra que a hipótese de pertencer ao fecho integral de $<d h\left(\eta_{i}\right)>_{O_{V \times \mathbf{c}}}$ é mais forte que a condição de pertencer ao fecho integral de $<x_{i} \frac{\partial G}{\partial x_{j}}>_{O_{G^{-1}(0)}}$.

Exemplo 6.2 Sejam $\phi:\left(\mathbb{C}^{3}, 0\right) \rightarrow(\mathbb{C}, 0)$ dada por $\phi(x, y, z)=2 x^{2} y^{2}+y^{3}-z^{2}+x^{4} y$ e $\phi^{-1}(0)=V, h:\left(\mathbb{C}^{4}, 0\right) \rightarrow(\mathbb{C}, 0)$ dada por $h(x, y, z, t)=y+(a+t) x^{2}$ e $G:\left(\mathbb{C}^{4}, 0\right) \rightarrow$ $\left(\mathbf{C}^{2}, 0\right)$ dada por $G(x, y, z, t)=\left(y+(a+t) x^{2}, 2 x^{2} y^{2}+y^{3}-z^{2}+x^{4} y\right)$. Temos que $\Theta_{V}$ é gerado por $\eta_{1}=(2 x, 4 y, 6 z), \eta_{2}=\left(0,2 z, x^{4}+4 x^{2} y+3 y^{2}\right), \eta_{3}=\left(x^{2}+3 y,-4 x y, 0\right), \eta_{4}=$ $\left(z, 0,2 x^{3} y+2 x y^{2}\right)$. Como vimos anteriormente, no Exemplo $4.4, x^{2} \notin \overline{\left\langle d h\left(\eta_{i}\right)\right\rangle_{\mathcal{O}_{4}}}$

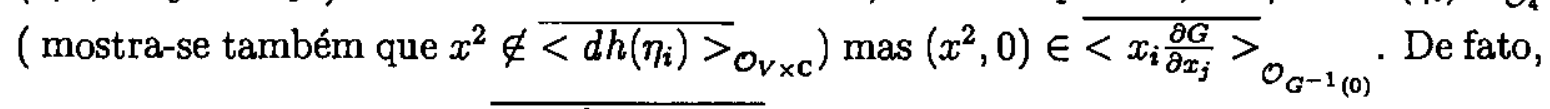
mostraremos que $\left(x^{2}, 0\right) \in \overline{\left\langle x_{i} \frac{\partial G}{\partial x_{j}}, e_{i} G_{j}\right\rangle_{O_{4}}}$ o que implicará no resultado. Temos que

$$
\begin{aligned}
& \text { (a) } z G_{z}=\left(0,-2 z^{2}\right) \\
& \text { (b) } e_{1} G_{1}=\left(y+(a+t) x^{2}, 0\right) \\
& \text { (c) } x^{2} G_{y}=\left(x^{2}, 4 x^{4} y+3 x^{2} y^{2}+x^{6}\right) \\
& \text { (d) } e_{2} G_{2}+\frac{1}{2} z G_{z}=\left(0,2 x^{2} y^{2}+y^{3}+x^{4} y\right)
\end{aligned}
$$

Seja $\varphi:(\mathbb{C}, 0) \rightarrow\left(\mathbb{C}^{4}, 0\right)$ dada por $\varphi(u)=\left(\varphi_{1}(u), \varphi_{2}(u), \varphi_{3}(u), \varphi_{4}(u)\right)$. Verifiquemos que $\left(\varphi_{1}^{2}, 0\right) \in<\left(x_{i} \frac{\partial G}{\partial x_{j}}, e_{i} G_{j}\right) \circ \varphi>_{\mathcal{O}_{1}}$. Sejam $r=\operatorname{ord}\left(\varphi_{1}\right)$ e $s=\operatorname{ord}\left(\varphi_{2}\right)$ se $s \leq r$ ou $2 s=r$ então por $(b)\left(\varphi_{1}^{2}, 0\right) \in<\left(x_{i} \frac{\partial G}{\partial x_{j}}, e_{i} G_{j}\right) \circ \varphi>\mathcal{O}_{1}$.

Se $s>r$ então de $(c)$

$$
x^{2} G_{y} \circ \varphi=\left(\varphi_{1}^{2}, 4 \varphi_{1}^{4} \varphi_{2}+3 \varphi_{1}^{2} \varphi_{2}^{2}+\varphi_{1}^{6}\right)=\left(\varphi_{1}^{2}, 0\right)+\left(0,4 \varphi_{1}^{4} \varphi_{2}+3 \varphi_{1}^{2} \varphi_{2}^{2}+\varphi_{1}^{6}\right)
$$

e de $(d)$ temos que $\left(0,4 \varphi_{1}^{4} \varphi_{2}+3 \varphi_{1}^{2} \varphi_{2}^{2}+\varphi_{1}^{6}\right) \in<\left(x_{i} \frac{\partial G}{\partial x_{j}}, e_{i} G_{j}\right) \circ \varphi>_{\mathcal{O}_{1}}$, portanto, $\left(\varphi_{1}^{2}, 0\right) \in<\left(x_{i} \frac{\partial G}{\partial x_{j}}, e_{i} G_{j}\right) \circ \varphi>_{\mathcal{O}_{1}}$, ou seja, $\left(x^{2}, 0\right) \in \overline{\left\langle x_{i} \frac{\partial G}{\partial x_{j}}, e_{i} G_{j}\right\rangle_{\mathcal{O}_{4}}}$.

Passamos à demonstração do Teorema 6.4, ela é análoga a dada por Bruce em [5].

Dividiremos a demonstração em várias afirmações.

Afirmação 1. A estratificação $\tilde{S}$ é Whitney regular.

Prova: A Whitney regularidade de um par de estratos $\left(S_{1}, S_{2}\right)$ é imediata, com exceção da regularidade sobre $\{0\} \times \mathbb{C}$. Mas claramente os estratos da forma 
$(S \times \mathbb{C})-h^{-1}(0)$ são regulares sobre $\{0\} \times B_{r}$ uma vez que a estratificação original é regular. Resta provar que $\left(S \times B_{r}\right) \cap h^{-1}(0)$ é regular sobre $\{0\} \times B_{r}$. Da hipótese $\frac{\partial h}{\partial t} \in \overline{d h_{t} \bar{\Theta}_{V}^{0}}$ segue que $\left(\frac{\partial h}{\partial t}, 0\right) \in \overline{\left\langle x_{i} \frac{\partial G}{\partial x_{j}}\right\rangle_{\mathcal{O}_{G^{-1}(0)}}}$ (Lema 6.1) e pelo Teorema $6.2,\left(G^{-1}(0)-\Sigma_{G^{-1}(0)},\{0\} \times B_{r}\right)=\left(h^{-1}(0) \cap \tilde{V}-\{0\} \times B_{r},\{0\} \times B_{r}\right)$ é W. regular. $\diamond$

Afirmação 2. Para algum $\varepsilon^{\prime}>0$ e todo $0<\varepsilon \leq \varepsilon^{\prime}$ o produto da fronteira da $\varepsilon$-bola, $\partial B_{\varepsilon}$, com $B_{r}$ encontra os estratos de $\tilde{\mathcal{S}}$ transversalmente.

Prova: Suponhamos que a afirmação seja falsa. Então podemos encontrar uma sequência $\left(x_{i}, t_{i}\right)$ em algum estrato $\tilde{S}$ com $x_{i} \rightarrow 0$ e $T_{\left(x_{i}, t_{i}\right)} \tilde{S} \subset T_{\left(x_{i}, t_{i}\right)}\left(\partial B_{\varepsilon_{i}} \times B_{r}\right)$ onde $\varepsilon_{i}=\left\|x_{i}\right\|$. Então $\left(x_{i}, 0\right)$ é perpendicular a $T_{\left(x_{i}, t_{i}\right)} \tilde{S}$. Isto contradiz a condição B de Whitney.

Temos uma primeira aproximação para um diagrama estratificado, isto é,

$$
B_{\varepsilon} \times B_{r} \stackrel{F}{\rightarrow} \mathbb{C} \times B_{r} \stackrel{\pi}{\rightarrow} B_{r}
$$

onde $B_{\varepsilon}$ é a bola fechada em $\mathbb{C}^{n}$ de raio $\varepsilon \operatorname{com} \varepsilon \leq \varepsilon^{\prime}, F(x, t)=(h(x, t), t)$ e $\pi$ a projeção no segundo fator. Estratificamos $\mathbb{C} \times B_{r}$ por $(\mathbb{C}-\{0\}) \times B_{r} \cup\{0\} \times B_{r}$ e refinamos a estratificação de $B_{\varepsilon} \times B_{r}$, tomando a interseção dos estratos de $\tilde{\mathcal{S}}$ com $\partial B_{\varepsilon} \times B_{r}$ e $i n t B_{\varepsilon} \times B_{r}$.

Gostariamos de provar que esta estratificação satisfaz à condição de Thom, mas $h_{t}$ poderá ter pontos críticos sobre $\partial B_{\varepsilon}$. Para contornar este problema necessitamos do seguinte.

Afirmação 3. Para algum $\delta>0, B_{\delta}-\{0\}$ em $\mathbb{C}$ consiste somente de valores regulares de $h_{t}$ para todo $t \in B_{r}$.

Prova: Segue do Teorema 3.4 e do fato de $h$ ser uma boa deformação

No diagrama acima trocamos $\mathbb{C}$ por $B_{\delta}$, onde $B_{\delta}$ é a bola de raio $\delta$, estratificada por $\partial B_{\delta} \cup\{0\} \cup i n t B_{\delta}-\{0\}$, satisfazendo as condições da afirmação 3. Obtemos assim uma nova estratificação $F^{-1}\left(B_{\delta} \times B_{r}\right)$ "pulling back" os estratos. Consideremos agora

$$
F^{-1}\left(B_{\delta} \times B_{r}\right) \stackrel{F}{\rightarrow} B_{\delta} \times B_{r} \stackrel{\pi}{\rightarrow} B_{r}
$$

Afirmação 4. O diagrama acima é Thom estratificado. 
Prova: Que as estratificações são Whitney regulares e estratos são aplicados submersivamente em estratos é trivial. Assim resta somente mostrar a condição de Thom regularidade ou $A_{h_{t}}$. Dados dois estratos $\tilde{S}_{1}, \tilde{S}_{2} \operatorname{com}\left(x_{i}, t_{i}\right) \in \tilde{S}_{1}$, e $\left(x_{i}, t_{i}\right) \rightarrow(x, t) \in$ $\tilde{S}_{2}$, o núcleo de $d F\left(x_{i}, t_{i}\right)$ restrito a $T_{\left(x_{i}, t_{i}\right)} \tilde{S}_{1}, K_{i}$, é $T_{\left(x_{i}, t_{i}\right)} \tilde{S}_{1} \cap\left(\operatorname{ker} d h_{t_{i}}\left(x_{i}\right) \times\{0\}\right)$. O limite destes núcleos é $T \cap\left(\operatorname{ker} d h_{t}(x) \times\{0\}\right)$ onde $T=\lim _{i \rightarrow \infty} T_{\left(x_{i}, t_{i}\right)} \tilde{S}_{1}$. Se $x \neq 0$ então ker $d h_{t}(x) \times\{0\}$ é transversal a $T$, portanto $\lim _{i \rightarrow \infty} K_{i}=T \cap\left(\operatorname{ker} d h_{t}(x) \times\{0\}\right)$. Como $T \supset T_{(x, t)} \tilde{S}_{2}$ (condição A de Whitney), logo $\lim _{i \rightarrow \infty} K_{i}$ contém o núcleo de $d F(x, t)$ restrito a $T_{(x, t)} \tilde{S}_{2}$. Se $x=0$ então $\tilde{S}_{2}=\{0\} \times B_{r}$, neste caso a Thom regularidade é trivial.

\subsection{Observação final}

Além da condição $A_{f}$ de Thom definida acima, uma outra condição associada à equisingularidade de família de funções foi introduzida por J. Henry, M. Merle e C. Sabbah em [21] que é a condição $W_{f}$. T. Gaffney e S.Kleiman em trabalhos recentes ([13], [14], [22]) têm estudado o problema da equisingularidade de família de interseções completas com sigularidades isoladas, bem como o de famílias de funções definidas em variedades analíticas.

Em especial, em [13] T.Gaffney e S.Kleiman relacionam a condição $W_{f}$ com o fecho integral de um conveniente módulo. Mais precisamente, seja $(X, 0)$ um germe de variedade analítica equidimensional em $\left(\mathbb{C}^{n}, 0\right),(Y, 0)$ um subgerme. Seja $f:\left(\mathbb{C}^{n}, 0\right) \rightarrow\left(\mathbb{C}^{q}, 0\right)$ um germe de aplicação cuja restrição $f \mid Y$ é uma submersão sobre um subgerme analítico fechado de $\mathbb{C}^{\mathbb{q}}$. Assuma que exista um subconjunto analítico aberto e denso $X_{0}$ de $X$ tal que $f \mid\left(X_{0}, 0\right)$ é uma submersão sobre sua imagem e tem fibras equidimensionais.

Definição 6.5 O par $\left(X_{0}, Y\right)$ satisfaz à condição $W_{f}$ em 0 se existe vizinhança euclidiana $U$ de 0 em $X$ e constante $C>0$ tais que, para todo $y \in U \cap Y$ e $x \in U \cap X_{0}$, temos

$$
\operatorname{dist}\left(T_{y} Y(f(y)), T_{x} X(f(x))\right) \leq C \operatorname{dist}(x, Y)
$$

onde $T_{y} Y(f(y))$ e $T_{x} X(f(x))$ são os espaços tangentes das fibras indicadas das retrições $f \mid Y$ e $f \mid X$ (são espaços tangentes a $f^{-1}(y) \cap Y$ e $\left.f^{-1}(x) \cap X\right)$. 
Assim, a condição $W_{f}$ nos diz que hiperplanos tangentes as fibras de $f$ em $X_{0}$ se aproximam de $T_{y} Y(f(y))$ mais rápido do que $x$ se aproximam de $Y$. Quando $f$ é uma aplicação constante a condição $W_{f}$ se reduz a condição $W$. Também, quando $X$ é $\mathbb{C}^{n}$ e $f$ é uma função, $W_{f}$ se reduz a "c-equisingularidade" de Teissier [38], [39]. A importância de estudar $W_{f}$ consiste no seguinte fato: Se $(X-Y, Y)$ é uma estratificação satisfazendo $W_{f}$, então o par $X, f$ é localmente topológicamente $\mathcal{R}$-trivial sobre $Y$, ou seja, existe homeomorfismo $h: X(0) \times Y \rightarrow X$ tal que $f \circ h=(f \mid X(0)) \times I_{Y}$, onde $X(0)$ é a fibra de uma retração $r: X \rightarrow Y$ e $I_{Y}$ é a identidade de $Y$.

Seja $(X, 0)$ definido pelo anulamento de $F:\left(\mathbb{C}^{n}, 0\right) \rightarrow\left(\mathbb{C}^{p}, 0\right)$. Denotando por $J M(F ; f)$ o módulo jacobiano gerado pelas colunas da matriz jacobiana de $(F, f)$, isto é, o módulo gerado por $\left\langle\left(x_{i} \frac{\partial F}{\partial x_{j}}, x_{i} \frac{\partial f}{\partial x_{j}}\right)\right\rangle$. Pondo $\mathbb{C}^{n}=\mathbb{C}^{l} \times Y$, sejam $J M(F ; f)_{l}$ e $J M(F ; f)_{n-l}$ os submódulos de $J M(F ; f)$ gerado pelas derivadas parciais com respeito as $l$ primeiras variáveis e com respeito as últimas $n-l$ variáveis de $\mathbb{C}^{n}$, respectivamente. Finalmente, seja $\mathcal{M}_{Y}$ o ideal de $Y$ em $\mathbb{C}^{n}$, ou seja, o ideal gerado pelas $l$ primeiras variáveis de $\mathbb{C}^{n}$.

Proposição 6.1 (Proposição (1.1), [14])Com as notações acima, as seguintes condições são equivalentes:

(i) o par $\left(X_{0}, Y\right)$ satisfaz $W_{f}$ em 0 ;

(ii) $J M(F ; f)_{n-l} \subseteq \overline{\mathcal{M}_{Y} J M(F ; f)_{l}}$;

Observamos que a proposição acima é uma generalização do Teorema 6.2 , basta tomar $f$ constante.

$\mathrm{O}$ nosso teorema sobre $V$-equisingularidade, Teorema 6.4, foi obtido independentemente do resultado acima de T. Gaffney e S. Kleiman. Entretanto, observamos que tomando $X$ do teorema acima como $\tilde{V}=V \times \mathbb{C}$ e fazendo $f=h$, temos que a condição $\frac{\partial h}{\partial t} \in \overline{d h\left(\Theta_{V}^{0}\right)}$ implica em $\left(0, \frac{\partial h}{\partial t}\right) \in \overline{\mathcal{M}_{Y}<\left(\frac{\partial \phi}{\partial x_{i}}, \frac{\partial h}{\partial x_{i}}\right)>}=\overline{\mathcal{M}_{Y} J M(\phi ; f)_{l}}$, onde $\phi$ é a equação de definnição de $V$.

Portanto, o seguinte resultado é consequência deste fato e do Teorema 6.1 acima:

Corolário 6.1 Com as notações acima e tomando-se $Y=\mathbb{C}$, se $\frac{\partial h}{\partial t} \in \overline{d h\left(\Theta_{V}^{0}\right)}$ então o $\operatorname{par}(\tilde{V}-\mathbb{C}, \mathbb{C})$ satisfaz $W_{h}$ em 0 . 


\section{Referências Bibliográficas}

[1] Atique, R. W., Classificação e topologia de variedades singulares parametrizadas por multigermes, Tese de Doutorado, Instituto de Ciências Matemáticas e de Computação, USP-São Carlos, 1998.

[2] Chang, S.H. and Lu, Y.C., On $C^{0}$-sufficiency of complex jets, Canad. J. Math. 25(1973), 874-880.

[3] Bruce, J.W., Kirk, N.P. and du Plessis, A.A., Complete transversals and the classification of singularities, Nonlinearity 10 (1997), 253-275.

[4] Bruce, J.W. and Roberts, R.M., Critical points of functions on analytic varieties, Topology 27 (1988), 57-90.

[5] Bruce, J.W., Generic functions on semialgebraic sets, Quart. J. Math. Oxford (2), 37 (1986), 137-165.

[6] Damon, J., Deformations of sections of singularities and Gorenstein surface singularities, Amer. J. Math., 109 (1987), 695-722.

[7] Damon, J., A-equivalence and the equivalence of sections of images and discriminants, Singularity Theory and its Applications: Warwick 1989, Part I, Lecture Notes in Math., 1462(1991), 93-121, Springer-Verlag, New York.

[8] Dimca, A., Function germs defined on isolated hypersurface singularities, Composition Math. 53 (1984),245-258.

[9] Fuster, M.C.R., Mancini, S. and Ruas, M.A.S., Stability and genericity of composition of maps, Matemática Contemporânea, 5 (1993), 29-51. 
[10] Gaffney, T., Integral closure of modules and Whitney equisingularity, Inventiones Mathematicae, 107(1992), 301-322.

[11] Gaffney, T., Properties of finitely determined germs, Thesis, Brandeis University, 1975.

[12] Gaffney, T., Multiplicities and equisingularity of ICIS germs, Inventiones Mathematicae, 123(1996), 209-220.

[13] Gaffney, T. and Kleiman, S.L. Specialization of integral dependence for modules, preprint.

[14] Gaffney, T. and Kleiman, S.L. $W_{f}$ and integral dependence, preprint.

[15] Gaffney, T. and Wilson, L., Equivalence of generic mappings and $C^{\infty}$ normalization, Compositio Mathematica 49(1983), 291-308.

[16] Gaffney, T. and Wilson, L., Equivalence theorems in global singularity theory, Proc. of Symposia in Pure Math.40(1983), 439-447.

[17] Gibson,C.G., Singular points of smooth mappings. Research Notes in Mathematics, 25. London, Pitman.

[18] Gibson,C.G., Wirthmüller, K., Du Plessis, A.A. and Looijenga, E.J.N., Topological stability of smooth mappings, Lecture Notes in Math. 552(1976), SpringerVerlag, New York.

[19] Grauert, H. and Remmert, R., Coherent Analytic Sheaves, Springer-Verlag, New York (1984).

[20] Gunning, R. C. and Rossi,H., Analytic functions of several complex variables, Prentice-Hall, 1965.

[21] Henry, J.P.G., Merle, M. and Sabbah, C., Sur la condition de Thom stricte pour un morphime analytique complexe, Ann. Scient. Éc. Norm. Sup. 17(1984), 227-268.

[22] Kleiman, S.L., Equisingularity, multiplicity, and dependence,preprint. 
[23] Kouchnirenko, A. G., Polyèdres de Newton et nombres de Milnor, Inventiones Mathematicae, 32(1976), 1-31.

[24] Kuo,T. C., On $C^{0}$-sufficiency of jets of potential functions, Topology, 8(1969), 167-171.

[25] Kuo,T. C., The ratio test for analytic Whitney stratifications, Liverpool Singularities Symposium, Lecture Notes in Maths 192(1971).

[26] Marar,W.L., Mapping fibrations and multiple point schemes, Thesis, University of Warwick, 1989.

[27] Mather, J.N., Stability of $C^{\infty}$-mappings IV: Classification of stable map-germs by R-algebras, Publ. Math. IHES 37 (1970), 223-248.

[28] Mond, D., On the classification of maps from $\mathbb{R}^{2}$ to $\mathbb{R}^{3}$, Proc. London Math. Soc., 50 (1985), 333-369.

[29] Pellikaan, R., Finite determinacy of functions with non-isolated singularities, Proc. London Math. Soc., (3) 57 (1988), 357-382.

[30] Izumiya, S. and Matsuoka, S., Notes on smooth functions germs on varieties, Proc. Amer. Math. Soc. 97 (1989), 146-150.

[31] Ruas, M.A.S., On the degree of $C^{l}$-determinacy, Math. Scand., 59 (1986), 59-70.

[32] Ruas, M.A.S. and Saia, M.J., $C^{l}$-determinacy of weighted homogeneous germs, Hokkaido Mathematical Journal, 26 (1997), 89-99.

[33] Ruas, M.A.S. and Saia, M.J., The polyhedron of equisingularity of germs of hipersurfaces, Pitman Resarch Notes in Mathematics Series, "Proceedings of 3rd Real and Complex Singularities Congress", S. Carlos - USP, Brasil 333(1995), $37-48$.

[34] Rieger, J.H., Families of maps from the plane to the plane, J. London Math. Soc. 36, 2, 351-369 (1987).

[35] Saia, M., The integral closure of ideals and the Newton filtration, J. Algebraic Geometry, 5(1996), 1-11. 
[36] Saia, M., The integral closure of ideals and Whitney equisingularity of gerims of hypersurfaces, Matemática Contemporânea, 12 (1997), 183-198.

[37] Saito, K., em Theory of logarithmic differential forms and, logarithmic vart fields, J. Fac. Sci. Univ. Tokyo Sect. 1A Math. 27 (1980), 265-291.

[38] Teissier, B., Introduction to Equisingularity Problems, Proceedings of Symposia in Pure Mathematics 29 (1977), 593-631.

[39] Teissier, B., Multiplicities polaires, sections planes, et conditions de Whitney, Lecture Notes in Math 961 (1982), 314-491.

[40] Teissier, B., Cycles évanescents, sections planes et conditions de Whitney, Astérisque 7-8 (1973), 285-362.

[41] Trotman, D., Comparing regularity conditions on stratifications, Proceedings of Symposia in Pure Mathematics 40 (1983), 575-586.

[42] Trotman, D. and Wilson,L.C., Stratifications and finite determinacy, Proc. London Math. Soc. (3) 78 (1999), 334-368.

[43] Wall, C.T.C., Finite determinacy of smooth map-germs, Bull. London Math. Soc. 13(1981), 481-539.

[44] West, J. M., The differential geometry of the crosscap, Ph.D. Thesis, University of Liverpool, 1995.

[45] Whitney, H., Tangents to an analytic variety, Ann. of Math. 81(1965),496-549.

[46] Wilson, L.C., Stratifications and sufficiency of jets, Singularity theory (Triestf 1991), 953-973, World Sci. Publishing, River Edge, NJ, 1995.

[47] Yoshinaga, E., Topologically principal part of analytic functions, Transactions the American Math. Soc. 314 (1989), 803-814. 